\title{
Processos com Memória \\ Longa Compartilhada
}

\author{
João Ricardo Sato
}

\author{
DISSERTAÇÃO APRESENTADA \\ $\mathrm{AO}$ \\ INSTITUTO DE MATEMÁTICA E ESTATÍSTICA \\ DA \\ UNIVERSIDADE DE SÃO PAULO \\ PARA \\ OBTENÇÃO DO GRAU DE MESTRE \\ EM \\ ESTATÍSTICA \\ Área de Concentração: Estatística \\ Orientador: Prof. Dr. Pedro Alberto Morettin
}

Durante o desenvolvimento deste trabalho, o autor recebeu apoio financeiro do $\mathrm{CNPq}$

— São Paulo, Junho de 2004 — 


\title{
Processos com Memória \\ Longa Compartilhada
}

\author{
Este exemplar corresponde à redação \\ final da dissertação devidamente corrigida \\ e apresentada por João Ricardo Sato \\ e aprovada pela comissão julgadora.
}

São Paulo, 29 de junho de 2004.

Banca examinadora:

- Prof. Dr. Pedro Alberto Morettin (orientador) (IME-USP)

- Prof. Dr. Chang Chiann (IME-USP)

- Prof. Dr. Denisard Cneio de Oliveira Alves (FEA-USP) 


\section{Resumo}

Este trabalho tem como objetivo a avaliação de três estimadores do parâmetro de integração fracionária $d$ e de um teste para memória longa compartilhada. Os estimadores a serem avaliados são: o estimador de Geweke e Porter-Hudak, o estimador usando o periodograma suavizado e o estimador semiparamétrico truncado de Whittle. A avaliação dos estimadores será no contexto de processos ARFIMA+ARMA, e em relação a variações nos termos autoregressivos e de médias móveis, tanto do termo de memória curta quanto do termo de memória longa. Além disso, serão introduzidos o conceito de modelos com memória longa compartilhada e um método de identificação através da análise de correlação canônica para séries temporais multivariadas proposto, por Ray e Tsay (1997). Por fim, serão apresentadas três aplicações sobre dados reais dos tópicos estudados: uma para a velocidade do vento em São Paulo e Piracicaba e outras duas para séries das bolsas de valores de Hong Kong, Nova Zelândia, Singapura, Brasil e Reino Unido. 


\begin{abstract}
The goal of this project is the evaluation of three long memory parameter estimators and a common long range dependence test. The estimators evaluated are: the Geweke and Porter-Hudak, the smoothed periodogram and the semiparametric truncated Whittle estimators. The evaluation is in the context of processes ARFIMA+ARMA, and related to variations in the autoregressive and moving average coefficients, both in the short and long memory terms. Furthermore, we describe common long range dependence processes and an identification approach (Ray and Tsay, 1997) for them, using the canonical correlation analysis. Finally, three applications to real data are presented: the first one to the wind's speed in the Brazilian cities of São Paulo and Piracicaba, and the other ones to financial time series of the stock markets of Hong Kong, New Zealand, Singapore, Brazil and the United Kingdom.
\end{abstract}


Primeiramente, agradeço a Deus por todas as oportunidades oferecidas. Gostaria de agradecer principalmente aos meus pais, pela formação e apoio incondicional dados. Um especial agradecimento ao Prof. Pedro Alberto Morettin, que desde meu segundo ano de graduação se disponibilizou a ensinar-me, e pelo exemplo de competência e caráter. Agradeço também aos meus irmãos Cristiane, Rafael, Paula, Priscila e a todos os familiares por estarem presentes em minha vida, e a Cristina pelo carinho e apoio. Aos meus amigos Fábio, Luis Gustavo, Ricardo, Igor, Renatinha, Carlos, Marcus, Noemi, Ronaldo, Regina, Ayumi, Seiko Takano, Márcio Masaki, Tatyana, Carlão, André, Marcelo, Paulo, Márcio Medeiros, Mayra, Cleber, Marcos, Juvêncio, Miriam, Gladys, Fred, Elias, Sueli, Juan, Kelly, Sumaya, Célia, Cristiane, Andre, Karina e Allan. Finalmente, meus sinceros agradecimentos aos professores Carlos Bragança Pereira, Chang Chiann, Mariane Streibel, Clélia Toloi e aos demais professores do Departamento de Estatística do IME-USP. 


\section{Conteúdo}

$\begin{array}{ll}\text { Introdução } & 1\end{array}$

1 Conceitos e Definições 4

2 Modelos Clássicos de Séries Temporais $\quad 9$

2.1 Processo Linear . . . . . . . . . . . . . . . . . . 9

2.2 Modelos da família ARIMA $(\mathrm{p}, \mathrm{d}, \mathrm{q}) \ldots \ldots \ldots \ldots$

2.2.1 Modelo Autoregressivo . . . . . . . . . . . . . . . . . . 11

2.2.2 Modelo de Médias Móveis . . . . . . . . . . . . . . . . . 12

2.2.3 Modelo Autoregressivo e de Médias Móveis . . . . . . . . . . . . . . 13

2.2.4 Modelo Autoregressivo e de Médias Móveis Integrado . . . . . . . 14

2.3 Modelos Heterocedásticos Condicionais . . . . . . . . . . . . . . . . . . 15

2.3.1 Modelos da Família GARCH . . . . . . . . . . . . . . . . . 17

2.3.2 Modelo de Volatilidade Estocástica . . . . . . . . . . . . . 18

3 Modelos de Memória Longa $\quad 19$

3.1 Modelos de Memória Longa . . . . . . . . . . . . . . . . . . 19

3.1.1 Definição de Memória Longa . . . . . . . . . . . . . . . . . . 20 
3.1 .2 Medidas de Memória Longa . . . . . . . . . . . . . . . . 20

3.1 .3 Ruído Branco Fracionário . . . . . . . . . . . . . . . . 22

3.1.4 Modelo ARFIMA $(\mathrm{p}, \mathrm{d}, \mathrm{q}) \ldots \ldots \ldots \ldots . \ldots \ldots$

3.1.5 Estimador de Geweke e Porter-Hudak . . . . . . . . . . . . 26

3.1.6 Estimador usando o Periodograma Suavizado . . . . . . . . . . . . 29

3.1.7 Estimador Truncado de Whittle . . . . . . . . . . . . 32

3.1.8 Modelo de Volatilidade Estocástica de Memória Longa . . . . . . . 33

4 Memória Longa Compartilhada 35

4.1 Processos com Memória Longa Compartilhada . . . . . . . . . . . . . . . 35

4.2 Teste de Correlação Canônica para MLC . . . . . . . . . . . . . . . . 37

5 Simulações $\quad 42$

5.1 Avaliação de Estimadores . . . . . . . . . . . . . . . . . . . . 42

5.1 .1 Conjunto 1: Processos ARMA . . . . . . . . . . . . . 43

5.1.2 Conjunto 2: Razão de Desvios e Memória Longa . . . . . . . . . . . 44

5.1.3 Conjunto 3: Variações no Termo de Memória Longa . . . . . . . . . 47

5.1.4 Conjunto 4: Variações no Termo de Memória Curta . . . . . . . . . 52

5.2 Avaliação do Teste de Memória Longa Compartilhada . . . . . . . . . . . . 57

5.2 .1 Conjunto 5: Teste MLC . . . . . . . . . . . . . 57

5.2.2 Conjunto 6: Teste MLC - Variações no Termo de Memória Longa • 58

5.2.3 Conjunto 7: Teste MLC - Variações no Termo de Memória Curta 59

5.3 Síntese de Conclusões . . . . . . . . . . . . . . . . . . . . . . 60

5.3.1 Desempenho dos Estimadores de $d \ldots \ldots$. . . . . . . 60

5.3 .2 Desempenho do Teste MLC . . . . . . . . . . . . . 61

6 Aplicações $\quad 63$ 
6.1 Velocidade do Vento . . . . . . . . . . . . . . . . . . 63

6.2 Bolsas de Hong Kong, Nova Zelândia e Singapura . . . . . . . . . . . . . 68

6.3 Bolsas de Valores do Brasil e Reino Unido . . . . . . . . . . . . . . . 71

$\begin{array}{lll}7 & \text { Conclusões e Resultados } & 77\end{array}$

$\begin{array}{ll}\text { A Tabelas das Simulações dos Estimadores de d } & \mathbf{7 9}\end{array}$

B Tabelas das Simulações do Teste MLC $\quad 92$

$\begin{array}{lr}\text { Referências } & 100\end{array}$ 


\section{Introdução}

Nos estudos estatísticos de determinadas séries observadas no tempo, verifica-se que existe uma dependência entre as observações, e esta decai hiperbólicamente com o tempo. Em outras palavras, o valor atual da série depende de muitos valores passados ou defasados da mesma, e a correlação entre as observações possui um decaimento hiperbólico conforme aumenta-se a defasagem. Séries que apresentam essa característica são denominadas séries com persistência ou memória longa.

Os primeiros estudos com o objetivo de compreender as propriedades de séries com memória longa foram realizados por Hurst(1951, 1956, 1957) e Lawrance e Kottegoda (1977), principalmente na área de climatologia e hidrologia. A partir de 1980 os modelos de memória longa também começaram a ser utilizados na área econométrica para a modelagem de taxas de juros e inflação, e atualmente vem sendo amplamente aplicados na modelagem da volatilidade de ativos financeiros ou transformações de potências da série de retornos (Ding, Granger e Engle, 1993). O tempo de "memória" ou dependência das observações é quantificado através do parâmetro de memória longa, geralmente denotado por $d$. Para valores de $d=0$, dizemos que a série não possui memória longa, para $d>0$ a série é persistente, e para $d<0$ a série é antipersistente (Diebolt e Guiraud, 2000).

Atualmente, a identificação e modelagem de fatores comuns vem ganhando importância na área financeira. Deseja-se avaliar, por exemplo, se o valor da taxa de câmbio e o preço de determinadas ações são regidos por componentes comuns (Baillie, 1996). Dessa forma, refina-se a avaliação da influência de alterações macroeconômicas em ativos financeiros, além de facilitar a realização de previsões.

Um dos objetivos deste trabalho é a avaliação de estimadores do parâmetro de memória 
longa no contexto de processos de memória curta adicionados à processos persistentes. Os estimadores a serem avaliados são o estimador de Geweke e Porter-Hudak $\left(\hat{d}_{G P H}\right)$, o estimador utilizando o periodograma suavizado $\left(\hat{d}_{S}\right)$ e o estimador truncado de Whittle $\left(\hat{d}_{T W}\right)$. Além disso, também há interesse na avaliação da performance do teste de memória longa compartilhada através da análise de correlação canônica (Ray e Tsay, 1997), que tem como objetivo testar se duas séries temporais são regidos pelos mesmos componentes de memória longa. Nos capítulos seguintes, veremos que a estimação de $d$ e os teste de memória longa compartilhada estão fortemente relacionados.

O capítulo 1 apresenta algumas definições e conceitos básicos na modelagem de séries temporais. Estes conceitos serão amplamente utilizados nos capítulos subseqüentes. Uma síntese dos modelos clássicos de séries temporais pode ser encontrada no capítulo 2 , onde são descritos o conceito de filtros, modelos para séries estacionárias e séries nãoestacionárias, modelos heterocedásticos condicionais e uma introdução à modelagem de séries financeiras.

No capítulo 3 são descritos diversos estudos relacionados a processos de memória longa, tais como definições, métodos de identificação, estimação, propriedades e características importantes desses processos. A metodologia dos três estimadores do parâmetro de persistência a serem avaliados encontram-se neste capítulo. No último tópico, definimos o modelo de volatilidade estocástica de memória longa (Breidt, Crato e de Lima, 1998), que será utilizado em aplicações práticas.

O teste de memória longa compartilhada (MLC) através da análise de correlação canônica é apresentado no capítulo 4. Este teste, basicamente, utiliza a análise de correlação canônica para verificar se a matriz de covariância cruzada de séries temporais multivariadas possui posto completo para lags grandes. Se a matriz não possuir posto completo, a metodologia é utilizada para encontrar vetores de combinações lineares das séries, de forma que essas combinações não possuam memória longa. O posto da matriz é testado utilizando as estatísticas $T$ e $T^{*}$, cujos comportamentos serão avaliados neste trabalho.

A avaliação dos estimadores do parâmetro de persistência e do teste MLC é feita através de simulações, cujos resultados são apresentados no capítulo 5. As tabelas correspondentes às simulações podem ser encontradas nos apêndices A e B.

Aplicações sobre dados reais das metodologias apresentadas podem ser encontradas no capítulo 6. Apresentamos uma aplicação na área de climatologia para velocidade do vento 
em São Paulo e Piracicaba, e duas aplicações financeiras para a volatilidade dos índices das bolsas de valores de Hong Kong, Nova Zelândia, Singapura, Brasil e Reino Unido.

Por fim, serão apresentadas as conclusões e resultados deste trabalho, bem como os apêndices. 


\section{CAPÍtulo 1}

\section{Conceitos e Definições}

Neste capítulo serão introduzidas algumas definições e conceitos básicos para o estudo estatístico de séries temporais. Mais detalhes podem ser encontrados em Shumway e Stoffer(2000) e Morettin e Toloi(1987).

Uma série temporal é o conjunto de qualquer tipo de observações indexadas no tempo, tais como a temperatura em determinada hora e local, preços de ativos financeiros, índice de inflação(IPCA) etc. Em geral expressa-se uma série temporal por $X_{t}$, onde t indica o instante de tempo da observação. Os modelos utilizados em séries temporais são processos estocásticos, ou seja, seguem leis probabilísticas.

Os processos de séries temporais mais simples são processos denominados estacionários. Em termos práticos, verifica-se que os processos estacionários possuem média e variância constante ao longo do tempo, ou seja, as observações parecem flutuar em torno de um mesmo nível e com varibilidade constante. Existem duas formas de estacionariedade: a estrita e a fraca.

Definição 1.0.1. Um processo é denominado estritamente estacionário se todas as distribuições finito-dimensionais das variáveis aleatórias são idênticas para qualquer instante de tempo.

Definição 1.0.2. Um processo é denominado fracamente estacionário (ou estacionário de segunda ordem) se a esperança e a variância das variáveis aleatórias são constantes ao longo do tempo, se as variáveis possuem segundo momento finito e a função de autocorrelação só depende da defasagem e não do tempo.

Se um processo for gaussiano ele será determinado apenas pelo primeiro e segundo 
momento, dessa forma, se o processo for fracamente estacionário (ou de segunda ordem), ele será estritamente estacionário. Por simplicidade, consideraremos como processos estacionários, somente os processos fracamente estacionários.

Muitas vezes, deseja-se verificar se existe alguma dependência entre os valores atuais de uma série com seus valores passados, de forma a facilitar previsões. Uma ferramenta muito útil para quantificar essa dependência são as funções de autocovariância e autocorrelação da série.

Definição 1.0.3. A função de autocovariância $\gamma(h)$ de um processo estacionário é definida por

$$
\gamma(h)=\operatorname{Cov}\left(X_{t}, X_{t-h}\right)=E\left[X_{t} X_{t-h}\right]-E\left[X_{t}\right] E\left[X_{t-h}\right]
$$

e satisfaz as seguintes propriedades:

1. $\gamma(0)=\operatorname{var}\left(X_{t}\right)>0$;

2. $\gamma(0) \geq \gamma(h)$;

3. é simétrica, ou seja, $\gamma(-h)=\gamma(h)$;

4. é sempre positiva definida.

Definição 1.0.4. A função de autocorrelação $(\rho(h))$ de uma série temporal é definida por

$$
\rho(h)=\frac{\gamma(h)}{\operatorname{var}\left(X_{t}\right)}=\frac{\operatorname{Cov}\left(X_{t}, X_{t-h}\right)}{\operatorname{var}\left(X_{t}\right)},
$$

Para séries observadas, em geral não se conhecem os valores verdadeiros dos termos acima, dessa forma, na prática utiliza-se um estimador para $\rho(h)$, dado por

$$
\begin{aligned}
\hat{\rho}(h) & =\sum_{t=1}^{N} \frac{\left(x_{t}-\bar{x}\right)\left(x_{t-h}-\bar{x}\right)}{N \hat{\sigma}^{2}}, \\
\hat{\sigma}^{2} & =\sum_{t=1}^{N} \frac{\left(x_{t}-\bar{x}\right)^{2}}{N} \\
\bar{x} & =\sum_{t=1}^{N} \frac{x_{t}}{N} .
\end{aligned}
$$

Suponha agora que temos duas séries temporais estacionárias $X_{t}$ e $Y_{t}$. Seria interessante se pudessemos encontrar uma forma de mensurar não só a dependência dos valores 
atuais das duas séries, mas também entre seus valores defasados. Com esse objetivo, foram definidas as funções de covariância e correlação cruzadas.

Definição 1.0.5. A função de covariância cruzada $\left(\gamma_{X Y}(h)\right)$ é dada por

$$
\gamma_{X Y}(h)=\operatorname{Cov}\left(X_{t}, Y_{t-h}\right)=E\left[X_{t} Y_{t-h}\right]-E\left[X_{t}\right] E\left[Y_{t-h}\right] .
$$

Definição 1.0.6. A função de correlação cruzada $\left(\rho_{X Y}(h)\right)$ é dada por

$$
\rho_{X Y}(h)=\frac{\gamma_{X Y}}{\sqrt{\gamma_{X}(0) \gamma_{Y}(0)}} .
$$

Na prática, como não se conhece os verdadeiros valores dos termos acima, utiliza-se um estimador para $\hat{\rho}_{X Y}(h)$, dado por

$$
\rho_{\hat{X} Y}(h)=\sum_{t=1}^{N} \frac{\left(x_{t}-\bar{x}\right)\left(y_{t-h}-\bar{y}\right)}{N \hat{\sigma}_{X} \hat{\sigma}_{Y}} .
$$

Um outro instrumento para avaliar a dependência de uma série com seus valores passados foi proposto por Box, Jenkins e Reisel (1994), e é denominada a função de autocorrelação parcial (f.a.c.p.). Seja $\phi_{k j}$ o $j$-ésimo coeficiente de um modelo $\operatorname{AR}(k)$, de tal modo que $\phi_{k k}$ é o coeficiente correspondente ao termo da $k$-ésima defasagem da série. A quantidade $\phi_{k k}$ é considerada uma função de $k$ e é denominada função de autocorrelação parcial de uma série.

Um artifício freqüentemente utilizado é aplicar a transformação de Fourier aos dados de forma a descrever a série não mais no domínio de tempo mas da freqüência. A vantagem na utilização dessa transformação é que eliminam-se os problemas inerentes à correlação serial, pois os componentes (funções senos e cossenos) são ortogonais.

Definição 1.0.7. O espectro ou função densidade espectral $(f(\lambda))$ de uma série $X_{t}$ é definido como sendo a transformada de Fourier da função de autocovariância do processo, ou seja

$$
f(\lambda)=\frac{1}{2 \pi} \sum_{h=-\infty}^{\infty} \gamma(h) \exp (-i \lambda h)
$$

se $\sum_{h=-\infty}^{\infty}|\gamma(h)|<\infty$. Assim, a transformação inversa é

$$
\gamma(h)=\int_{w=-\pi}^{\pi} f(w) \exp (i w h) d w .
$$


Teorema 1.0.1. Seja $X_{t}$ um processo da forma:

$$
X_{t}=\sum_{s=1}^{\infty} c_{s} a_{t-s}
$$

onde $c_{s}$ é uma série de constantes com $\sum_{s=1}^{\infty} c_{s}<\infty$ e $a_{t}$ uma série temporal. Em outras palavras, temos que a série $X_{t}$ é uma série filtrada de $a_{t}$. Seja a função de transferência $A(\lambda)$, tal que

$$
A(\lambda)=\sum_{s=-\infty}^{\infty} c_{s} \exp (-i s \lambda) .
$$

Se $f_{a}(\lambda)$ é o espectro do processo $a_{t}$, então o espectro $\left(f_{X}(\lambda)\right)$ do processo $X_{t}$ é dado por

$$
f_{X}(\lambda)=|A(\lambda)|^{2} f_{a}(\lambda)
$$

Como em aplicações práticas a função de autocovariância do processo não é conhecida, em geral utiliza-se uma estimativa para função densidade espectral. Um estimador freqüentemente utilizado é denominado periodograma e foi introduzido por Schuster (1906). Antes de definirmos o periodograma, considere as transformações senos e cossenos de uma série $X_{t}$ na freqüência $(\lambda)$.

Definição 1.0.8. A transformação cosseno $\left(X_{c}(\lambda)\right)$ é definida pela expressão

$$
X_{c}(\lambda)=n^{-\frac{1}{2}} \sum_{t=1}^{n} X_{t} \cos (2 \pi t \lambda .) .
$$

Definição 1.0.9. A transformação seno $\left(X_{s}(\lambda)\right)$ é definida pela expressão

$$
X_{s}(\lambda)=n^{-\frac{1}{2}} \sum_{t=1}^{n} X_{t} \operatorname{sen}(2 \pi t \lambda) .
$$

Definição 1.0.10. Considere as transformações senos e cossenos de uma série $X_{t}$. $O$ periodograma é um estimador do espectro e é definido por

$$
I(\lambda)=X_{c}^{2}(\lambda)+X_{s}^{2}(\lambda)
$$


Definição 1.0.11. A transformada discreta de Fourier de uma série $X_{t}$ de tamanho $n$ é dada por

$$
\xi(\lambda)=n^{-\frac{1}{2}} \sum_{t=1}^{n} X_{t} \exp (-2 \pi i \lambda t)
$$

onde $\lambda=k / n$, para $k=0,1, \ldots, n-1$.

Pode se verificar que o periodograma também pode ser representado por

$$
I(\lambda)=|\xi(\lambda)|^{2}
$$

Verifica-se que o periodograma é assintóticamente não viesado, porém possui variância $f^{2}(\lambda)$ (Brillinger, 1981). Portanto, o periodograma é um estimador não consistente para a função densidade espectral. Resultados assintóticos mostram que as transformações senos e cossenos tendem conjuntamente para variáveis com distribuição normal com média zero e variância $f(\lambda) / 2$. Dessa forma, verifica-se uma propriedade importante do periodograma, dada por

$$
\frac{2(I(\lambda))}{f(\lambda)} \sim \chi_{2}^{2}
$$

Utilizando a propriedade acima, é possível a construção de intervalos de confiança, testes de hipóteses, e diversas outras ferramentas no domínio da freqüência. 
CAPÍtulo 2

\section{Modelos Clássicos de Séries}

\section{Temporais}

Neste capítulo, serão apresentados alguns modelos tradicionais utilizados em séries temporais. Primeiramente, será introduzido o conceito de Processo Linear. Em seguida, serão apresentados modelos da família ARIMA introduzidos por Box e Jenkins (1994), modelos heterocedásticos da família GARCH propostos por Engle(1982) e volatilidade estocástica desenvolvido por Taylor $(1980,1986)$ e outros. Tais modelos são freqüentemente utilizados na modelagem de séries temporais financeiras, pois são aplicáveis à previsão de retornos e volatilidades.

\subsection{Processo Linear}

A classe de processos lineares em séries temporais possui uma grande abrangência de modelos, permitindo a modelagem de grande parte das séries estacionárias.

Um processo linear pode ser definido como a saída de um filtro linear, cuja entrada é um processo ruído branco, ou seja,

$$
\begin{aligned}
X_{t} & =\mu+\psi_{0} w_{t}+\psi_{1} w_{t-1}+\psi_{2} w_{t-2}+\ldots \\
& =\mu+\sum_{j=0}^{\infty} \psi_{j} w_{t-j}
\end{aligned}
$$

onde $w_{t}$ 's são ruídos branco com média zero e variância $\sigma^{2}$.

Sob determinadas condições, o processo pode ser representado por uma regressão de 
observações passadas da série , ou seja,

$$
\begin{aligned}
\tilde{X}_{t} & =\phi_{1} \tilde{X}_{t-1}+\phi_{2} \tilde{X}_{t-2}+\ldots+\phi_{p} \tilde{X}_{t-p}+\ldots+w_{t} \\
& =\sum_{j=1}^{\infty} \phi_{j} \tilde{X}_{t-j}+w_{t}
\end{aligned}
$$

onde $\tilde{X}=X_{t}-\mu$.

A função de autocovariância do processo é dada por

$$
\gamma_{X}(h)=\sigma^{2} \sum_{j=0}^{\infty} \psi_{j+h} \psi_{j} .
$$

A função densidade espectral do processo é dada por

$$
f_{x}(\lambda)=2 \sigma^{2} \Psi\left(e^{-i \lambda}\right) .
$$

onde $\Psi(B)=1+\psi_{1} B+\psi_{2} B^{2}+\ldots$

Proposição 2.1.1. Um processo linear será estacionário se

$$
\sum_{j=0}^{\infty} \psi_{j}^{2}<\infty
$$

\section{Propriedades:}

a) Se $X_{t}$ é um processo linear estacionário com função de autocorrelação $\rho_{X}(h)$, $E\left(w_{t}^{4}\right)<\infty$, e $w_{t}$ é um ruído branco, para cada $h=1,2, \ldots$

$$
\hat{\rho}_{X}(h) \stackrel{D}{\longrightarrow} N\left(\rho_{X}(h), \tau^{2}\right),
$$

onde $\tau^{2}$ é uma função de $\left.\rho_{X}(h)\right)$ e $\hat{\rho}_{X}(h)$ é a função de autocorrelação amostral.

b) Se $X_{t}$ é um processo ruído branco, então $\rho(h)=0$ para $h \neq 0, \sigma_{\hat{\rho}(h)}=1 / \sqrt{n}$ e

$$
\hat{\rho}_{X}(h) \stackrel{D}{\longrightarrow} N(0,1 / \sqrt{n}),
$$

c) Se $X_{t}$ e $Y_{t}$ forem conjuntamente não-correlacionados e pelo menos um dos processos for ruído branco, então $\hat{\rho}_{X Y}(h) \stackrel{D}{\longrightarrow} N(0,1 / \sqrt{n})$

\subsection{Modelos da família $\operatorname{ARIMA}(\mathrm{p}, \mathrm{d}, \mathrm{q})$}




\subsubsection{Modelo Autoregressivo}

Observando a função de autocorrelação de algumas séries temporais, verifica-se que existe dependência entre o valor atual da série e seus valores passados. Além disso, nota-se que esta dependência é cada vez menor para pontos mais distantes no tempo. Portanto, é natural tentar prever o valor de $X_{t}$ através de uma regressão linear sobre $p$ valores passados da série.

Um modelo autoregressivo de ordem $p$, é um caso particular da representação (2.2), pois a partir do índice $p+1$ os coeficientes são iguais zero. Denota-se o processo por $A R(p)$ e é definido por

$$
\tilde{X}_{t}=\phi_{1} \tilde{X}_{t-1}+\phi_{2} \tilde{X}_{t-2}+\ldots+\phi_{p} \tilde{X}_{t-p}+a_{t}
$$

onde $a_{t}$ é ruído branco com média nula e variância $\sigma^{2}$. O processo também pode ser representado, utilizando-se o operador $B$ (backward)

$$
\left(1-\phi_{1} B-\phi_{2} B^{2}-\ldots-\phi_{p} B^{p}\right) \tilde{X}_{t}=a_{t},
$$

$\mathrm{Ou}$

$$
\phi(B) \tilde{X}_{t}=a_{t},
$$

onde $B \tilde{X}_{t}=\tilde{X}_{t-1}$ e $\phi(B)$ é denominado polinômio autoregressivo.

Proposição 2.2.1. Um processo autoregressivo será estacionário se as raízes do polinômio $\phi(B)$ estiverem fora do círculo unitário. Este processo sempre será invertível.

A função de autocovariância de ordem $k$ de um processo $A R(p)$ é dada por

$$
\gamma_{k}=\phi_{1} \gamma_{k-1}+\phi_{2} \gamma_{k-2}+\ldots+\phi_{p} \gamma_{k-p}
$$

e $\gamma_{0}=\operatorname{Var}\left(X_{t}\right), \operatorname{logo}$, a função de autocorrelação de ordem $k$ do processo é

$$
\rho_{k}=\phi_{1} \rho_{k-1}+\phi_{2} \rho_{k-2}+\ldots+\phi_{p} \rho_{k-p} .
$$

Observação 2.2.1. A função de autocorrelação de um $A R(p)$ decai exponencialmente para zero ou através de senóides amortecidas. Além disso, a função de autocorrelação parcial de um $A R(p)$ é nula para lags maiores que $p$. Portanto uma forma de identificar estes processos é a análise das funções de autocorrelação e autocorrelação parcial amostrais. 


\subsubsection{Modelo de Médias Móveis}

A caracterização de um processo de médias móveis é menos intuitiva que a de um processo $A R$, pois consiste em uma regressão onde as variáveis explicativas são os valores passados dos erros aleatórios. Em outras palavras, um modelo de médias móveis de ordem $q$ é um caso particular da representação (2.1), onde apenas os coeficientes a partir do índice $q+1$ são iguais à zero. Denota-se este processo por $M A(q)$, e é definido por

$$
\tilde{X}_{t}=a_{t}-\theta_{1} a_{t-1}-\theta_{2} a_{t-2}-\ldots-\theta_{q} a_{t-q},
$$

onde $a_{t}$ é ruído branco com média nula e variância $\sigma^{2}$. O processo também pode ser descrito, utilizando-se o polinômio B(backward)

$$
\tilde{X}_{t}=\left(1-\theta_{1} B-\theta_{2} B^{2}-\ldots-\theta_{q} B^{q}\right) a_{t}
$$

$\mathrm{Ou}$

$$
\tilde{X}_{t}=\theta(B) a_{t}
$$

onde $\theta(B)$ é denominado operador de médias móveis.

Proposição 2.2.2. Um processo de médias móveis é sempre estacionário. Para que o processo seja invertível, é necessário que as raízes do polinômio $\theta(B)$ estejam fora do círculo unitário.

A função de autocovariância de um processo $M A(q)$ é dada por

$$
\gamma_{k}=\left(-\theta_{k}+\theta_{1} \theta_{k+1}+\theta_{2} \theta_{k+2}+\ldots \theta_{q-k} \theta_{q}\right) \sigma_{a}^{2},
$$

para $k=1, . ., q$, caso contrário $\gamma_{k}=0$.

A função de autocorrelação do processo é

$$
\rho_{k}=\frac{\left(-\theta_{k}+\theta_{1} \theta_{k+1}+\theta_{2} \theta_{k+2}+\ldots \theta_{q-k} \theta_{q}\right)}{\left(1+\theta_{1}^{2}+\ldots+\theta_{q}^{2}\right)} .
$$

Observação 2.2.2. A função de autocorrelação de um $M A(q)$ é nula para lags maiores que $q$. A função de autocorrelação parcial decai para zero exponencialmente. Portanto, este processo pode ser identificado analisando as funções de autocorrelação e autocorrelação parcial amostrais. Note que a f.a.c. e f.a.c.p. deste processo possuem comportamento inverso ao processo autoregressivo. 


\subsubsection{Modelo Autoregressivo e de Médias Móveis}

Um modelo autoregressivo e de médias móveis é uma união de processos autoregressivos de ordem $p$ e processos de médias móveis de ordem $q$. É freqüentemente utilizado por ser mais parcimonioso que os dois modelos anteriores. O processo é denotado por $\operatorname{ARMA}(p, q)$ e é definido da seguinte forma:

$$
\tilde{X}_{t}-\phi_{1} \tilde{X}_{t-1}-\ldots-\phi_{p} \tilde{X}_{t-p}=a_{t}-\theta_{1} a_{t-1}-\ldots-\theta_{q} a_{t-q},
$$

onde $a_{t}$ é ruído branco com média nula e variância $\sigma^{2}$. O processo também pode ser representado, utilizando-se o operador $B$ (backward)

$$
\phi(B) \tilde{X}_{t}=\theta(B) a_{t},
$$

Proposição 2.2.3. Um processo de médias móveis será estacionário se as raízes do polinômio $\phi(B)$ estiverem fora do círculo unitário. Para que o processo seja invertível é necessário que as raízes do polinômio $\theta(B)$ estejam fora do círculo unitário.

A função de autocovariância de um processo $A R M A(p, q)$ é dada por

$$
\gamma_{k}=\phi_{1} \gamma_{k-1}+\ldots+\phi_{p} \gamma_{k-p}+\gamma_{\tilde{X} a}(k)-\theta_{1} \gamma_{\tilde{X} a}(k-1)-\theta_{q} \gamma_{\tilde{X} a}(k-q),
$$

onde $\gamma_{\tilde{X} a}(k)$ é a função de covariância cruzada entre $\tilde{X}_{t}$ e $a_{t}$. Como $\tilde{X}_{t-k}$ só depende de choque $a_{t}$ ocorridos até o instante $t-k$, obtemos:

$$
\gamma_{X a}(k)=\theta_{-k} \sigma_{a}^{2}
$$

se $k \leq 0$, caso contrário $\gamma_{X a}(k)=0$.

A função de autocorrelação do processo, para $k>q$ é

$$
\rho_{k}=\phi_{1} \rho_{k-1}+\phi_{2} \rho_{k-2}+\ldots+\phi_{p} \rho_{k-p} \text {. }
$$

Observação 2.2.3. A função de autocorrelação de um $A R M A(p, q)$ possui o mesmo comportamento de um $A R(p)$ para lags maiores que $q$, ou seja, decai exponencialmente. A função de autocorrelação parcial decai para zero exponencialmente a partir do lag $p$.

O espectro do processo $A R M A(p, q)$ é dada por

$$
f_{X}(\lambda)=\frac{\sigma_{a}^{2}\left|\theta\left(e^{-i \lambda}\right)\right|^{2}}{2 \pi\left|\phi\left(e^{-i \lambda}\right)\right|^{2}}
$$




\subsubsection{Modelo Autoregressivo e de Médias Móveis Integrado}

Na prática, muitas vezes observa-se séries não-estacionárias e que não possuem tendência determinística (como na forma polinomial, por exemplo), porém observa-se a estacionariedade na primeira diferença da série. Temos que um processo ARMA é estacionário se as raízes da equação característica $\phi(B)$ estiverem fora do círculo unitário. O modelo ARIMA foi proposto para a modelagem de processos na qual pelo menos uma das raízes se encontra sobre o círculo unitário. Processos dessa forma são denominados processos não-estacionários homogêneos.

Considere o seguinte modelo:

$$
\varphi(B) \tilde{X}=\theta(B) a_{t}
$$

onde $\varphi(B)$ é um operador não-estacionário, ou seja, pelo menos uma das raízes da equação características encontra-se sobre o círculo unitário. Pode-se também representar o modelo utilizando o operador diferença $\Delta$, da seguinte forma

$$
\phi(B) \Delta^{d} \tilde{X}_{t}=\theta(B) a_{t}
$$

onde $\Delta^{d}=(1-B)^{d}$.

Este processo é denominado autoregressivo e de médias móveis integrados denotado por $A R I M A(p, d, q)$. Verifica-se que este modelo assume que a d-ésima diferença da série pode ser representada por um processo $A R M A(p, q)$ estacionário e invertível. Basicamente, existem três formas explícitas de se representar um modelo $A R I M A(p, d, q)$, que serão apresentados a seguir.

A primeira representação é através de choques aleatórios. Este modelo representa o processo $X_{t}$ como saída de um filtro linear cuja entrada são ruídos branco. O modelo é dado por:

$$
\tilde{X}_{t}=\psi(B) a_{t} .
$$

Multiplicando-se ambos os lados da equação por $\varphi(B)$, obtemos

$$
\varphi(B) \tilde{X}_{t}=\varphi(B) \psi(B) a_{t},
$$

mas temos também que

$$
\varphi(B) \tilde{X}_{t}=\theta(B) a_{t},
$$


$\log 0$

$$
\varphi(B) \psi(B)=\theta(B)
$$

Portanto, para obtermos os coeficientes $\psi$ do filtro linear, basta expandir os polinômios

$$
\left(1-\varphi_{1} B-\ldots-\varphi_{p+d} B^{p+d}\right)\left(1+\psi_{1} B+\psi_{2} B^{2}+\ldots\right)=\left(1-\theta_{1} B-\ldots-\theta_{q}^{q}\right) .
$$

Uma segunda representação é dada utilizando-se equações de diferenças, onde expressase o valor atual de $X_{t}$ como função dos valores passados de $X_{t}$ e valores passados dos $a_{t}$. O modelo é representado por

$$
\tilde{X}=\varphi_{1} \tilde{X}_{t-1}+\ldots+\varphi_{p+d} \tilde{X}_{t-p-d}-\theta_{t-1} a_{t-1}-\ldots-\theta_{t-q} a_{t-q}+a_{t} .
$$

O modelo também pode ser representado na forma invertida, onde o processo $X_{t}$ é representado como função dos valores passados de $X_{t}$ e do valor atual de $a_{t}$. O modelo é representado por

$$
\tilde{X}=\sum_{j=1}^{\infty} \pi_{j} \tilde{X}_{t-j}+a_{t}
$$

Os coeficientes $\pi$ são obtidos equacionando-se os coeficientes de B em

$$
\begin{aligned}
\varphi(B) & =\theta(B) \pi(B) \\
\left(1-\varphi_{1} B-\ldots-\varphi_{p+d} B^{p+d}\right) & =\left(1-\theta_{1} B-\ldots-\theta_{q} B^{q}\right)\left(1-\pi_{1} B-\pi_{2} B^{2}-\ldots\right) .
\end{aligned}
$$

\subsection{Modelos Heterocedásticos Condicionais}

Nos mercados financeiros existem diversos ativos no qual o capital pode ser investido. Exemplificando, suponha que ações de uma empresa $A$ sejam comercializadas na bolsa de valores. Cada ação (ativo) representa uma pequena quantidade do patrimônio da empresa e portanto possui um preço $P_{t}$ variável ao longo do tempo, pois está sujeito às flutuações do mercado.

O retorno simples de $k$ períodos de um ativo financeiro é definido por

$$
R_{t}[k]=\frac{P_{t}}{P_{t-k}}-1
$$


Note que o retorno simples é simplesmente a variação percentual do preço do ativo em um determinado período de tempo de amplitude $k$.

A volatilidade de um ativo financeiro é definida como o potencial de variabilidade do preço em um determinado período de tempo. Em outras palavras, se o preço das ações da empresa A apresenta uma variabilidade muito grande, dizemos que o ativo possui grande volatilidade. Nos mercados financeiros, a volatilidade de um ativo é de grande importância, pois mede o risco que um investidor assume ao comprá-lo. Isto vem do fato de que se o preço do ativo possui grande potencial para subir, também possui grande potencial para cair. A forma mais usual de se quantificar a volatilidade de um ativo é através do desvio-padrão da série de retornos, pois quanto maior a variabilidade dos retornos, maior será a variabilidade da série de preços.

Empiricamente, verifica-se que a série de retornos não possui distribuição de probabilidade normal, suposição em geral assumida para a estimação de modelos ARIMA (geralmente estimado pelo método da máxima verossimilhança). Porém, tal condição é satisfeita para a série de log-retornos. O log-retorno de $k$ períodos de um ativo financeiro é definido por:

$$
r_{t}[k]=\ln \left(\frac{P_{t}}{P_{t-k}}\right),
$$

ou seja, os retornos de grande parte dos ativos financeiros, possui distribuição de probabilidade lognormal. Outra vantagem na utilização de log-retornos é que o log-retorno de um ativo em k períodos, é simplesmente a soma dos log-retornos de cada período.

Além disso, temos que a variabilidade da série de log-retornos não é constante. Determinadas características da volatilidade podem ser observadas em séries de ativos financeiros, principalmente de ações de bolsas de valores, entre elas,

- existência de conglomerados de volatilidade;

- retornos altos são em geral seguidos por novos retornos altos;

- quando o nível de preço de determinado ativo está baixo aumenta-se a incerteza dos investidores sobre o rumo do preço do ativo, impactando em um aumento na volatilidade da série de retornos. 


\subsubsection{Modelos da Família GARCH}

Os modelos da família $G A R C H$ foram propostos por Engle(1982) para a modelagem da taxa inflacionária britânica, e são modelos não-lineares, no que se refere a variância. Verifica-se que grande parte das séries financeiras apresentam conglomerados de volatilidades, ou seja, a volatilidade apresenta um comportamento inercial, de forma que suas variações se dão lentamente.

\section{Modelo GARCH}

Em geral, utiliza-se os modelos da família $A R I M A$ para a modelagem da função média da série de log-retornos da série conjuntamente com modelos da família $G A R C H$ para a modelagem da variância condicional. Um modelo $A R I M A(p, q)$ para a função média do $\log$-retorno $r_{t}$ é dado por:

$$
r_{t}=\phi_{0} \sum_{i=1}^{p} \phi_{i} r_{t-i}+a_{t}-\sum_{j=1}^{q} \theta_{j} a_{t-j},
$$

onde $a_{t}$ é ruído branco. Um modelo $G A R C H(r, s)$ para a volatilidade da série é dado por:

$$
\begin{aligned}
a_{t} & =\sigma_{t} \epsilon_{t}, \\
\sigma_{t}^{2} & =\alpha_{0}+\sum_{i=1}^{r} \alpha_{i} a_{t-i}^{2}+\sum_{j=1}^{s} \beta_{j} \sigma_{t-j}^{2},
\end{aligned}
$$

onde $\sum_{i=1}^{r} \alpha_{i}+\sum_{j=1}^{s} \beta_{j}<1$ e $\alpha_{i} \geq 0, \beta_{j} \geq 0$ para todo $i, j$.

Note que no modelo $G A R C H$ a variância é a soma ponderada de valores passados da volatilidade $\sigma_{t}^{2}$ e dos quadrados dos erros aleatórios $a_{t}^{2}$. Temos também que embora a série $a_{t}$ seja não-correlacionada com seus valores passados, apresenta dependência descrita por uma função quadrática. Portanto, uma forma de identificação destes modelos é observar a função de autocorrelação e função de autocorrelação parcial do quadrado da série padronizada.

\section{Modelo EGARCH e TARCH}

Observa-se que embora o modelo $G A R C H$ incorpore o comportamento inercial da volatilidade, o impacto de retornos negativos e positivos é modelado da mesma forma. Porém, empiricamente, retornos negativos são seguidos de acréscimo na volatilidade. $\mathrm{O}$ modelo $E G A R C H$ (Nelson, 1991) para a volatilidade foi construído de forma a incorporar distintamente retornos positivos e negativos, e é dado por:

$$
\log \left(\sigma_{t}^{2}\right)=\alpha_{0}+\sum_{i=1}^{r} \alpha_{i}\left|\epsilon_{t-i}\right|+\sum_{i=1}^{r} \gamma_{i} \epsilon_{t-i}+\sum_{j=1}^{s} \beta_{j} \sigma_{t-j}^{2},
$$


onde $\epsilon_{t-i}$ é ruído branco com distribuição normal padrão. Se pelo menos um dos coeficientes $\gamma_{i}$ for estatísticamente significante, verifica-se a existência do efeito de assimetria dos retornos na volatilidade.

Uma forma alternativa para se incorporar o impacto assimétrico dos retornos é dada pelo modelo TARCH (Glosten, Jagannathan e Runkle, 1993), definido por:

$$
\sigma_{t}^{2}=\alpha_{0}+\sum_{i=1}^{r} \alpha_{i} r_{t-i}^{2}+\sum_{i=1}^{r} \gamma_{i} r_{t-i}^{2} d_{t-i}+\sum_{j=1}^{s} \beta_{j} \sigma_{t-j}^{2},
$$

com $d_{t}=1$ se $r_{t}<0$, e zero caso contrário.

\subsubsection{Modelo de Volatilidade Estocástica}

O modelo de volatilidade estocástica, proposto por Taylor $(1980,1986)$ e outros, é uma forma alternativa aos modelos da familia $G A R C H$ para a modelagem do comportamento inercial da volatilidade dos retornos. Note que no modelo $G A R C H$ a volatilidade depende apenas de seus valores passados e dos valores passados da série de log-retornos, portanto, dada a informação até o instante $t-1$ a volatilidade no tempo $t$ é determinística. $\mathrm{O}$ modelo de volatilidade estocástica inclui um componente aleatório para a volatilidade, e é definido por:

$$
\begin{aligned}
\log \left(r_{t}^{2}\right) & =K+h_{t}+u_{t}, \\
h_{t} & =\alpha h_{t-1}+\nu_{t}
\end{aligned}
$$

onde $K=\log \left(\sigma^{2}\right)+E\left[\log \left(\epsilon_{t}^{2}\right)\right], u_{t}=\log \left(\epsilon_{t}^{2}\right)-E\left[\log \left(\epsilon_{t}^{2}\right)\right], \operatorname{com} \epsilon_{t} \sim N(0,1)$ e $\nu_{t} \sim N\left(0, \sigma_{\nu}\right)$ ruídos brancos.

Verifica-se que o modelo de volatilidade estocástica é um caso particular de um modelo de espaço de estados. Logo pode-se utilizar esta formulação para realizar a estimação dos parâmetros e previsão, através do filtro de Kalman por exemplo. 
CAPÍtulo 3

\section{Modelos de Memória Longa}

Verifica-se que a função de autocorrelação dos processos descritos no capítulo anterior possuem decaimento rápido para zero e em taxa exponencial ou geométrica(memória curta). Neste capítulo serão apresentados alguns modelos que possuem decaimento mais lento e em taxa hiperbólica da função de autocorrelação, denominados modelos de memória longa ou de integração fracionária. Em seguida, serão apresentados alguns testes e medidas para a persistência.

\subsection{Modelos de Memória Longa}

Os modelos de memória longa foram primeiramente desenvolvidos por estatísticos envolvidos na área de climatologia e hidrologia. Hurst $(1951,1956,1957)$ analisou diversas séries temporais geofísicas buscando compreender a persistência existente nas séries de vazão de água, para um melhor planejamento de reservatórios. Modelos estatísticos para a análise de vazão de rios foram sugeridos também por Lawrance e Kottegoda (1977), enquanto que Hipel e Mcleod (1978a,b) consideraram algumas aplicações na área climatológica, onde verifica-se a presença de memória longa nas séries de velocidade do vento e temperatura.

Somente na década de 1980 os modelos de memória longa começaram a ser utilizados na área econométrica, quando verificou-se que eram adequados para a previsão de determinados tipos de taxas de juros, inflação, etc. (Baillie, 1996). Provavelmente, um dos maiores sucessos foi a modelagem da volatilidade de ativos financeiros e transformações 
de potências da série de retornos.

\subsubsection{Definição de Memória Longa}

Em termos gerais, dizemos que uma série $y_{t}$ possui persistência ou memória longa, se $y_{t}$ é correlacionado com $y_{t-j}$ para valores grandes de $j$, de forma que a correlação decai hiperbolicamente conforme $j$ cresce. Formalmente, considere que $y_{t}$ é uma série temporal com função de autocorrelação $\rho_{j}$ no lag $j$. A condição de estacionariedade de qualquer processo é satisfeita se

$$
\lim _{n \rightarrow \infty} \sum_{j=-n}^{n} \rho_{j}^{2}<\infty
$$

Segundo McLeod e Hipel (1978), o processo possui memória longa se a quantidade

$$
\lim _{n \rightarrow \infty} \sum_{j=-n}^{n}\left|\rho_{j}\right|
$$

não for finita. Dizemos que o processo $y_{t}$ é integrado de ordem $d$, ou $I(d)$ se

$$
(1-B)^{d} y_{t}=u_{t},
$$

onde $B$ é operador backward, $-0,5<d<0,5$ e $u_{t}$ é um processo estacionário.

Para $0<d<0,5$ o processo possui memória longa, e observa-se que a função de autocorrelacão é positiva e decai hiperbólicamente. Alguns autores (Diebolt e Guiraud, 2002) consideram que para $-0,5<d<0$, o processo não possui memória longa, pois há alternação do sinal da função de autocorrelação. Dessa forma, este tipo de processo é denominado antipersistente, mas não está no escopo deste trabalho.

\subsubsection{Medidas de Memória Longa}

A primeira estatística para quantificar o efeito de memória longa foi proposta por Hurst (1951) e é denominada estatística $(R / S)$ (rescaled range), definida por

$$
R=\left[\max _{1 \leq j \leq T}\left(\sum_{t=1}^{j}\left(y_{t}-t \bar{y}\right)\right)-\min _{1 \leq j \leq T}\left(\sum_{t=1}^{j}\left(y_{t}-t \bar{y}\right)\right)\right] \frac{1}{S},
$$


onde $R$ é a amplitude, $\bar{y}$ é a média amostral, e

$$
S=\left(\frac{\sum_{t=1}^{T}\left(y_{t}-\bar{y}\right)^{2}}{T}\right)^{1 / 2} .
$$

é o desvio padrão amostral. Hurst(1951) mostrou que

$$
T^{-H}[R / S] \stackrel{P}{\rightarrow} \text { constante }
$$

quando $T \rightarrow \infty$, e $H$ é o denominado coeficiente de Hurst. Uma propriedade interessante da estatística $\mathrm{R} / \mathrm{S}$ é que

$$
\log [E(R / S)] \approx \text { constante }+H[\log (T)]
$$

Assim, o coeficiente de Hurst $H$ pode ser estimado por $\log \frac{R / S}{\log (T)}$, ou através do coeficiente de inclinação estimado de uma regressão entre $\log \frac{R}{S}$ e $\log (T)$ para valores distintos de T. Como um processo de memória curta possui um valor de $\mathrm{H}$ igual à 0,5 , séries com estimativas de $\mathrm{H}$ que excedem relevantemente 0,5 podem ser intepretadas como séries de memória longa.

Contudo, alguns estudos indicam que a estatística R/S possui sérias deficiências na presença de dados gerados pela combinação de processos de memória longa e processos de memória curta. Uma estatística modificada foi proposta por Lo(1991), e é definida por

$$
Q=\frac{R}{\sigma(q)}
$$

onde

$$
\sigma^{2}(q)=c_{0}+2 \sum_{j=1}^{q} w_{j}(q) c_{j},
$$

$c_{j}$ é a função de autocovariância amostral no lag j da série e $w_{j}$ é a janela de Bartlett com pesos

$$
w_{j}(q)=1-\left[\frac{j}{q+1}\right]
$$

para valores de $q<T$. A escolha de $q$ deve ser baseada na ordem dos processos de memória curta envolvidos. 


\subsubsection{Ruído Branco Fracionário}

Um dos processos mais simples de memória longa é o ruído branco fracionário, proposto independentemente por Granger (1980), Granger e Joyeux (1980) e Hosking (1981), e é definido por

$$
(1-B)^{d}\left(y_{t}-\nu\right)=\epsilon_{t}
$$

onde $E\left[\epsilon_{t}\right]=0, E\left[\epsilon_{t}^{2}\right]=\sigma^{2}, E\left(y_{t}\right)=\nu$, e $E\left[\epsilon_{t} \epsilon_{s}\right]=0$ para $s \neq t$ onde o parâmetro fracionário $d$ é não-inteiro. Sem perda de generalidade, vamos considerar $E\left(y_{t}\right)=\nu=0$. Verifica-se que este processo será estacionário se $d<0,5$ e será invertível se $d>-0,5$.

O modelo pode ser escrito como um processo autoregressivo de ordem infinita

$$
y_{t}=\sum_{k=1}^{\infty} \pi_{k} y_{t-k}+\epsilon_{t},
$$

onde os coeficientes $\pi_{k}$ podem ser obtidos através da expansão binomial,

$$
(1-B)^{d}=1-d B+\frac{d(d-1) B^{2}}{2 !}-\frac{d(d-1)(d-2) B^{3}}{3 !}+\ldots,
$$

para qualquer $d>-1 \in \mathbf{R}$. Ou seja, o coeficiente autoregressivo no lag $k$ é dado por

$$
\begin{aligned}
\pi_{k} & =\frac{d(d-1)(d-2) \ldots(d-k+1)(-1)}{k !} \\
& =\frac{(-d)(1-d)(2-d) \ldots(k-1-d)}{k !} \\
& =\frac{\Gamma(k-d)}{\Gamma(-d) \Gamma(k+1)} .
\end{aligned}
$$

Um ruído branco com integração fracionária também pode ser escrito na forma de choques aleatórios (decomposição de Wold), dada por

$$
\begin{aligned}
y_{t} & =\sum_{k=0}^{\infty} \psi_{k} \epsilon_{t-k} \\
& =(1-B)^{-d} \epsilon_{t} \\
& =\left(1+d B+\frac{d(d+1) B^{2}}{2 !}+\frac{d(d+1)(d+2) B^{3}}{3 !}+\ldots\right) \epsilon_{t} .
\end{aligned}
$$

Portanto, temos que os pesos $\psi_{k}$ são dados por

$$
\psi_{k}=\frac{\Gamma(k+d)}{\Gamma(d) \Gamma(k+1)}
$$


A função de autocovariância do processo é

$$
\begin{aligned}
& \gamma_{0}=\sigma^{2} \frac{\Gamma(1-2 d)}{\Gamma^{2}(1-d)} \\
& \gamma_{k}=\sigma^{2} \frac{\Gamma(k+d) \Gamma(1-2 d)}{\Gamma(k+1-d) \Gamma(1-d) \Gamma(d)},
\end{aligned}
$$

e a função de autocorrelação é dada pela expressão

$$
\begin{aligned}
\rho_{1} & =\frac{d}{1-d}, \\
\rho_{2} & =\frac{d(1+d)}{(1-d)(2-d)}, \\
\rho_{k} & =\frac{\Gamma(k+d) \Gamma(1-d)}{\Gamma(k-d+1) \Gamma(d)} .
\end{aligned}
$$

A Figura 3.1 ilustra o decaimento da função de autocorrelação de um ruído branco fracionário $\operatorname{com} d=0.4$.

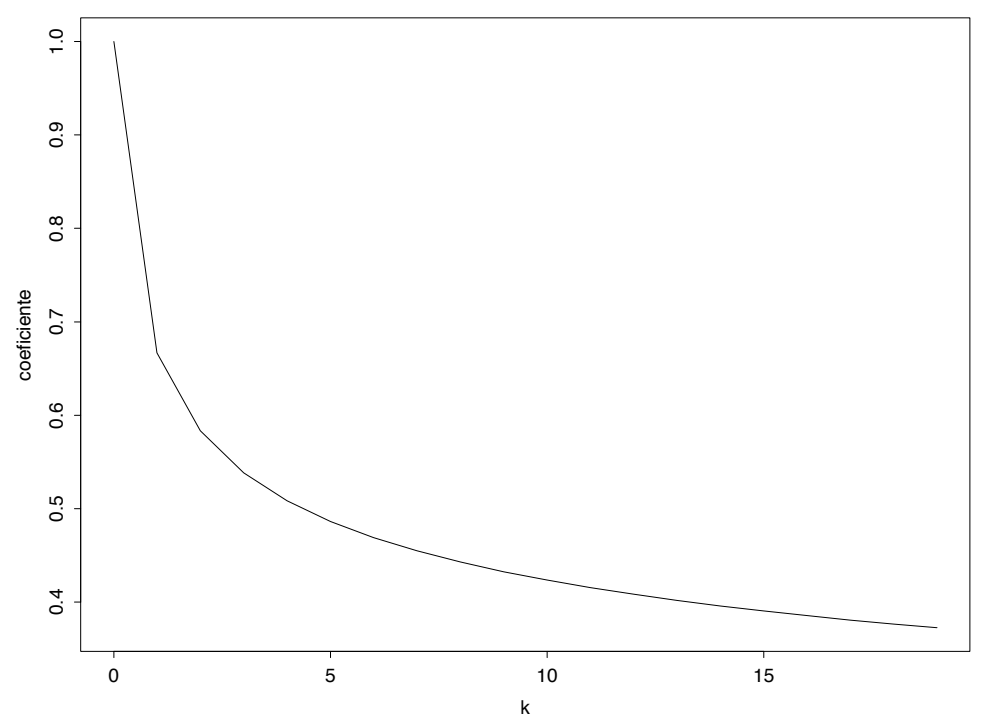

Figura 3.1: Função de Autocorrelação $\left(\rho_{k}\right)$ para $d=0.4$

O espectro de um processo ruído branco fracionário é dado por

$$
\begin{aligned}
f(\lambda) & =\frac{\sigma^{2}}{2 \pi}\left|1-e^{-i \lambda}\right|^{-2 d} \\
& =\frac{\sigma^{2}}{2 \pi}\left[2 \operatorname{sen}\left(\frac{\lambda}{2}\right)\right]^{-2 d} .
\end{aligned}
$$


Utilizando a aproximação de Stirling quando $k \rightarrow \infty$, temos que $\frac{\Gamma(k+a)}{\Gamma(k+b)} \approx k^{a-b}$. Assim podemos fazer as seguintes aproximações:

$$
\begin{aligned}
\psi_{k} & \approx \Gamma(d)^{-1} k^{d-1} \approx c_{1} k^{d-1} \\
\pi_{k} & \approx \Gamma(-d)^{-1} k^{-d-1} \approx c_{2} k^{-d-1} \\
\rho_{k} & \approx \frac{\Gamma(1-d)}{\Gamma(d)} k^{2 d-1} \approx c_{3} k^{2 d-1}
\end{aligned}
$$

Uma propriedade interessante dos processos ruído branco fracionários é que função densidade espectral nas baixas freqüências pode ser aproximada por

$$
f(\lambda) \approx \lambda^{-2 d}
$$

Note que existe um forte contraste entre a função densidade espectral deste processo e a de séries com $d=0$ que são próximas de zero nas baixas freqüências. Veremos mais tarde que alguns autores aproveitam-se deste fato para a determinação de métodos de detecção de memória longa.

\subsubsection{Modelo ARFIMA(p,d,q)}

O modelo autoregressivo e de médias móveis com integração fracionária foi introduzido por Granger e Joyeux (1980), Granger (1980) e Hosking (1981) e caracteriza-se pela sua grande flexibilidade. Um modelo $\operatorname{ARFIMA}(p, d, q)$ é definido por

$$
\phi(B)(1-B)^{d}\left(y_{t}-\mu\right)=\theta(B) \epsilon_{t},
$$

onde $d$ denota o parâmetro de diferença fracionária e $\epsilon_{t}$ é um ruído branco com variância $\sigma^{2}$. Verifica-se que a função de autocorrelação apresenta um lento decaimento para zero em taxa hiperbólica. Nas baixas freqüencias com $d>0$, uma aproximação para o espectro é $f(\lambda) \approx \lambda^{-2 d}$. Temos que o processo possui variância infinita se $d>0,5$.

Note que para o modelo ARMA tradicional é um caso particular do modelo ARFIMA, quando $d=0$. Verifica-se também que o efeito o parâmetro $d$ para observações mais distantes decai hiperbólicamente, mas os efeitos dos parâmetros $p$ e $q$ decai exponencialmente. Portanto, na modelagem de processos $\operatorname{ARFIMA}(p, d, q)$, o parâmetro $d$ deve se escolhido de forma a explicar a estrutura de correlação nos lags mais altos, e os parâmetros $p$ e $q$ 
nos lags baixos. Logo, a estrutura de autocorrelação de um processo $\operatorname{ARFIMA}(p, d, q)$ é muito próxima a de um ruído branco fracionário no lag $\mathrm{j}$, para valores de $\mathrm{j}$ suficientemente grandes.

Proposição 3.1.1. Se $\left\{X_{t}\right\}$ é um processo ARFIMA(p,d,q), então

1) O processo será estacionário se $d<0,5$ e as raízes o polinômio $\phi(B)$ estiverem fora do círculo unitário.

2) O processo será invertível se $d>-0,5$ e as raízes o polinômio $\theta(B)$ estiverem fora do círculo unitário.

A representação na forma de choques aleatórios do processo é dada por

$$
X_{t}=\psi(B) \epsilon_{t}=\sum_{j=0}^{\infty} \psi_{j} \epsilon_{t-j}
$$

onde $\psi_{0}=1$ e $\psi(B)=(1-B) \frac{\theta(B)}{\phi(B)}$. Portanto, temos

$$
f_{X}(\lambda)=|A(\lambda)|^{2} f_{\epsilon}(\lambda)
$$

onde

$$
\begin{aligned}
A(\lambda) & =\sum_{t=0}^{\infty} \Psi_{t} e^{-i \lambda t}=\psi\left(e^{-i \lambda}\right)=\left(1-e^{-i \lambda}\right)^{-d} \frac{\theta\left(e^{-i \lambda}\right)}{\phi\left(e^{-i \lambda}\right)} \\
|A(\lambda)|^{2} & =\left(2 \operatorname{sen}\left(\frac{\lambda}{2}\right)\right)^{-2 d} \frac{\left|\theta\left(e^{-i \lambda}\right)\right|^{2}}{\left|\phi\left(e^{-i \lambda}\right)\right|^{2}}
\end{aligned}
$$

Portanto, temos que o espectro de um processo ARFIMA pode ser representado por

$$
f_{X}(\lambda)=\left(2 \operatorname{sen}\left(\frac{\lambda}{2}\right)\right)^{-2 d} \frac{\left|\theta\left(e^{-i \lambda}\right)\right|^{2}}{\left|\phi\left(e^{-i \lambda}\right)\right|^{2}} \frac{\sigma_{\epsilon}^{2}}{2 \pi} .
$$

Sowell(1986, 1992a) mostrou que a função de autocovariância deste processo é da forma

$$
\gamma_{k}=\sigma^{2} \sum_{j=1}^{p} \xi_{j} \sum_{n=0}^{q} \sum_{m=0}^{q} \theta_{n} \theta_{m} C\left(d, d, p+n-m-k, \lambda_{j}\right),
$$

onde $\lambda_{j}$ é a j-ésima raiz do polinômio $\phi(B)$ e

$$
\begin{aligned}
\xi_{j} & =\left\{\lambda_{j} \Pi_{i=1, p}\left(1-\rho_{i} \rho_{j}\right) \Pi_{i=1, p ; k \neq i}\left(\rho_{i}-\rho_{k}\right)\right\}^{-1} \\
C(w, v, k, \rho) & =G(w, v, k)\left[\rho^{2 p} F(v+k, 1 ; 1-w+k ; ; \rho)+\right. \\
& +F(w-k, 1 ; 1-v-k ; \rho)-1] \\
G(w, v, k) & =\frac{\Gamma(1-w-v) \Gamma(v+k)}{\Gamma(1-w+k) \Gamma(1-v) \Gamma(v)} .
\end{aligned}
$$


e $F(a, b ; c ; x)$ é a função hipergeométrica

$$
F(a, b ; c ; x)=1+\frac{a b}{c} x+\frac{a(a+1)(b(b+1)}{c(c+1) 1} x^{2}+\ldots
$$

\subsubsection{Estimador de Geweke e Porter-Hudak}

O método da regressão espectral foi proposto por Geweke e Porter-Hudak (1983) e é freqüentemente utilizado para a estimação do parâmetro $d$ de um processo de memória longa $I(d)$. Este método baseia-se na relação entre a função densidade espectral de um processo ARFIMA(p,d,q) e suas respectivas coordenadas harmônicas.

Se $\left\{X_{t}\right\}$ é um processo $A R F I M A(p, d, q)$ com $E\left[X_{t}\right]=0$ e $|d|<0,5$, vimos que a função densidade espectral de $X_{t}$ pode ser representada por

$$
f_{X}(\lambda)=\left(2 \operatorname{sen}\left(\frac{\lambda}{2}\right)\right)^{-2 d} \frac{\left|\theta\left(e^{-i \lambda}\right)\right|^{2}}{\left|\phi\left(e^{-i \lambda}\right)\right|^{2}} \frac{\sigma_{\epsilon}^{2}}{2 \pi} .
$$

Se $U_{t}=(1-B)^{d} X_{t}$ é um processo $A R M A(p, q)$, então sua função densidade espectral pode ser escrita como

$$
f_{U}(\lambda)=\frac{\left|\theta\left(e^{-i \lambda}\right)\right|^{2}}{\left|\phi\left(e^{-i \lambda}\right)\right|^{2}} \frac{\sigma_{\epsilon}^{2}}{2 \pi}
$$

Logo, temos que

$$
f_{X}(\lambda)=\left(2 \operatorname{sen}\left(\frac{\lambda}{2}\right)\right)^{-2 d} f_{U}(\lambda)
$$

Note que o termo $\left(2 \operatorname{sen}\left(\frac{\lambda}{2}\right)\right)^{-2 d}$ da função densidade espectral está relacionado à propriedade de memória longa da série (parâmetro $d$ ), enquanto que $f_{U}(\lambda)$ está relacionado aos componentes de memória curta (parâmetros AR e MA). Além disso, uma propriedade muito importante de um processo de memória longa é que nas freqüências próximas de zero, a função densidade espectral pode ser aproximada por $f(\lambda) \approx \lambda^{-2 d}$. Um processo de memória curta possui $f(\lambda) \approx 0$ nas baixas freqüências. Portanto, é razóavel obter informações e medidas de memória longa com base no espectro em freqüências muito próximas a zero. Assim, o método proposto por Geweke e Porter-hudak utiliza uma estimativa do espectro, o periodograma $I_{X}(\lambda)$, para a estimação do parâmetro $d$. 
Tome a equação (3.31)

$$
\begin{aligned}
f_{X}(\lambda) & =\left[4 \operatorname{sen}^{2}\left(\frac{\lambda}{2}\right)\right]^{-d} f_{U}(\lambda) \\
& =\frac{f_{U}(0)}{f_{U}(0)}\left[4 \operatorname{sen}^{2}\left(\frac{\lambda}{2}\right)\right]^{-d} f_{U}(\lambda),
\end{aligned}
$$

e tomando o logaritmo em ambos os lados,

$$
\ln \left(f_{X}(\lambda)\right)=\ln \left(f_{U}(0)\right)+(-d) \ln \left(4 \operatorname{sen}^{2}\left(\frac{\lambda}{2}\right)\right)+\ln \left(\frac{f_{U}(\lambda)}{f_{U}(0)}\right) .
$$

Definimos as coordenadas harmônicas por $\lambda=\lambda_{j, T}=\frac{2 \pi j}{T}$. Adicionando $\ln \left[I_{X}\left(\lambda_{j, t}\right)\right]$ em ambos os lados da equação, temos

$\ln \left(I_{X}\left(\lambda_{j, T}\right)\right)=\ln \left(f_{U}(0)\right)+(-d) \ln \left(4 \operatorname{sen}^{2}\left(\frac{\lambda_{j, T}}{2}\right)\right)+\ln \left(\frac{f_{U}\left(\lambda_{j, T}\right)}{f_{U}(0)}\right)+\ln \left(\frac{I_{X}\left(\lambda_{j, T}\right)}{f_{X}\left(\lambda_{j, T}\right)}\right){ }_{3}$

Note que se o termo $\lambda_{j, T}$ assume valores próximos a zero, o termo $\ln \frac{f_{U}\left(\lambda_{j, T}\right)}{f_{U}(0)}$ assume valores desprezíveis. Portanto, selecionamos $j=1,2, \ldots, g(T), g(T)<T$, de forma que $\lambda_{j, T}$ aproxima-se de zero quando $T \rightarrow \infty$. Assim, nas baixas freqüências, o logarítmo do periodograma pode ser aproximado por

$$
\ln \left(I_{X}\left(\lambda_{j, T}\right)\right) \approx \ln \left(f_{U}(0)\right)-d \ln \left(4 \operatorname{sen}^{2}\left(\frac{\lambda_{j, T}}{2}\right)\right)+\ln \left(\frac{I_{X}\left(\lambda_{j, T}\right)}{f_{X}\left(\lambda_{j, T}\right)}\right),
$$

para $j=1,2, \ldots, g(T)$.

Modificando a notação, de forma a facilitar a intepretação dos termos (Geweke e Porter-Hudak, 1993), seja

$$
\begin{aligned}
y_{j} & =\ln \left(I_{X}\left(\lambda_{j, T}\right)\right) \\
x_{j} & =\ln \left[4 \operatorname{sen}^{2}\left(\frac{\lambda_{j, T}}{2}\right)\right], \\
\epsilon_{j} & =\ln \left(\frac{I_{X}\left(\lambda_{j, T}\right)}{f_{X}\left(\lambda_{j, T}\right)}\right)-E\left[\ln \left(\frac{I_{X}\left(\lambda_{j, T}\right)}{f_{X}\left(\lambda_{j, T}\right)}\right)\right], \\
a & =\ln \left(f_{U}(0)\right)+E\left[\ln \left(\frac{I_{X}\left(\lambda_{j, T}\right)}{f_{X}\left(\lambda_{j, T}\right)}\right)\right] \\
b & =-d .
\end{aligned}
$$

Segue-se que a equação (3.34) pode ser representada na forma de um modelo de regressão linear simples dado por

$$
y_{j}=a+b x_{j}+\epsilon_{j}
$$


para $j=1,2, \ldots, g(T)$. O método proposto por Geweke e Porter-Hudak consiste na utilização do método de mínimos quadrados ordinários, para estimar $b=-d$. No entanto, lembremos que uma condição essencial para a validade do modelo é que os termos $\epsilon_{j}$ sejam assintóticamente independentes e com média nula, e o número de pontos utilizados na regressão deve aumentar se aumentarmos o tamanho da série, de forma a garantir a consistência. Assim, escolhemos $g(T)$ de forma que

$$
\lim _{T \rightarrow \infty} \frac{g(T)}{T}=0,
$$

para que sejam analisados somente os valores do periodograma nas baixas freqüências. Além disso, se

$$
\lim _{T \rightarrow \infty} g(T)=\infty
$$

e temos os resultados seguintes,

Proposição 3.1.2. Seja $\left\{X_{t}\right\}$ um processo ARFIMA(p,d,q) com $d<0$. Se $T \rightarrow \infty$, então os termos $\left\{-\ln \left(\frac{I_{X}\left(\lambda_{j, T}\right)}{f_{X}\left(\lambda_{j, T}\right)}\right), j=0,1,2, \ldots, T-1\right\}$, são independentes e seguem uma distribuição de Gumbel com média 0,557216 (constante de Euler) e variância $\frac{\pi^{2}}{6}$.

Proposição 3.1.3. (Chen et al., 1993) Seja $0<d<0,5$ e $f_{X}$ positiva e contínua. Para um inteiro $k>0$ e indices $t_{1}, t_{2}, \ldots, t_{k-1}$, denote o cumulante conjunto de $Z_{t}, Z_{t+t_{1}}, \ldots, Z_{t+t_{k-1}}$ por $c_{k}^{z}\left(t_{1}, \ldots, t_{k-1}\right)$ e assuma que para qualquer $k>0$

$$
\sum_{t_{1}, \ldots, t_{k-1}=-\infty}^{\infty} \mid c_{k}^{z}\left(t_{1}, \ldots, t_{k-1} \mid<\infty .\right.
$$

Considerando $I_{X}\left(\lambda_{j, T}\right)$ um estimador para $f_{X}$, segue de Yajima(1989) que $I_{X}\left(\lambda_{j, T}\right) / f_{X}$ são variáveis assintóticamente $\chi_{2}^{2}$ e independentes.

Utilizando as proposições 3.1.2 e 3.1.3, verifica-se que os termos $\epsilon_{j}=\ln \left(\frac{I_{X}\left(\lambda_{j, T}\right)}{f_{X}\left(\lambda_{j, T}\right)}\right)-$ $E\left[\ln \left(\frac{I_{X}\left(\lambda_{j, T}\right)}{f_{X}\left(\lambda_{j, T}\right)}\right)\right]$ são assintóticamente independentes e com média nula. As funções mais indicadas para $g(T)$ são da forma $g(T)=c T^{\alpha}$, com $0<\alpha<1$.

Proposição 3.1.4. (Geweke e Porter-Hudak, 1983). O estimador do parâmetro de memória longa $\hat{d}_{G P H}$ tem distribuição assintótica normal com média d e variância $\frac{\pi^{2} / 6}{\sum_{i=1}^{g(T)}\left(x_{i}-\bar{x}\right)^{2}}$, onde $g(T)=c T^{\alpha}, 0<\alpha<1$ e $\pi^{2} / 6$ é a variância de $\ln \left(\chi_{2}^{2}\right)$. 
Além disso, Geweke e Porter-Hudak mostraram que pode-se estimar também o desvio padrão de $\hat{d}$ através do desvio padrão estimado do coeficiente de inclinação do modelo de regressão, tornando a implementação do método extremamente simples.

\subsubsection{Estimador usando o Periodograma Suavizado}

Sabe-se que o periodograma é um estimador não consistente para a função densidade espectral $f(\lambda)$. Com o intuito de melhorar o método descrito anteriormente, Chen et al.(1993) e Reisen(1994) sugeriram a utilização de um estimador suavizado, que seja consistente para $f(\lambda)$. Assim, no lugar de (3.34) utilizamos

$$
\ln \left(f_{X}^{T}\left(\lambda_{j, T}\right)\right) \approx \ln \left(f_{U}(0)\right)-d \ln \left(4 \operatorname{sen}^{2}\left(\frac{\lambda_{j, T}}{2}\right)\right)+\ln \left(\frac{f_{X}^{T}\left(\lambda_{j, T}\right)}{f_{X}\left(\lambda_{j, T}\right)}\right),
$$

onde $f_{X}^{T}\left(\lambda_{j, T}\right)$ é o periodograma suavizado, definido por

$$
f_{X}^{T}\left(\lambda_{j, T}\right)=\frac{1}{2 \pi} \sum_{s=-m}^{m} k\left(\frac{s}{m}\right) R(s) \cos \left(s \lambda_{j, T}\right),
$$

$\operatorname{com} \lambda_{j, T}=\frac{2 \pi j}{T}, j=1,2, \ldots, T, k(z)$ uma função par e contínua no intervalo $(-1,1)$ com $k(0)=1$, denominada função geradora da janela espectral e $R(s)$ a função de autocovariância amostral no lag $s$. O parâmetro $m$ é uma função $\mathrm{m}(\mathrm{T})$ de $\mathrm{T}$, de tal forma que

$$
\begin{aligned}
\lim _{T \rightarrow \infty}\left(\frac{m(T)}{T}\right) & =0, \\
\lim _{T \rightarrow \infty} m(T) & =\infty .
\end{aligned}
$$

Usualmente, tomamos $m=T^{\alpha}$, com $0<\alpha<1$.

Diversas janelas podem ser utilizadas para a suavização do periodograma. Porém, as mais utilizadas são as janelas de Bartlett, Daniel, Blackman-Tukey, Hanning, Hamming, Parzen e Bartlett-Priestley (Chen et al., 1993). Veja Priestley (1981) para detalhes e a Tabela 3.1.

Assim como na seção anterior, pode-se escrever um modelo de regressão linear simples, dado por

$$
y_{j}=a+b x_{j}+\epsilon_{j}
$$




\begin{tabular}{|c|c|c|}
\hline Janela Espectral & $k(u)$ & $\alpha=\int_{-1}^{1} k^{2}(u) d u$ \\
\hline Bartlett & $1-|u|$ & $2 / 3$ \\
Daniell & $\operatorname{sen}(\pi u) / \pi u$ & 0,90282336 \\
Blackman-Tukey & $1-2 a+2 a \cos (\pi u)$ & $2\left(1-4 a+6 a^{2}\right)$ \\
Hanning & $0,5+0,5 \cos (\pi u)$ & $0,75(\mathrm{a}=0,25)$ \\
Hamming & $0,54+0,46 \cos (\pi u)$ & $0,7948(\mathrm{a}=0,23)$ \\
Parzen & $\left(1-6 u^{2}+6 u^{3}\right) \operatorname{se}|u| \leq 0,5$ & 0,53928571 \\
& $2(1-|u|)^{3} \operatorname{se} 0,5 \leq|u| \leq 1$ & \\
Bartlett Pristley & $\frac{3}{\pi^{2} u^{2}}\left\{\frac{\sin (\pi u)}{\pi u}-\cos (\pi u)\right\}$ & 1,2 \\
\hline
\end{tabular}

Tabela 3.1: Janelas Espectrais de Suavização

onde

$$
\begin{aligned}
y_{j} & =\ln \left(f_{X}^{T}\left(\lambda_{j, T}\right)\right), \\
x_{j} & =\ln \left[4 \operatorname{sen}^{2}\left(\frac{\lambda_{j, T}}{2}\right)\right] \\
\epsilon_{j} & =\ln \left(\frac{f_{X}^{T}\left(\lambda_{j, T}\right)}{f_{X}\left(\lambda_{j, T}\right)}\right)-E\left[\ln \left(\frac{f_{X}^{T}\left(\lambda_{j, T}\right)}{f_{X}\left(\lambda_{j, T}\right)}\right)\right], \\
a & =\ln \left(f_{U}(0)\right)+E\left[\ln \left(\frac{f_{X}^{T}\left(\lambda_{j, T}\right)}{f_{X}\left(\lambda_{j, T}\right)}\right)\right], \\
b & =-d .
\end{aligned}
$$

Em seguida, estima-se o parâmetro $d$ do modelo de regressão linear simples através do métodos de mínimos quadrados ordinários. O modelo será válido se os termos $\epsilon_{j}$ forem assintóticamente independentes e com média nula, além de que o número de pontos utilizados na regressão deve aumentar se aumentarmos o número de pontos da série. Porém o desvio padrão de $\hat{d}$ não pode mais ser estimado através do desvio padrão estimado do coeficiente de inclinação da reta de regressão.

Priestley(1981) mostrou que $f_{X}^{T}\left(\lambda_{j, T}\right)$ é assintóticamente não viesado, com variância dada por

$$
\lim _{T \rightarrow \infty}\left[\operatorname{Var}\left(f_{X}^{T}(\lambda)\right)\right]= \begin{cases}f_{X}^{2}\left(\frac{m}{T}\right) \int_{-\infty}^{\infty} k(u) d u, & \text { se } \lambda \neq 0, \pi,-\pi \\ 2 f_{X}^{2}\left(\frac{m}{T}\right) \int_{-\infty}^{\infty} k(u) d u, & \text { se } \lambda \in\{0, \pi,-\pi\} .\end{cases}
$$

Proposição 3.1.5. (Anderson, 1971). Seja $f_{X}(\lambda)$ a função densidade espectral de um modelo $\operatorname{ARFIMA}(p, d, q), d \in(-0,5 ; 0)$, e $f_{x}^{T}(\lambda)$ um estimador suavizado, como o descrito 
anteriormente. Então os termos $\ln \left(\frac{f_{X}^{T}\left(\lambda_{j, T}\right)}{f_{X}^{T}\left(\lambda_{j, T}\right)}\right)$ são não-correlacionados com distribuição normal de média zero e variância dada por

$$
\operatorname{Var}\left[\ln \left(\frac{f_{X}^{T}\left(\lambda_{j, T}\right)}{f_{X}\left(\lambda_{j, t}\right)}\right)\right] \approx \begin{cases}\frac{m}{T} \int_{-\infty}^{\infty} k^{2}(u) d u, & \text { se } \lambda \neq 0, \pi,-\pi, \\ \frac{2 m}{T} \int_{-\infty}^{\infty} k^{2}(u) d u, & \text { se } \lambda \in 0, \pi,-\pi .\end{cases}
$$

Proposição 3.1.6. (Chen et al., 1993). Se $d \in(0 ; 0,5)$, o estimador suavizado $f_{X}^{T}(\lambda)$ pode ser aproximado em distribuição por $f_{X}(\lambda) \chi_{\nu}^{2} / \nu$, onde $\nu$ é o correspondente grau de liberdade, dado por

$$
\begin{aligned}
\nu & =\frac{2 T}{m \alpha}, \\
\alpha & =\int_{-1}^{1} k^{2}(u) d u .
\end{aligned}
$$

Note que para as janelas espectrais apresentadas o valor de $\alpha$ pode ser encontrado na Tabela 3.1.

Segundo Chen et al.(1993) os erros $\epsilon_{j}$ do modelo de regressão espectral possui um comportamento de uma variável log-gamma, e conseqüentemente

$$
\begin{array}{r}
E\left(\epsilon_{j}\right) \approx \psi(\nu / 2)-\ln (\nu / 2) \\
\operatorname{Var}\left(\epsilon_{j}\right) \approx \psi^{\prime}(\nu / 2),
\end{array}
$$

onde $\psi=\frac{d}{d x} \ln (\Gamma(x))$ e $\psi^{\prime}=\frac{d^{2}}{d x^{2}} \ln (\Gamma(x))$ são as funções digama e trigama, respectivamente. Verifica-se que os erros $\epsilon_{j}$ não são assintóticamente independentes, mas quando $T \rightarrow$ $\infty$ temos que $m / T \rightarrow 0$ e $\nu \rightarrow \infty$, implicando que $E\left(\epsilon_{j}\right) \rightarrow 0$ e $\operatorname{Var}\left(\epsilon_{j}\right) \rightarrow 0$, ou seja, uma distribuição degenerada, e conseqüentemente o estimador suavizado de $d$ possui assintóticamente uma distribuição degenerada.

Chen et al.(1993) mostraram que o estimador suavizado $\hat{d}_{S}$ é assintóticamente normal,

$$
\hat{d}_{S} \sim N\left(d, \frac{\psi^{\prime}(\nu / 2)}{\sum_{j=1}^{g(T)}\left(x_{i}-\bar{x}\right)^{2}}\right),
$$

onde $g(T)$ é da forma $g(T)=c T^{\xi}$, com $0<\xi<1$ e $c$ constante.

Reisen(1994) em um estudo completamente independente, mostrou que a distribuição 
assintótica de $\hat{d}_{S}$ também pode ser expressa na forma

$$
\hat{d}_{S} \sim N\left(d, \frac{m \int_{-1}^{1} k(u)^{2} d u}{\sum_{j=1}^{g(T)}\left(x_{i}-\bar{x}\right)^{2}}\right) .
$$

Reisen(1994) e Chen et al.(1993) mostraram através de simulações que a estimação do parâmetro $d$ utilizando o periodograma suavizado é melhor que utilizando o periodograma bruto, em relação a varibilidade e erro quadrático médio. No entanto Chen et al.(1993) verificaram que para séries finitas os estimadores suavizados possuem um ligeiro viés. Além disso, Chen et al.(1993) mostraram que as janelas espectrais com melhor perfomance são as janelas de Daniel e Bartlett-Priestley.

\subsubsection{Estimador Truncado de Whittle}

Este estimador foi proposto por Robinson (1995) e baseia-se também na estimação semiparamétrica do parâmetro $d$, assim como o estimador de Geweke e Porter-Hudak. No entanto, verifica-se que é assintóticamente mais eficiente e mais robusto em determinadas situações.

O método considera a aproximação de Whittle(1953) para o negativo da função de verossimilhança de um modelo $\operatorname{ARFIMA}(p, d, q)$ gaussiano, dada por

$$
L=\frac{1}{\pi}\left[\sum_{j=1}^{m} \frac{2 \pi I\left(\lambda_{j}\right)}{I\left(T \lambda_{j}\right)}+\sum_{j=1}^{m} \log \left(I\left(\lambda_{j}\right)\right) \frac{2 \pi}{T}\right] .
$$

Como nas baixas freqüências a função densidade espectral de um processo de memória longa pode ser aproximado por $c \lambda_{j}^{-2 d}$, o negativo da função de verossimilhança aproximada é

$$
L \approx \frac{1}{m}\left[\sum_{j=1}^{m} \frac{I\left(\lambda_{j}\right)}{c \lambda_{j}^{-2 d}}+\sum_{j=1}^{m} \log \left(c \lambda_{j}^{-2 d}\right)\right] .
$$

Minimizando a função e relação a $c$, uma estimativa para o parâmetro de memória longa é o valor de $d$ que minimiza a quantidade

$$
\log \left(\frac{1}{m} \sum_{j=1}^{m} \frac{I\left(\lambda_{j}\right)}{\lambda_{j}^{-2 d}}\right)+\frac{2 d}{m} \sum_{j=1}^{m} \log \left(\lambda_{j}\right),
$$


onde $m=g(T)$, e deve satisfazer a condição

$$
\frac{1}{m}+\frac{m}{T} \rightarrow 0
$$

quando $T \rightarrow \infty$. Note que na realidade, o estimador Truncado de Whittle $d_{t w}$ é o valor $d$ que maximiza uma função aproximada da verossimilhança.

Além de ser teoricamente mais eficiente que o estimador GPH, Velasco (1999) mostrou que, sob determinadas condições, o estimador é consistente para $d \in(-0,5 ; 1)$ e assintóticamente normal para $d \in(-0,5 ; 0,75)$. Robinson e Henry(1999) mostraram também que o limite em distribuição deste estimador não é afetado por heterocedasticidade.

\subsubsection{Modelo de Volatilidade Estocástica de Memória Longa}

Recentemente verificou-se que a volatilidade de diversos ativos financeiros são processos que apresentam memória longa (Ding, Granger e Engle, 1993). Um dos modelos mais populares nesta situção é o modelo de volatilidade estocástica proposto por Breidt, Crato e de Lima (1998). O modelo de volatilidade estocástica simples já foi descrito no capítulo 2 e o modelo de volatilidade estocástica de memória longa é apenas uma generalização. É definido por

$$
\begin{array}{r}
r_{t}=\sigma_{t} \xi_{t}, \\
\sigma_{t}=\sigma \exp \left\{\frac{x_{t}}{2}\right\},
\end{array}
$$

onde $r_{t}$ é o retorno simples ou log-retorno do ativo de interesse, $x_{t}$ é um processo de memória longa com média nula e independente do ruído branco $\xi_{t}$. Note que tomando o logarítmo dos quadrados dos retornos, temos

$$
\begin{aligned}
\ln \left(r_{t}^{2}\right) & =\ln \left(\sigma^{2}\right)+x_{t}+\ln \left(\xi_{t}\right) \\
& =\ln \left(\sigma^{2}\right)+E\left[\ln \left(\xi_{t}^{2}\right)\right]+x_{t}+\left(\ln \left(\xi_{t}^{2}\right)-E\left[\ln \left(\xi_{t}^{2} x_{t}\right)\right]\right) \\
& =c+x_{t}+\epsilon_{t} .
\end{aligned}
$$

Portanto, podemos escrever $\ln \left(r_{t}^{2}\right)$ como a soma de uma constante, um processo de memória longa e um processo de memória curta com média nula. No entanto, verifica-se que se assumirmos que $\xi_{t}$ segue uma distribuição gaussiana, o termo de memória curta 
$\epsilon_{t}$ possui distribuição qui-quadrado. Veremos mais tarde que esta representação é extremamente útil para a identificação de processos de memória longa compartilhada, tema do próximo capítulo. 
CAPÍTULO 4

\section{Memória Longa Compartilhada}

Neste capítulo serão apresentados conceitos e métodos relativos a processos de memória longa compartilhada em séries temporais, com enfoque no método de identificação proposto por Ray e Tsay(1997) através da análise de correlação canônica, cujo desempenho será avaliado no capítulo 5 .

\subsection{Processos com Memória Longa Compartilhada}

Freqüentemente dados de séries temporais são observados simultaneamente em um intervalo de tempo, ou seja, é possível observar $k$ séries temporais no mesmo instante, tais como a temperatura, velocidade do vento e índice de umidade em um determinado local. Com o avanço computacional dos últimos anos, as análises multivariadas estão sujeitas a um maior refinamento, de forma que o estudo de relações entre séries simultâneas vem atraindo a atenção de pesquisadores da área de econometria, climatologia e hidrologia, principalmente na tentativa de identificar componentes comuns às séries.

Engle e Granger(1987), Stock e Watson(1998) e Johansen(1991) introduziram o conceito de séries co-integradas, onde é possível encontrar combinações lineares estacionárias de séries não-estacionárias. Em termos práticos, verifica-se que séries co-integradas são séries que apresentam tendências semelhantes, ou seja, parecem "flutuar" sobre uma mesma onda. Embora este conceito de co-integração tenha sido especificado para séries não-estacionárias com parâmetro de integração $d=1$, o conceito de co-integração fracionária foi proposto por Cheung e Lai (1993). Veremos mais tarde que a memória longa 
compartilhada é um caso particular de co-integração fracionária.

Além disso, diversos estudos mostraram a aplicabilidade da análise de correlação canônica (Hottelling, 1936) na modelagem de séries temporais. Box e Tiao (1977) propuseram um método de decomposição de séries estacionárias k-dimensionais em k componentes ortogonais ordenados do menos previsível ao mais previsível. Verifica-se, em geral, que a série mais previsível é não-estacionária e representa a dinâmica de crescimento das séries, enquanto que a série menos previsível é estacionária. Tsay e Tiao (1985), Tsay (1987) e Tiao e Tsay (1989) estudaram diversos métodos de identificação de estruturas de processos ARMA multivariados, visando a construção de modelos parcimoniosos. Na realidade, verifica-se que os métodos em séries temporais que utilizam análise de correlação canônica visam obter combinações lineares com características peculiares e que, em grande parte dos casos, não herdam a estrutura das séries originais. O teste para memória longa compartilhada (MLC), proposto por Ray e Tsay (1997), verifica se existem combinações lineares de séries com memória longa, onde as combinações não apresentam persistência, ou seja, possuem memória curta.

Exemplificando um modelo de memória longa compartilhada, considere um caso bivariado tal que

$$
\begin{aligned}
\mathbf{y}_{t} & =\left(y_{1 t}, y_{2 t}\right)^{\prime}, \\
y_{1 t} & =\alpha x_{t}+w_{1 t}, \\
y_{2 t} & =x_{t}+w_{2 t},
\end{aligned}
$$

onde $\alpha>0, x_{t}$ é um processo memória longa de ordem $d \in(0 ; 0,5)$, e $w_{1 t}$ e $w_{2 t}$ são processos de memória curta possivelmente correlacionados. Note que o processo $x_{t}$ é comum para as duas séries, de forma que $y_{1 t}$ e $y_{2 t}$ só possuem memória longa devido a este termo. Além disso, é fácil verificar que $\gamma_{y_{1}}(h)=\gamma_{x}(h)$ e $\gamma_{y_{2}}(h)=\alpha^{2} \gamma_{x}(h)$ para valores de $h$ suficientemente grandes, pois $\gamma_{w_{1}}(h) \rightarrow 0$ e $\gamma_{w_{2}}(h) \rightarrow 0$. Portanto, para h grande, a função de autocovariância de $y_{1 t}$ e $y_{2 t}$ são proporcionais, implicando que a matriz de correlação cruzada das séries não possui posto completo. Este fato é a chave do teste de memória longa compartilhada. Considere novamente o modelo (4.1). Note que $y_{i t}$ possui memória longa, mas existe um vetor $\mathbf{v}=(1,-\alpha)^{\prime}$ de tal forma que

$$
\begin{aligned}
y_{t}^{*} & =\mathbf{v}^{\prime} \mathbf{y}_{t}=y_{1 t}-\alpha y_{2 t} \\
& =w_{1 t}-\alpha w_{2 t},
\end{aligned}
$$


ou seja, $y_{t}^{*}=\mathbf{v}^{\prime} \mathbf{y}_{t}$ é um processo de memória curta, pois é combinação linear somente dos $w_{i t}^{\prime} s$. Como o termo $x_{t}$ é comum às duas séries, dizemos que $y_{1 t}$ e $y_{2 t}$ possuem memória longa compartilhada. Este conceito pode ser generalizado para $k$ séries que possuem $s$ combinações lineares distintas de $y_{i t}$ que são processos de memória curta.

Um fato importante a ser levado em conta é que, embora a condição de que as séries $y_{i t}$ possuam o mesmo parâmetro de memória longa $d$ seja necessária, ela não é suficiente para garantir que as séries possuam componentes comuns de memória longa, caraterística fundamental existente em processos com memória longa compartilhada.

\subsection{Teste de Correlação Canônica para MLC}

A forma geral de um modelo com memória longa compartilhada (Ray e Tsay, 1997) é dada por

$$
\mathbf{y}_{t}=\mathbf{C x}_{t}+\mathbf{w}_{t}
$$

onde $\mathbf{y}_{t}$ e $\mathbf{w}_{t}$ possuem dimensão $k, \mathbf{x}_{t}$ é um processo de memória longa $r$-dimensional $(0<r<k)$ e $\mathbf{C}$ é uma matriz de coeficientes de ordem $k \times r$. As séries $\mathbf{x}_{t}$ e $\mathbf{w}_{t}$ são independentes, e $\mathbf{w}_{t}$ é um processo de memória curta.

Considere $\boldsymbol{\Gamma}_{u}(l)$ a matriz de autocovariância no lag $l$ de uma série $\mathbf{u}_{t}$ multivariada, definida por

$$
\boldsymbol{\Gamma}_{\mathbf{u}}(l)=\left(\begin{array}{cccc}
\gamma_{u_{1}}(l) & \gamma_{u_{1} u_{2}}(l) & \ldots & \gamma_{u_{1} u_{k}}(l) \\
\gamma_{u_{2} u_{1}}(l) & \gamma_{u_{2}}(l) & \ldots & \gamma_{u_{2} u_{k}}(l) \\
\vdots & \ddots & \ldots & \vdots \\
\gamma_{u_{k} u_{1}}(l) & \gamma_{u_{k} u_{2}}(l) & \ldots & \gamma_{u_{k}}(l)
\end{array}\right)
$$

onde $\gamma_{u_{k}}(l)$ é função de autocovariância no lag $l$ da série univariada $u_{k, t}$ e $\gamma_{u_{j} u_{m}}(l)$ é função de autocovariância cruzada no lag $l$ das série $u_{j, t}$ e $u_{m, t}$.

Assumindo que as séries do modelo (4.2) são estacionárias, temos que

$$
\boldsymbol{\Gamma}_{y}(l)=\mathbf{C} \boldsymbol{\Gamma}_{x}(l) \mathbf{C}^{\prime}+\boldsymbol{\Gamma}_{w}(l) .
$$

Verifica-se portanto, que a função de autocovariância de $\mathbf{y}_{t}$ é soma das funções de autocovariância do processo de memória longa com o processo de memória curta. Lem- 
bremos que a f.a.c. de processos de memória longa decai em velocidade hiperbólica para zero, enquanto que a f.a.c. de processos de memória curta decai exponencialmente. Como $w_{t}$ possui memória curta, temos que, quando $l \rightarrow \infty$,

$$
\begin{aligned}
& \boldsymbol{\Gamma}_{w}(l) \rightarrow_{e} 0, \\
& \boldsymbol{\Gamma}_{y}(l) \rightarrow_{e} \mathbf{C} \boldsymbol{\Gamma}_{x}(l) \mathbf{C}^{\prime},
\end{aligned}
$$

onde $\rightarrow_{e}$ denota convergência em taxa exponencial. Particularmente, se $\mathbf{w}_{t}$ é um processo $\operatorname{MA}(q)$

$$
\boldsymbol{\Gamma}_{y}(l)=\mathbf{C} \boldsymbol{\Gamma}_{x}(l) \mathbf{C}^{\prime}, \quad l>q .
$$

Note que para $l$ grande, se $\mathbf{C}$ possui posto completo, o posto de $\boldsymbol{\Gamma}_{y}(l)=\mathbf{C} \boldsymbol{\Gamma}_{x}(l) \mathbf{C}^{\prime}$ é o mesmo de $\boldsymbol{\Gamma}_{x}(l)$, ou seja $r$. Como $r<k$ e $\boldsymbol{\Gamma}_{y}(l)$ é uma matriz de ordem $k \times k, \boldsymbol{\Gamma}_{y}(l)$ não possui posto completo.

Para verificarmos essas propriedades para mais de um lag, considere $h>0$ e $j>$ 0 , seja o vetor $\mathbf{Y}_{h, j, t}=\left(\mathbf{y}_{t-j}, \mathbf{y}_{t-j-1}, \ldots, \mathbf{y}_{t-j-h+1}\right)^{\prime}$ uma coleção de $h$ valores passados do sistema após $j$ defasagens. A matriz de covariância entre $\mathbf{y}_{t}$ e $\mathbf{Y}_{h, j, t}$ é dada por $\mathbf{G}(h, j)=$ $\left[\boldsymbol{\Gamma}_{y}(j), \ldots, \boldsymbol{\Gamma}_{y}(j+h-1)\right]$, uma matriz de ordem $k \times h k$. Para o modelo (4.2), utilizando as equações (4.3), (4.4) e (4.5), temos

$$
\mathbf{G}(h, j) \rightarrow_{e} \mathbf{C}\left[\boldsymbol{\Gamma}_{x}(j), \boldsymbol{\Gamma}_{x}(j+1), \ldots, \boldsymbol{\Gamma}_{x}(j+h-1)\right] \mathbf{C}^{\prime},
$$

para $h \geq 1$ e $j<\infty$ suficientemente grande. Note que, como as séries possuem memória longa compartilhada, $\boldsymbol{\Gamma}_{x}(j)$ não possui posto completo, pois é uma matriz $k \times k$ e só temos $r<k$ processos de memória longa. Dessa forma, é fácil concluir que matriz $\mathbf{G}(h, j)$ não possui posto completo. Conseqüentemente, uma forma de identificação da estrutura do modelo (4.2) é a análise o posto de $\mathbf{G}(h, j)$ para $j$ suficientemente grande.

Segundo Tsay e Tiao (1985), Tsay (1987) e Tiao e Tsay (1989), uma forma conveniente de se verificar o posto da matriz $\mathbf{G}(h, j)$ é considerar as correlações canônicas existentes entre $\mathbf{y}_{t}$ e $\mathbf{Y}_{h, j, t}$. Temos que se $\mathbf{G}(h, j)$ possui posto $r$ então existem $s=k-r$ correlações canônicas entre $\mathbf{y}_{t}$ e $\mathbf{Y}_{h, j, t}$ que são iguais a zero. Na análise estatística multivariada, a análise de correlação canônica é utilizada para encontrar combinações lineares ortogonais que maximizam a correlação entre variáveis $(\mathrm{U}, \mathrm{V})$. No entanto, é importante ressaltar que no caso do teste MLC, a análise de correlação canônica é utilizada para testar o posto de matrizes, verificando a significância de seus autovalores. 
Proposição 4.2.1. (Ray e Tsay, 1997). Seja $\mathbf{y}_{t}$ uma série estacionária k-dimensional com memória longa compartilhada e $h$ número inteiro positivo. Então existem $k-r$ correlações canônicas entre $\mathbf{y}_{t}$ e $\mathbf{Y}_{h, j, t}$ que convergem para zero, quando $j \rightarrow \infty$. Além disso, se $\mathbf{w}_{t}$ é um processo $M A$ de ordem $q$, então existem $s=k-r$ correlações canônicas entre $\mathbf{y}_{t}$ e $\mathbf{Y}_{h, j, t}$ para todo $j>q$.

Dados $h$ e $j$, o quadrado das correlações canônicas entre $\mathbf{y}_{t}$ e $\mathbf{Y}_{h, j, t}$ são os autovalores da matriz

$$
\mathbf{A}(h, j)=\left[\operatorname{Var}\left(\mathbf{y}_{t}\right)\right]^{-1} \operatorname{Cov}\left(\mathbf{Y}_{h, j, t}, \mathbf{y}_{t}\right)\left[\operatorname{Var}\left(\mathbf{Y}_{h, j, t}\right)\right]^{-1} \operatorname{Cov}\left(\mathbf{y}_{t}, \mathbf{Y}_{h, j, t}\right) .
$$

Denote os autovalores de $\mathbf{A}(h, j)$ por $0 \leq \rho_{1}^{2} \leq \rho_{2}^{2} \leq \ldots \rho_{k}^{2} \leq 1$. Note que o posto da matriz $\mathbf{A}(h, j)$ é o mesmo de $\mathbf{G}(h, j)=\operatorname{Cov}\left(\mathbf{Y}_{h, j, t}, \mathbf{y}_{t}\right)$, pois $\operatorname{Var}\left(\mathbf{y}_{t}\right)$ e $\operatorname{Var}\left(\mathbf{Y}_{h, j, t}\right)$ possuem posto completo. O posto de $\mathbf{A}(h, j)$ é $(k-s)$, onde s é o número de autovalores iguais a zero. Para testar o posto da matriz $\mathbf{A}(h, j)$, devemos testar as hipóteses

$$
H_{S O}: \rho_{s}^{2}=0 \quad \text { vs } \quad H_{S A}: \rho_{s}^{2}>0,
$$

para $\mathrm{s}=1, \ldots, \mathrm{k}$.

Um estimador para a matriz $\mathbf{A}(h, j)$ é

$$
\hat{\mathbf{A}}(h, j)=\hat{\Sigma}_{y y}^{-1} \hat{\Sigma}_{y Y} \hat{\Sigma}_{Y Y}^{-1} \hat{\Sigma}_{Y y},
$$

onde

$$
\begin{aligned}
\hat{\Sigma}_{y y} & =\sum\left(\mathbf{y}_{t}-\hat{\mu}_{y}\right)\left(\mathbf{y}_{t}-\hat{\mu}_{y}\right)^{\prime} / n, \\
\hat{\Sigma}_{y Y} & =\sum\left(\mathbf{y}_{t}-\hat{\mu}_{y}\right)\left(\mathbf{Y}_{h, j, t}-\hat{\mu}_{Y}\right)^{\prime} / n, \\
\hat{\Sigma}_{Y Y} & =\sum\left(\mathbf{Y}_{h, j, t}-\hat{\mu}_{Y}\right)\left(\mathbf{Y}_{h, j, t}-\hat{\mu}_{Y}\right)^{\prime} / n, \\
\hat{\Sigma}_{y Y} & =\hat{\Sigma}_{Y y}^{\prime},
\end{aligned}
$$

e $\hat{\mu}_{y}, \hat{\mu}_{Y}$ são as médias amostrais de $\mathbf{y}_{t}$ e $\mathbf{Y}_{h, j, t}$ respectivamente.

Para testar o posto de $\mathbf{A}(h, j)$, considere a seguinte estatística:

$$
T_{s}=-(n-h) \sum_{i=1}^{s} \ln \left(1-\hat{\rho}_{i}^{2}\right),
$$

onde $0 \leq \hat{\rho}_{1}^{2} \leq \hat{\rho}_{2}^{2} \leq \ldots \hat{\rho}_{k}^{2} \leq 1$ são os autovalores da matriz $\hat{\mathbf{A}}(h, j)$. 
Proposição 4.2.2. (Ray e Tsay, 1997). Seja processo estacionário de memória longa $\mathbf{y}_{\boldsymbol{t}}$ descrito por (4.2.1), e $y_{i t}^{*}$ variável canônica de $\mathbf{y}_{t}$ correspondente às s menores correlações canônicas entre $\mathbf{y}_{t}$ e $\mathbf{Y}_{h, j, t}$ não correlacionadas serialmente, onde $h$ e $j$ são inteiros positivos. Então a estatística de teste $T_{s}$ tem distribuição assintótica qui-quadrado com $s[(h-1) k+s]$ graus de liberdade, sob a hipótese nula $H_{S O}$ em (4.9).

Se $\mathbf{v}_{i}$ é o autovetor correpondente ao autovalor $\hat{\rho}_{i}$ da matriz $\hat{\mathbf{A}}(h, j)$, temos que $y_{i t}^{*}=\mathbf{v}_{i}^{\prime} \mathbf{y}_{t}$ é a variável canônica relacionada a $\hat{\rho}_{i}$. Uma das suposições do teste descrito anteriormente é que as variáveis canônicas são não-correlacionadas serialmente, mas essa suposição muitas vezes não é válida em aplicações práticas. Portanto Ray e Tsay(1997) sugeriram a utilização da estatística modificada

$$
T_{s}^{*}=-(n-h) \sum_{i=1}^{s} \ln \left(1-\frac{\hat{\rho}_{i}^{2}}{g_{i}}\right),
$$

onde $g_{i}=\left(1+2 \sum_{u=1}^{j-1} \hat{\rho}_{r, u} \hat{\rho}_{l, u}\right)$ é um fator de correção. Temos que $\hat{\rho}_{r, u}$ e $\hat{\rho}_{l, u}$ são os valores da função de autocorrelação amostral no lag $u$ das variáveis canônicas $y_{i t}^{*}$ e $Y_{i t}^{*}$ respectivamente. $Y_{i t}^{*}$ é definido por $Y_{i t}^{*}=V_{i}^{\prime} Y_{h, j, t}$ onde $V_{i}$ é o autovetor associado ao autovalor $\hat{\rho}_{i}^{2}$ da matriz de ordem $h k \times h k$, definida por

$$
\hat{\mathbf{D}}(h, j)=\hat{\Sigma}_{Y Y}^{-1} \hat{\Sigma}_{Y y} \hat{\Sigma}_{y y}^{-1} \hat{\Sigma}_{y Y} .
$$

Como $\hat{\mathbf{A}}(h, j)$ ignora $j$ valores passados de $\mathbf{y}_{t}$, é razoável supor que $y_{i t}^{*}$ segue um processo $\mathrm{MA}(j-1)$. A fórmula de Barlett para a variância da correlação cruzada amostral entre duas séries temporais pode ser utilizada para derivar a variância assintótica de $\hat{\rho}_{i}$.

Proposição 4.2.3. (Ray e Tsay, 1997). Sejam $h, j \geq 1, \mathbf{y}_{t}$ satisfaz as condições descritas na proposição 4.2.1, e as variáveis canônicas $y_{i t}^{*}$ seguindo um processo $M A(j-1)$. Então a estatística de teste $T_{s}^{*}$ possui assintóticamente uma distribuição qui-quadrado com s[(h$1) k+s]$ graus de liberdade sob a hipótese nula $H_{S 0}$.

Tendo em vista os aspectos apresentados, verifica-se que dois parâmetros necessitam ser especificados para a realização do teste MLC: o parâmetro de defasagem $j$ e o número de autocovariâncias $h$ a serem utilizadas.O parâmetro j deve ser escolhido conforme as suposições feitas para a estrutura de autocorrelação dos componentes de memória curta de $y_{i t}, i=1, \ldots, k$. Na verdade, $j$ deveria ser suficientemente grande, de forma que todas as 
variáveis canônicas $y_{i t}^{*}$ sejam não correlacionadas após o lag $j$, lembrando que um processo $M A(q)$ apresenta autocorrelação nula após o lag $q$ e que a f.a.c. de um processo $A R(p)$ decai exponencialmente, podendo ser aproximado por um processo $M A$ de ordem grande. Porém, se um $j$ muito grande for escolhido, mesmo que as séries possuam componentes de memória longa, ela pode não ser detectada, invalidando o teste estatístico. O parâmetro $\mathrm{h}$ deve ser escolhido de tal forma que $\mathbf{Y}_{h, j, t}$ represente adequadamente a dinâmica existente entre as componentes de memória curta do processo $\mathbf{y}_{t}$. Na prática, os valores $j$ e $h$ devem ser escolhidos de acordo com os tamanhos das séries, e informações sobre estudos prévios realizados com as séries em questão. 
CAPÍTULO 5

\section{Simulações}

Um dos principais objetivos deste trabalho é a avaliação do comportamento de estimadores tradicionais do parâmetro de memória longa " d" para processos mistos, ou seja, para processos de memória longa adicionados à processos de memória curta. Esta situação é coerente com modelos de Memória Longa Compartilhada(MLC) vistos no capítulo anterior. Um segundo objetivo é o estudo do desempenho e comportamento do teste $M L C$ propostos por Ray e Tsay(1997) para processos de memória longa $A R F I M A(p, d, q)$ gaussianos adicionados à processsos de memória curta com distribuição gaussiana e qui-quadrado.

Neste capítulo serão apresentados os resultados de um conjunto de nove simulações realizadas. Os conjuntos de 1 a 4 (Apêndice A) são referentes aos estimadores do parâmetro de memória longa e os conjuntos 5 ao 7 (Apêndice B) referentes ao teste MLC. Todas as rotinas para estimação, testes e simulação foram implementadas para o software R.

\subsection{Avaliação de Estimadores}

Os estimadores do parâmetro fracionário a serem avaliados são três: o estimador de Geweke e Porter-Hudak $\left(d_{G P H}\right)$, o estimador do periodograma suavizado $\left(d_{S}\right)$ e o estimador semiparamétrico truncado de Whittle $\left(d_{T W}\right)$. Devido a sua simplicidade, o estimador de Geweke e Porter Hudak é um dos mais populares e freqüentemente utilizados. No entanto estudos prévios mostram que embora seja não-viesado, possui pouco poder para testar as hipóteses $H_{0}: d=0$ vs $H_{A}: d \neq 0$, pois apresenta grande variabilidade. Utilizando-se o periodograma suavizado, ou seja, uma estimativa assintóticamente consistente para a 
função espectral, obtemos um estimador com menor variabilidade, porém viesado para amostras finitas (Chen et al., 1993). O estimador Truncado de Whittle foi proposto por Robinson(1994) e baseia-se na aproximação de Whittle para a verossimilhança e atualmente está se tornando popular na literatura, devido sua robustez no caso não-estacionário (Velasco, 1999) e heterocedasticidade(Robinson e Henry, 1999).

A função do tamanho da série $(T)$, que especifica o número de pontos a serem utilizados na regressão espectral (para os estimadores $d_{G P H}$ e $d_{S}$ ) é dada por $g(T)=T^{0.5}$. A função de especificação do número de pontos a serem utilizados na janela espectral de suavização é $m(T)=T^{0.9}$. Segundo Moura(1995) tal parametrização apresenta bons resultados para os estimadores que utilizam o método da regressão espectral. A janela espectral de suavização utilizada foi a janela de Bartlett-Priestley dada por $k(u)=\frac{3}{\pi^{2} u^{2}}\left\{\frac{\sin (\pi u)}{\pi u}-\cos (\pi u)\right\}$, que segundo Chen et al.(1993) é a que apresenta o melhor desempenho. Para o estimador truncado de Whittle, o parâmetro de truncamento é dado por $g(T)=T^{0.7}$, pois através de simulações prévias, verificou-se que apresenta bons resultados em relação ao viés e à variabilidade.

\subsubsection{Conjunto 1: Processos ARMA}

O objetivo deste primeiro conjunto de simulações é avaliar o comportamento dos três estimadores de memória longa em processos $\operatorname{ARFIMA}(1,0,1)$, em relação a variações nos coeficientes autoregressivos $\phi$ e de médias móveis $\theta$. Sabe-se que quanto maior o valor de $|\phi|$ mais lento é o decaimento para zero da função de autocorrelação, embora em taxa exponencial. Portanto, é razoável avaliar se existe alguma relação entre o coeficiente $\phi$ e os estimadores dos parâmetros de memória longa. Além disso, também desejamos verificar se o coeficiente $\theta$ possui algum efeito nos estimadores, pois a função de autocorrelação parcial também decai mais lentamente, conforme cresce o valor de $\theta$.

Os processos $y_{t}$ simulados são da forma:

$$
\phi(B) y_{t}=\theta(B) \epsilon_{t}
$$

onde $\epsilon_{t}$ é um ruído branco gaussiano.

Foram simuladas séries de tamanho $T=500$ (Tabela A.1) e $T=1000$ (Tabela A.2) para verificar o efeito do tamanho da série nos estimadores. Os coeficientes $\theta$ e $\phi$ estão no 
intervalo $[-0,99 ; 0,99]$, mas seus efeitos foram analisados separadamente, ou seja, se $\theta \neq 0$ então $\phi=0$ e vice-versa.

\section{Conclusões}

Como esperado, a estimação utilizando séries maiores $(T=1000)$ apresenta sempre menor variabilidade, viés e erro quadrático médio do que séries com menor tamanho $(T=500)$. Além disso, verifica-se que valores negativos dos coeficientes autoregressivos e médias móveis praticamente não afetam os estimadores de $d$. Porém para coeficientes positivos o módulo do viés aumenta gradualmente para os três estimadores.

Neste caso, o estimador de Geweke e Porter-Hudak apresenta pouco viés, mas maior variabilidade. O método do periodograma suavizado apresenta ligeiro viés negativo mas possui menor variabilidade, de forma que possui menor erro quadrático médio. Verifica-se que o estimador truncado de Whittle $\left(\hat{d}_{T W}\right)$ é o que apresenta a menor variância, porém é o mais afetado por variações nos coeficientes, de forma que se torna altamente viesado, para valores positivos dos coeficientes $\phi$ e $\theta$. Devido à baixa variabilidade do estimador $\hat{d}_{T W}$, o teste utilizando-o para testar $H_{0}: d=0$ vs $H_{A}: d \neq 0$ é o mais poderoso. Porém, devido ao viés, apresenta uma baixa cobertura quando a hipótese nula é verdadeira e temos coeficientes $\phi$ e $\theta$ positivos, conseqüentemente, rejeita-se em demasiado a hipótese nula, mesmo que verdadeira. Para cada simulação é possível contruir um intervalo de confiança de $\gamma \%$ para o parâmetro $d$. A cobertura de um estimador é dada pela proporção de vezes em que o verdadeiro valor da média está contido dentro deste intervalo. As Figuras 5.1 e 5.3 apresentam o viés e as Figuras 5.2 e 5.4 o erro quadrático médio dos estimadores, para variações em $\phi$ e $\theta$ para $T=1000$.

\subsubsection{Conjunto 2: Razão de Desvios e Memória Longa}

Neste segundo conjunto de simulações, deseja-se a avaliar o comportamento dos estimadores de memória longa em relação à razão dos desvios para diversos valores de $d$. A razão de desvios(RD) é definida como a razão entre o desvio padrão do termo de memória curta e do termo de memória longa. Os modelos simulados são da forma:

$$
y_{t}=x_{t}+\lambda w_{t}
$$




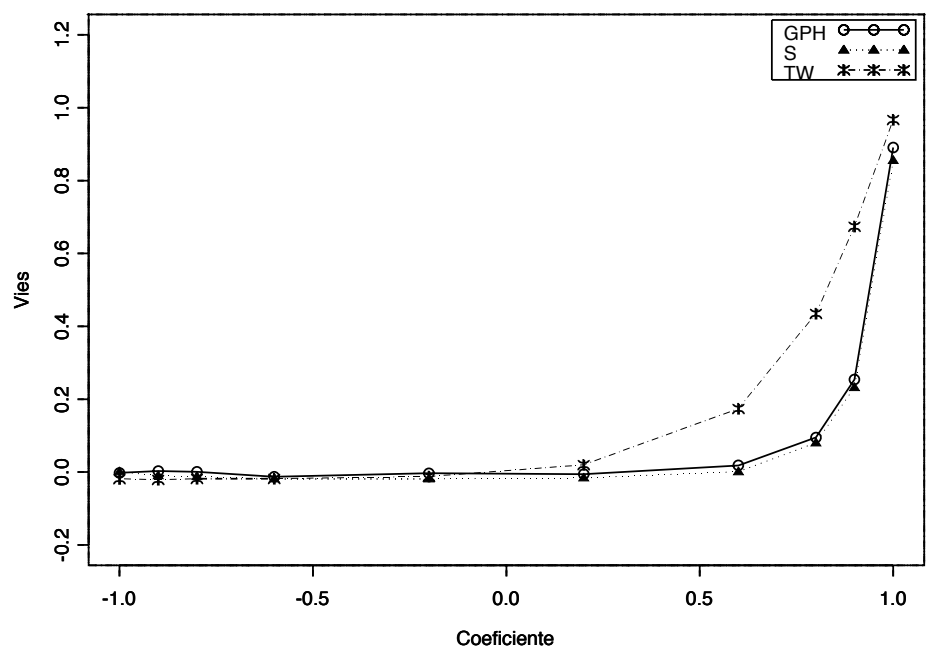

Figura 5.1: Viés dos Estimadores para variações em $\phi$ quando $d=0$.

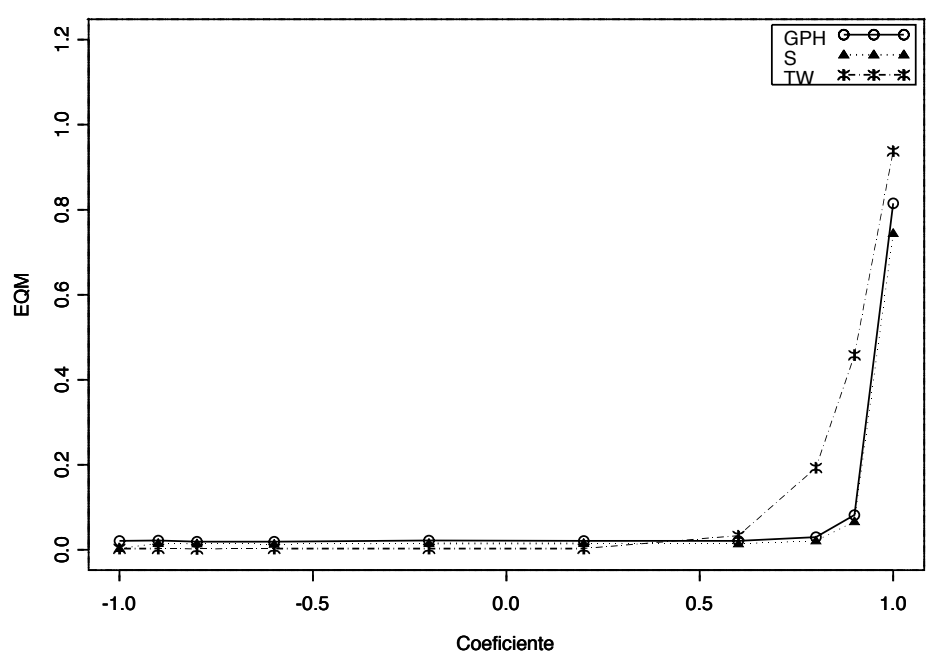

Figura 5.2: Erro Quadrático Médio dos Estimadores para variações em $\phi$ quando $d=0$. 


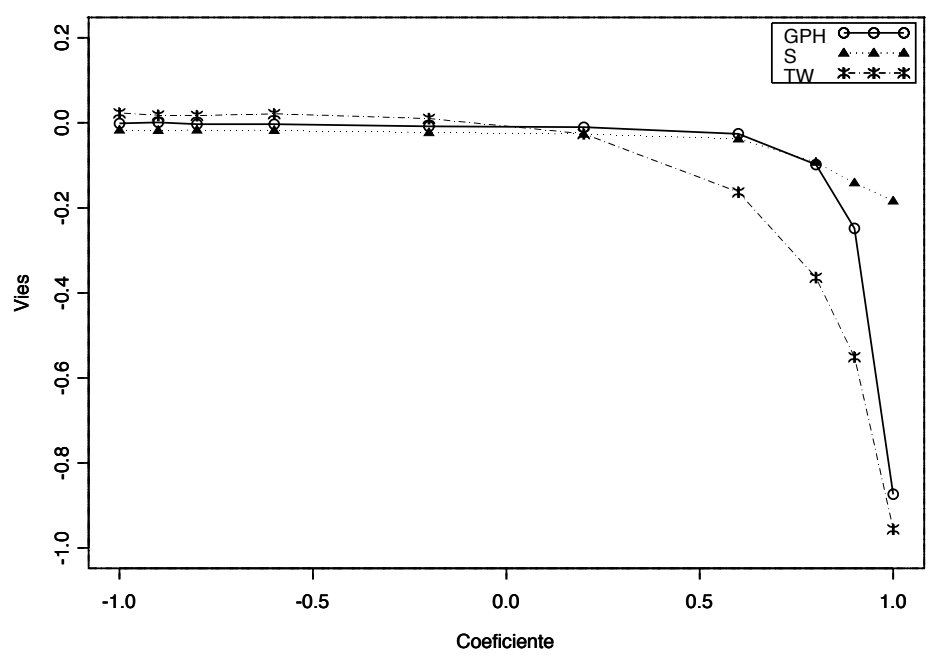

Figura 5.3: Viés dos Estimadores para variações em $\theta$ quando $d=0$.

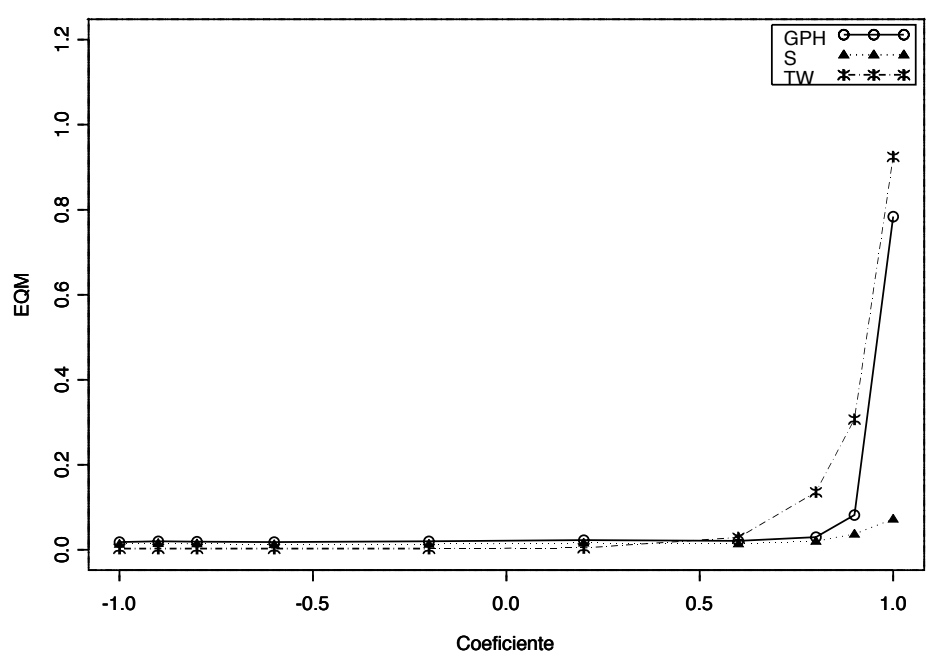

Figura 5.4: Erro Quadrático Médio dos Estimadores para variações em $\theta$ quando $d=0$. 
onde $\lambda$ é uma constante, $x_{t}$ é um ruído branco fracionário e $w_{t}$ é um ruído branco. Em outras palavras, o processo consiste na soma de uma parcela de memória longa com uma parcela de memória curta. Espera-se que quanto maior a variância do processo de memória curta, maior será o viés na estimação de $d$. Nas simulações realizadas os processos $x_{t} \mathrm{e}$ $w_{t}$ possuem o desvio padrão unitário, portanto, é fácil ver que $R D=\lambda$. Os valores de $d$ das simulações são $0,15,0,30$ e 0,45 , e os valores de $R D$ são $0,0,6,1,0$ e 2,0. Além disso, também verificou-se o desempenho dos estimadores assumindo $w_{t}$ com distribuição gaussiana (Tabelas A.3 e A.4) e qui-quadrado (Tabelas A.5 e A.6).

\section{Conclusões}

Como no conjunto anterior, observa-se um melhor desempenho dos estimadores para séries mais longas. Além disso, verificamos que quanto maior a razão de desvios (RD), maior será o módulo do viés dos estimadores. Observa-se também que quanto maior o valor de $\mathrm{RD}$, mais o valor de $d$ será subestimado. Este fato era esperado, pois quanto maior a variância do termo de memória curta, mais rápido será o decaimento para zero da função de autocorrelação. Exceto no caso de $d=0$, verifica-se que o estimador de Geweke e Porter-Hudak é o que apresenta o menor viés, porém maior variabilidade. O estimador do periodograma suavizado apresenta menor varibilidade, no entanto apresenta maior viés. Embora possua uma variância muito menor que os outros estimadores, o estimador truncado de Whittle é o que apresenta o maior viés, no entanto, sempre apresenta um menor erro quadrático médio. Os resultados são os mesmos para erros com distribuições gaussiana ou qui-quadrado. As Figuras 5.5, 5.6, 5.7 e 5.8 mostram o resultado do impacto de RD sob os estimadores para $T=1000$.

\subsubsection{Conjunto 3: Variações no Termo de Memória Longa}

O objetivo deste conjunto de simulações é avaliar o desempenho dos estimadores de $d$ em relação à variações dos coeficientes autoregressivos e de médias móveis no processo de memória longa. Em outras palavras, considere o processo

$$
y_{t}=x_{t}+\lambda w_{t}
$$

onde $\phi(B)(1-B)^{d} x_{t}=\theta(B) \epsilon_{t}$. Ou seja, $x_{t}$ é um processo $\operatorname{ARFIMA}(1, \mathrm{~d}, 1)$ e $w_{t}$ é um ruído branco. De forma a padronizar as simulações, consideramos $\operatorname{var}\left[x_{t}\right]=1, \operatorname{var}\left[w_{t}\right]=1$, $\lambda=1$ e $d=0,3$. Deseja-se verificar o comportamento dos estimadores $d_{G P H}, d_{S}$ e $d_{T W}$ 


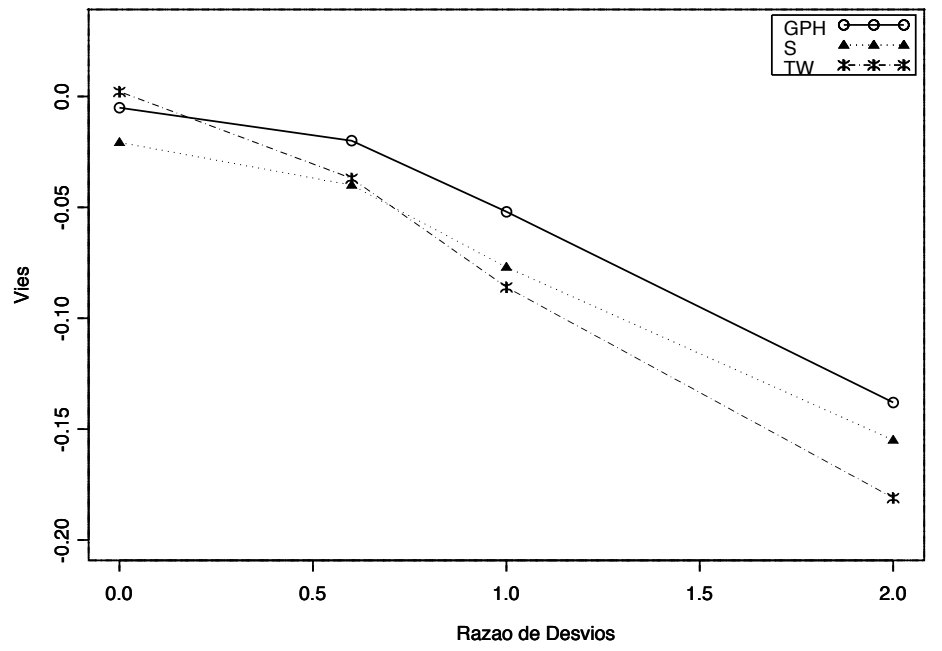

Figura 5.5: Viés dos Estimadores para variações da Razão de Desvios e Erros Gaussianos.

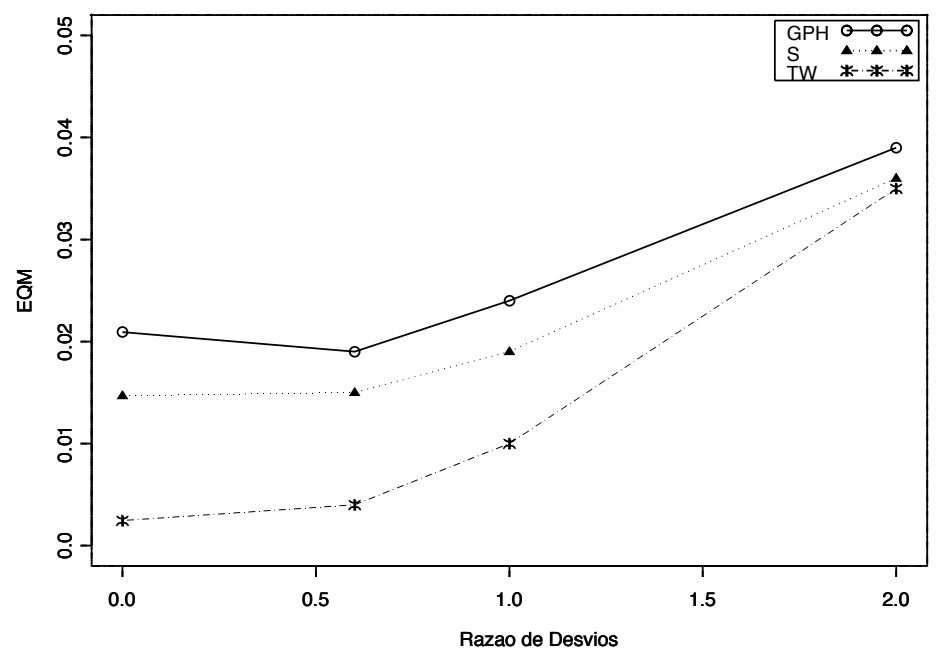

Figura 5.6: Erro Quadrático Médio dos Estimadores para variações da Razão de Desvios e Erros Gaussianos. 


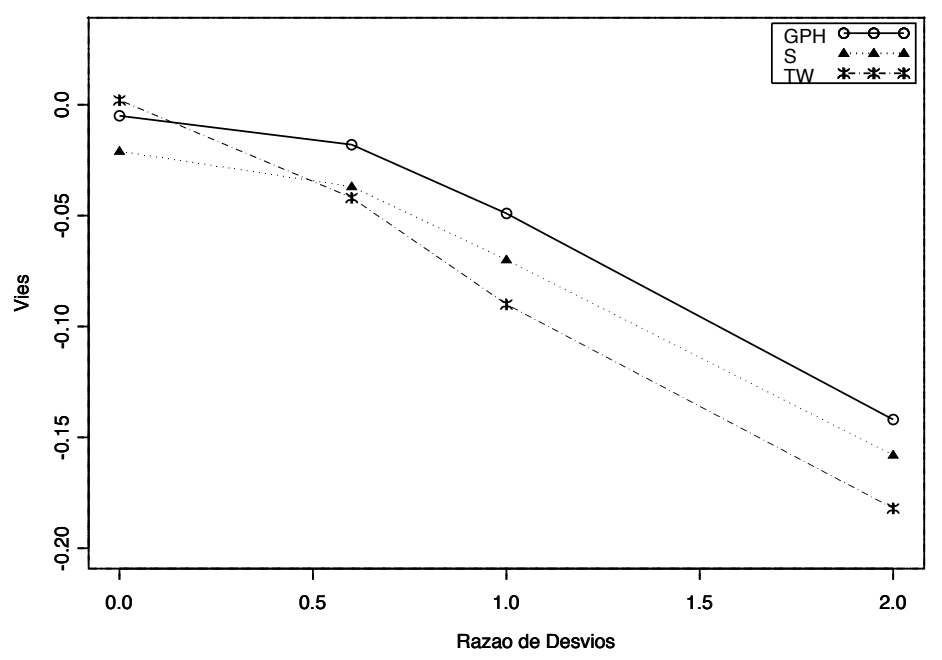

Figura 5.7: Viés dos Estimadores para variações da Razão de Desvios e Erros Quiquadrado.

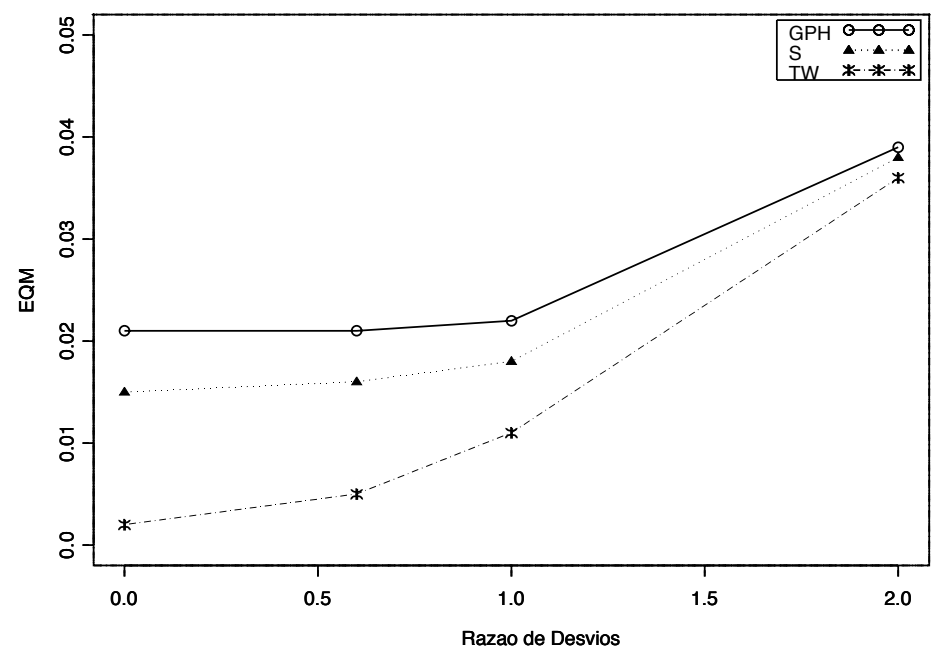

Figura 5.8: Erro Quadrático Médio dos Estimadores para variações da Razão de Desvios e Erros Qui-quadrado. 
em relação aos coeficientes $\theta$ e $\phi$. Os coeficientes $\theta$ e $\phi$ estão no intervalo [-0,99;0,99], mas seus efeitos foram analisados separadamente, ou seja, se $\theta \neq 0$ então $\phi=0$ e vice-versa. Os resultados das simulações estão nas Tabelas A.7 e A.8.

\section{Conclusões}

Primeiramente, observa-se que os estimadores possuem um melhor desempenho para um maior tamanho das séries. Além disso, verifica-se que valores negativos de $\phi$ e $\theta$ praticamente não afetam a estimação de $d$ (com exceção do caso de $\phi$ na fronteira de não-estacionariedade), porém apresentam viés positivo para valores positivos de $\phi$ e viés negativo para valores positivos de $\theta$.

Nota-se que embora com pouco viés, o estimador de Geweke e Porter-Hudak apresenta maior variabilidade. O estimador do periodograma suavizado apresenta um viés negativo, porém menor variância, de forma que apresenta menor erro quadrático médio. O estimador truncado de Whittle apresenta a menor variabilidade, porém verifica-se que para coeficientes positivos é o mais afetado. As Figuras 5.9, 5.10, 5.11 e 5.12 mostram os resultados da influência dos coeficientes $\phi$ e $\theta$ nos estimadores de $d$.

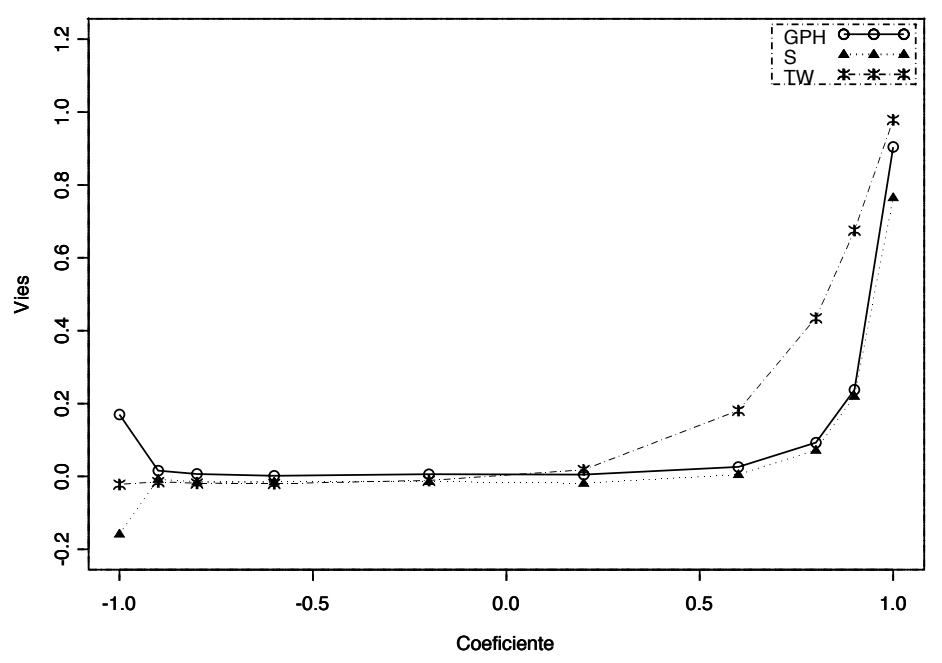

Figura 5.9: Viés dos Estimadores para variações em $\phi$ do processo de ARFIMA. 


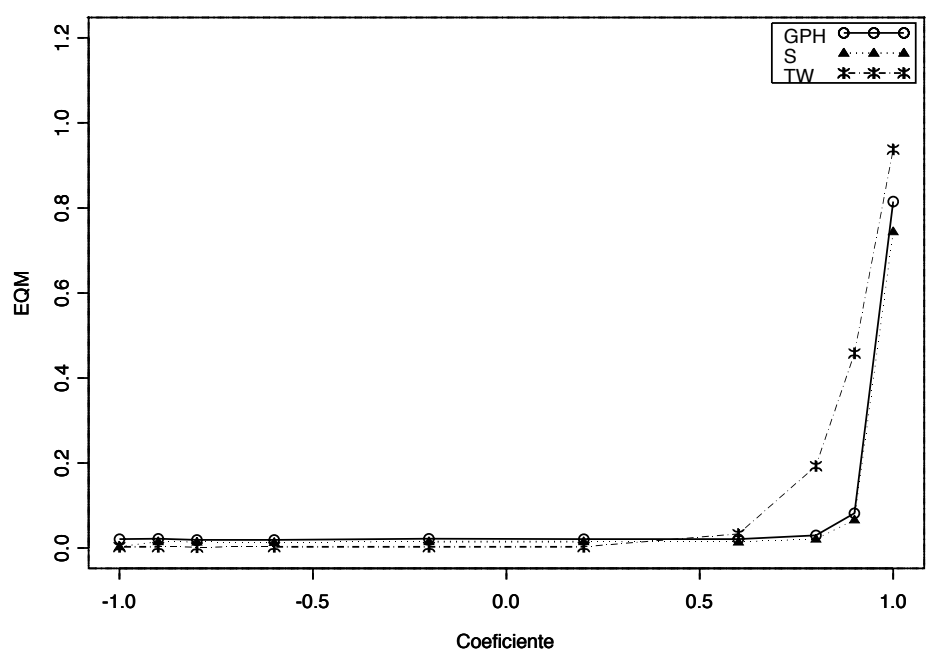

Figura 5.10: Erro Quadrático Médio dos Estimadores para variações em $\phi$ do processo de ARFIMA.

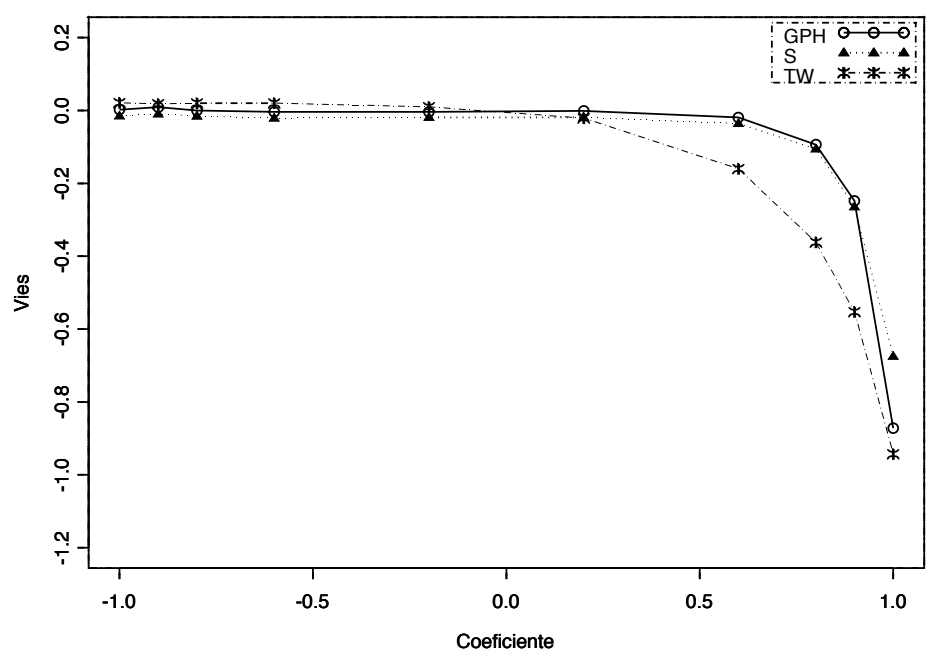

Figura 5.11: Viés dos Estimadores para variações em $\theta$ do processo de ARFIMA. 


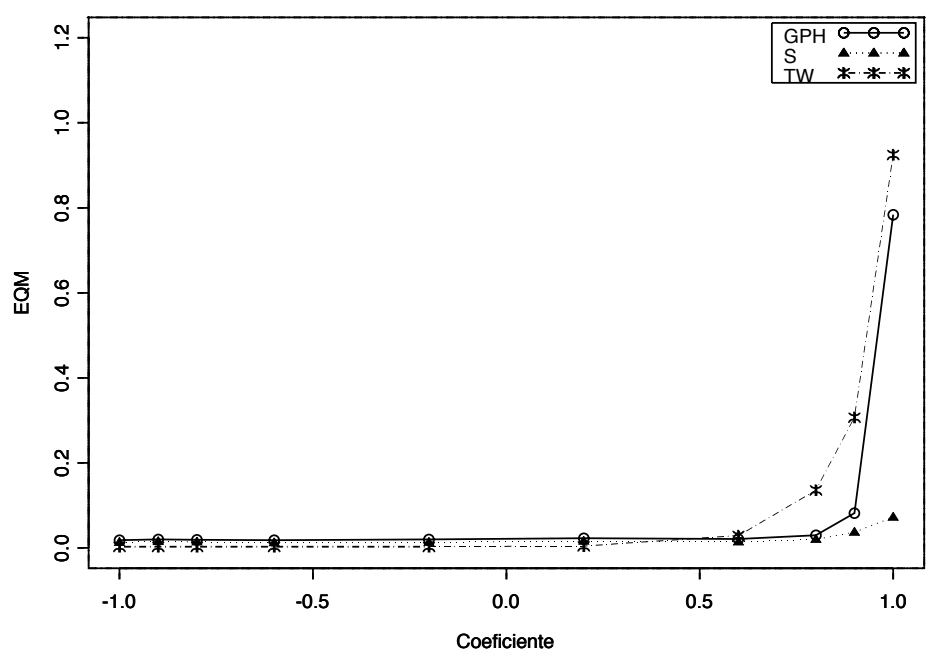

Figura 5.12: Erro Quadrático Médio dos Estimadores para variações em $\theta$ do processo de ARFIMA.

\subsubsection{Conjunto 4: Variações no Termo de Memória Curta}

Este conjunto de simulações foi realizado com objetivo semelhante ao conjunto anterior, no entanto, neste caso deseja-se avaliar o comportamento dos estimadores em relação à variações dos coeficientes autoregressivos e de médias móveis no processo de memória curta $w_{t}$. Considere o seguinte processo

$$
y_{t}=x_{t}+\lambda w_{t}
$$

onde $\phi(B) w_{t}=\theta(B) \epsilon_{t}$. Em outras palavras, o processo $w_{t}$ é um $\operatorname{ARMA}(1,1)$ e $x_{t}$ é um ruído branco fracionário. Padronizando as simulações, assumimos $\operatorname{var}\left[x_{t}\right]=1$, $\operatorname{var}\left[w_{t}\right]=1, \lambda=1$ e $d=0,3$ como no caso anterior. Os coeficientes $\theta$ e $\phi$ também estão no intervalo [-0,99;0,99], com efeitos analisados separadamente. Verificou-se também o desempenho dos estimadores assumindo $w_{t}$ com distribuições gaussiana (Tabelas A.9 e A.10) e qui-quadrado(Tabelas A.11 e A.12).

\section{Conclusões}

Novamente, verificamos que quanto mais longa for a série, melhor o desempenho dos estimadores. Como no caso anterior, observamos que os coeficientes $\phi$ e $\theta$ influênciam 
a estimação do parâmetro de memória longa. Porém, neste caso, verifica-se que valores negativos também possuem influência. Observa-se que o viés é negativo para $\phi<0,5$, mas para $\phi>0,5$ começa a tornar-se viesado positivamente. Além disso, observa-se que o viés é crescente em relação a $\theta$.

O estimador de Geweke e Porter-Hudak apresenta pouco viés, mas grande variabilidade. O estimador do periodograma suavizado apresenta um melhor desempenho, pois embora subestime o valor de $d$ possui menor variabilidade, e assim possui menor erro quadrático médio. Novamente, verifica-se que embora tenha variância pequena, o estimador truncado de Whittle é o mais afetado por variações em $\phi$ e $\theta$. Os resultados para erros com distribuições gaussiana e qui-quadrado são os mesmos. As Figuras 5.13, 5.14, 5.15, $5.16,5.17,5.18,5.19$ e 5.20 mostram os resultados para $T=1000$.

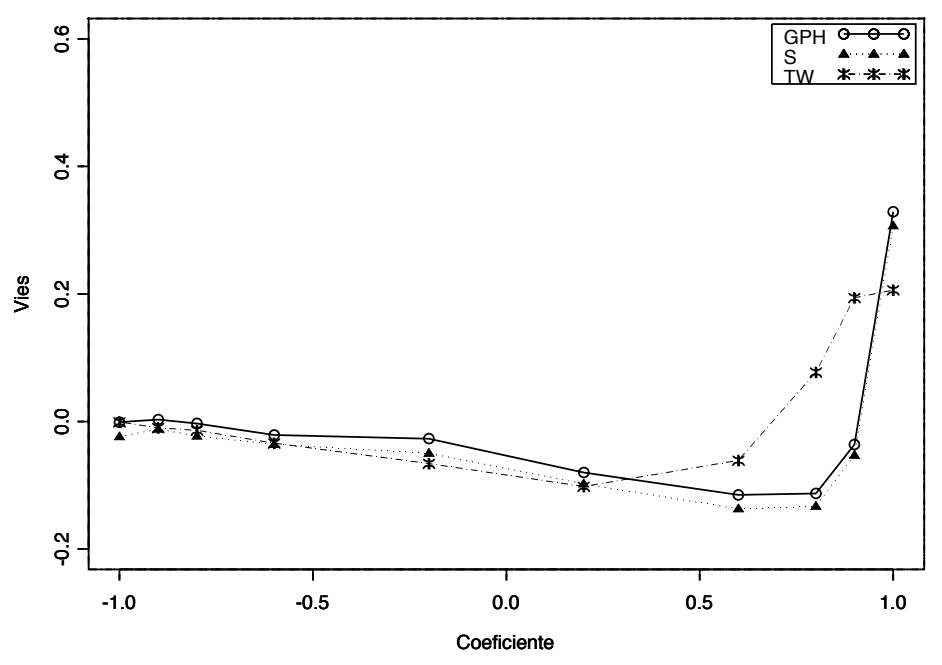

Figura 5.13: Viés dos Estimadores para variações em $\phi$ do processo de ARMA Gaussianos. 


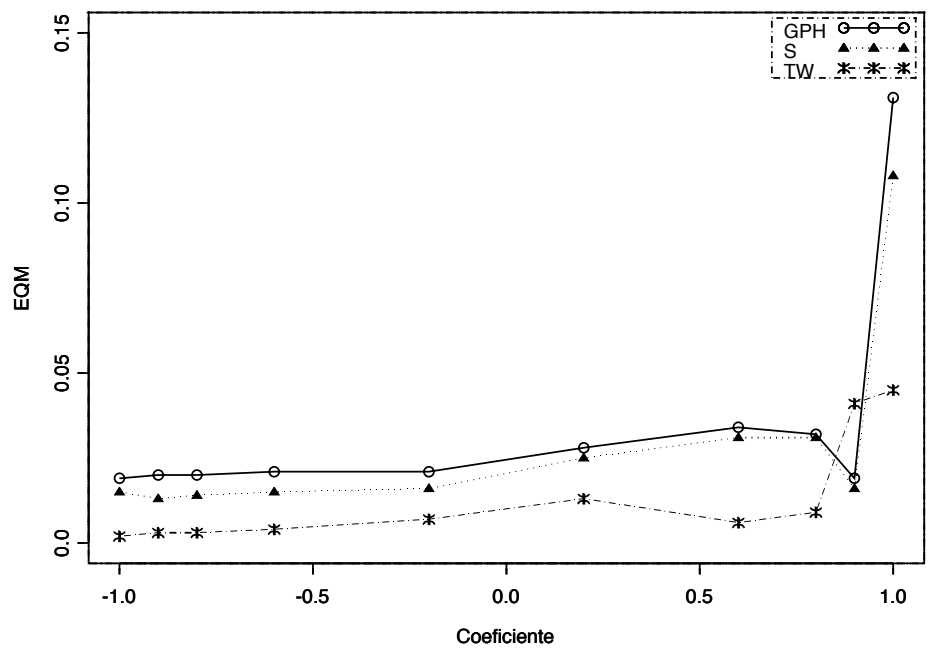

Figura 5.14: Erro Quadrático Médio dos Estimadores para variações em $\phi$ do processo de ARMA Gaussiano.

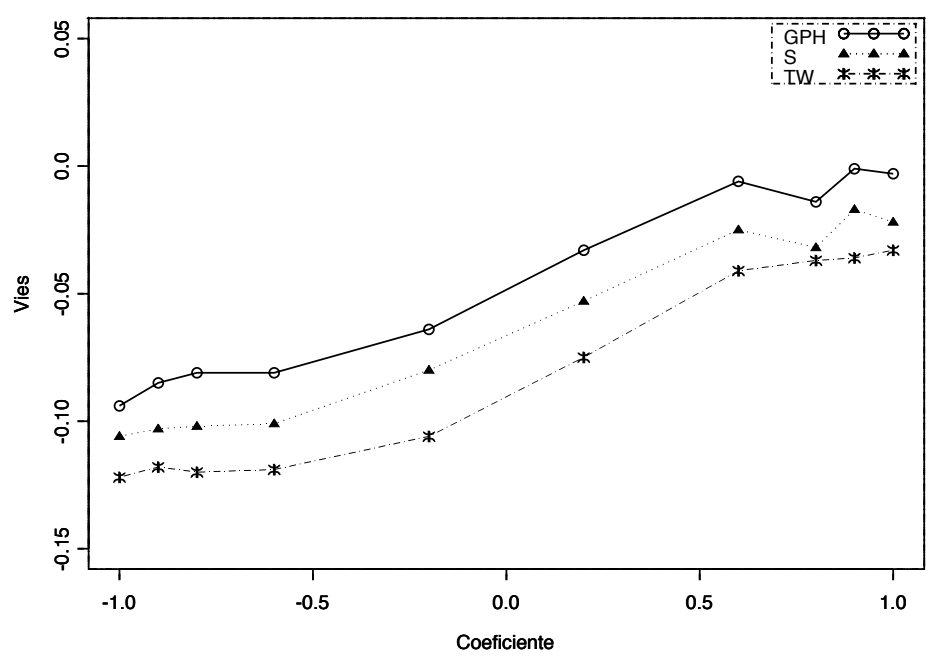

Figura 5.15: Viés dos Estimadores para variações em $\theta$ do processo de ARMA Gaussianos. 


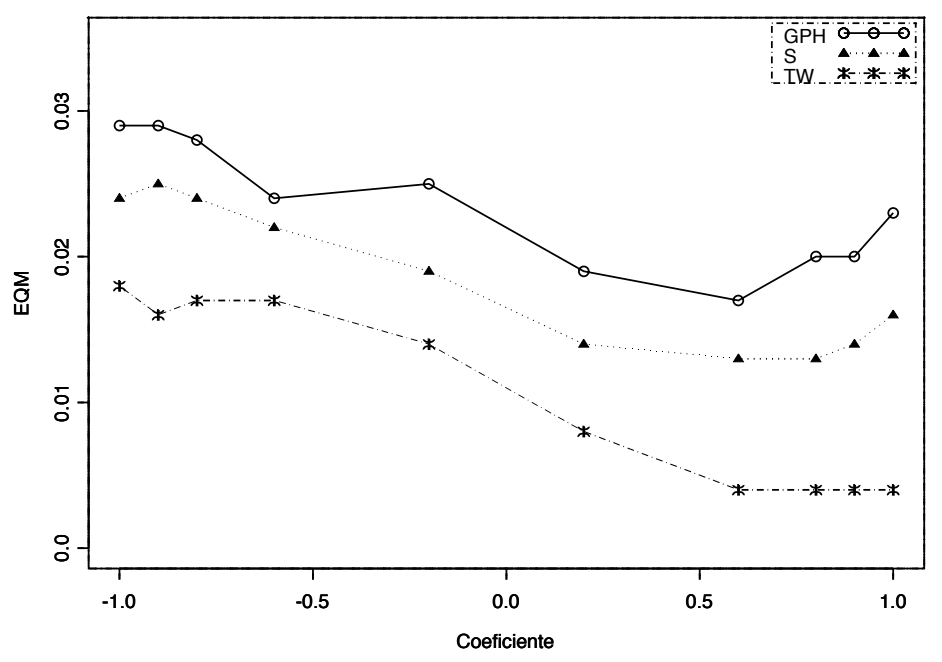

Figura 5.16: Erro Quadrático Médio dos Estimadores para variações em $\theta$ do processo de ARMA Gaussiano.

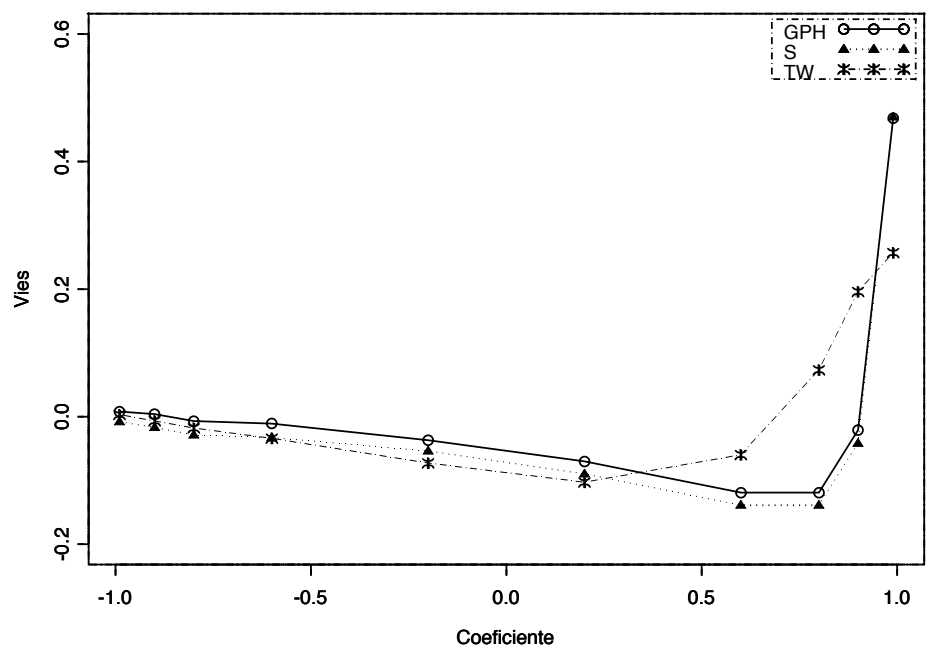

Figura 5.17: Viés dos Estimadores para variações em $\phi$ do processo de ARMA Quiquadrado. 


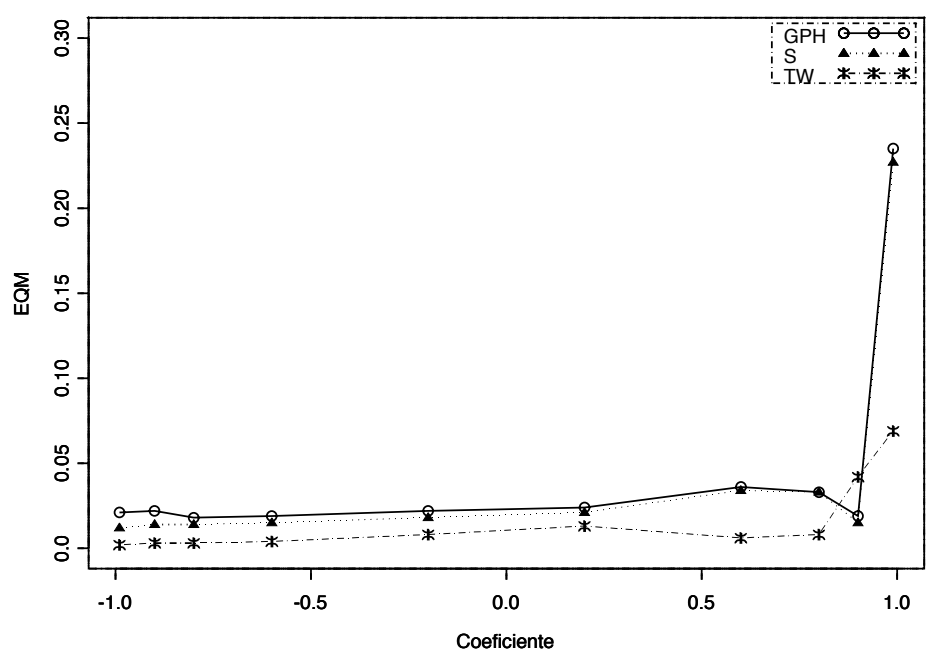

Figura 5.18: Erro Quadrático Médio dos Estimadores para variações em $\phi$ do processo de ARMA Qui-quadrado.

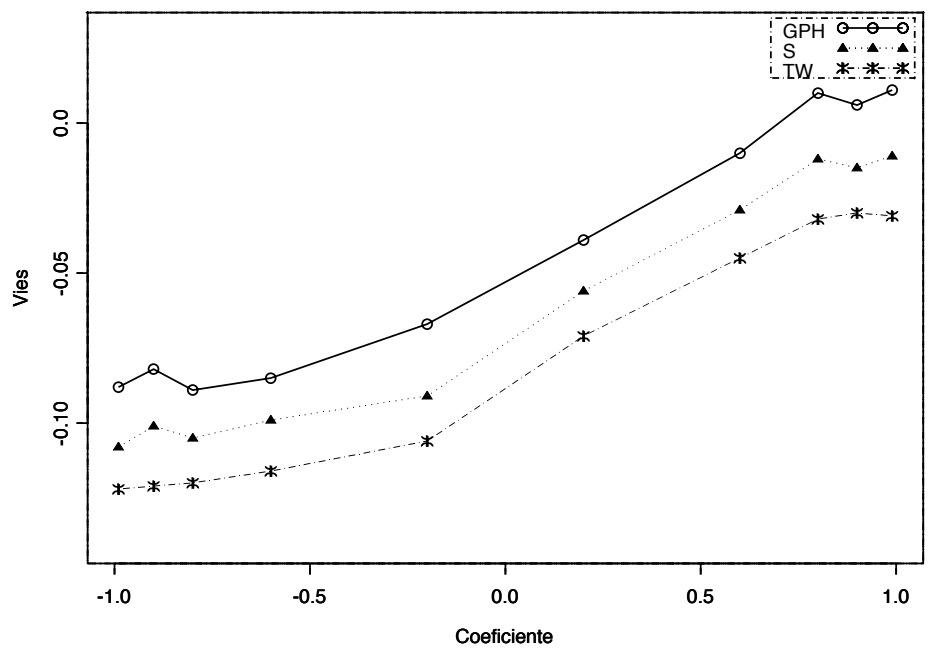

Figura 5.19: Viés dos Estimadores para variações em $\theta$ do processo de ARMA Quiquadrado. 


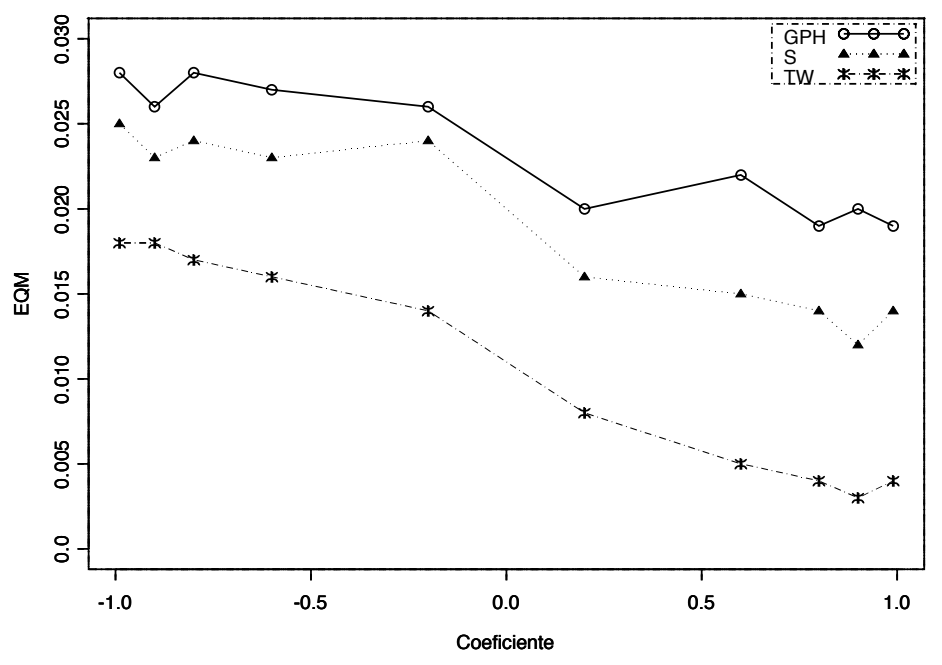

Figura 5.20: Erro Quadrático Médio dos Estimadores para variações em $\theta$ do processo de ARMA Qui-quadrado.

\subsection{Avaliação do Teste de Memória Longa Compar- tilhada}

Nesta seção, serão apresentados os resultados das simulações realizadas para avaliar o comportamento e o desempenho do teste de memória longa compartilhada através da análise de correlação canônica (Ray e Tsay, 1997).

\subsubsection{Conjunto 5: Teste MLC}

Considere o modelo de memória longa compartilhada bivariado (4.2)

$$
\begin{aligned}
& y_{1 t}=x_{t}+w_{1 t}, \\
& y_{2 t}=\lambda x_{t}+w_{2 t},
\end{aligned}
$$

onde $x_{t}$ é um ruído branco fracionário com parâmetro $d$ e $w_{i t}$ é um ruído branco, $i=1,2$. Neste conjunto de simulações, avaliamos o desempenho do teste de correlações canônicas para a estrutura de memória longa compartilhada (MLC). Em outras palavras, verificou- 
se o desempenho para o teste:

$$
\begin{aligned}
& H_{0}: y_{1 t} \text { e } y_{2 t} \text { possuem memória longa compartilhada; } \\
& H_{A}: y_{1 t} \text { e } y_{2 t} \text { não possuem memória longa compartilhada, }
\end{aligned}
$$

para diferentes valores dos parâmetros de defasagem $j$ e número de lags $h$, descritos no capítulo 4.

Foram realizadas $N=500$ simulações para tamanhos de séries $T=1000, d=0,4$, $\lambda=1, \operatorname{var}\left[x_{t}\right]=1$ e $\operatorname{var}\left[w_{i t}\right]=1$. Três tipos de simulações foram realizadas: para $j=3$ e $h=5$, para $j=5$ e $h=5$, e para $j=5$ e $h=10$. Além disso, também consideramos os casos assumindo $w_{i t}$ com distribuições gaussiana (Tabela B.1) e qui-quadrado (Tabela B.2). As tabelas mostram os quantis estimados das estatísticas $T$ e $T^{*}$ descritas no capítulo 4, o quantil esperado da distribuição qui-quadrado, a proporção estimada da rejeição da hipótese de MLC com nível de significância de 5\%, e a proporção de rejeição da hipótese de que a combinação linear $y^{*}$ possui memória curta com nível de significância de $5 \%$, utilizando o estimador truncado de Whittle.

\section{Conclusões}

Verifica-se que, independentemente da distribuição do termo de memória curta ou dos parâmetros $\mathrm{h}$ e j, os valores observados das estatísticas $T$ e $T^{*}$ estão muito próximas dos respectivos valores esperados. Além disso, verifica-se que a proporção de rejeição do teste de memória longa utilizando o estimador Truncado de Whittle, possui valores próximos ao nível de significância (5\%) indicando que o teste é adequado.

\subsubsection{Conjunto 6: Teste MLC - Variações no Termo de Memória Longa}

O objetivo deste conjunto de simulações é avaliar o impacto de variações nos coeficientes do termo de memória longa no teste $M L C$. Considere o modelo:

$$
\begin{aligned}
& y_{1 t}=x_{t}+w_{1 t}, \\
& y_{2 t}=\lambda x_{t}+w_{2 t},
\end{aligned}
$$

onde $\phi(B)(1-B)^{d}\left(x_{t}\right)=\theta(B) \epsilon_{t}$ é um processo $\operatorname{ARFIMA}(1, \mathrm{~d}, 1)$ e $w_{i t}$ é um ruído branco, $i=1,2$. Como no conjunto anterior, foram realizadas $N=500$ simulações, assumindo $T=1000, d=0,4, \lambda=1, \operatorname{var}\left[x_{t}\right]=1$ e $\operatorname{var}\left[w_{i t}\right]=1$. Três tipos de simulações foram realizadas: para $j=3$ e $h=5$, para $j=5$ e $h=5$, e para $j=5$ e $h=10$. Considerou-se 
também $w_{i t}$ com distribuição gaussiana. Utilizaram-se os valores - $-0,6,-0,2,0,2$ e 0,6 para os coeficientes $\phi$ (Tabela B.3) e $\theta$ (Tabela B.4), mas seus efeitos foram analisados separadamente, ou seja, se $\theta \neq 0$ então $\phi=0$ e vice-versa.

\section{Conclusões}

Verifica-se que os valores observados das estatísticas $T$ e $T^{*}$ são muito próximos aos valores esperados independentemente do valor de $\phi$ e para valores negativos de $\theta$, de forma que as proporções de rejeição da hipótese de MLC estão próximas ao nível de significância (5\%). No entanto, se testarmos se as combinações $y^{*}$ apresentam memória longa, concluimos que temos um melhor desempenho para valores de $h=5$. Porém, verifica-se que para valores grandes do coeficiente $\theta$, as estatísticas $T$ e $T^{*}$ subestimam o valor esperado, de forma que a proporção de rejeição é menor que nível de significância, independentemente do valor de j ou h. Verifica-se neste caso, que a proporção de rejeição da hipótese de que a combinação $y^{*}$ possua memória curta é maior que o nível de significância. Dessa forma, concluimos que o teste não é adequado para valores grandes do coeficiente $\theta$ do processo de memória longa.

\subsubsection{Conjunto 7: Teste MLC - Variações no Termo de Memória Curta}

Este último conjunto de simulações é semelhante ao anterior, mas tem como objetivo a avaliação do efeito de variações nos coeficientes do termo de memória curta no teste $M L C$. Considere o modelo:

$$
\begin{aligned}
& y_{1 t}=x_{t}+w_{1 t}, \\
& y_{2 t}=\lambda x_{t}+w_{2 t},
\end{aligned}
$$

onde $\phi(B)\left(w_{i t}\right)=\theta(B) \epsilon_{t}$ é um processo $\operatorname{ARMA}(1,1)$ e $x_{t}$ é um ruído branco fracionário, $i=1,2$. Simulou-se o modelo $N=500$ vezes, assumindo que o tamanho da série é $T=1000, d=0,4, \lambda=1, \operatorname{var}\left[x_{t}\right]=1$ e $\operatorname{var}\left[w_{i t}\right]=1$. Novamente três tipos de simulações foram realizadas: para $j=3$ e $h=5$, para $j=5$ e $h=5$, e para $j=5$ e $h=10$. Considerou-se também $w_{i t}$ com distribuição gaussiana e qui-quadrada. Utilizaram-se os valores $-0,6,-0,2,0,2$ e 0,6 para os coeficientes $\phi$ (Tabelas B.5 e B.7) e $\theta$ (Tabelas B.6 e B.8), mas seus efeitos foram analisados separadamente, ou seja, se $\theta \neq 0$ então $\phi=0$ e vice-versa. Lembremos que neste caso, a combinação linear $y^{*}$ é autocorrelacionada, 
e portanto o estimador truncado de Whittle não apresenta um bom desempenho, como visto no Conjunto 1. Dessa forma, para testarmos a hipótese de memória curta de $y^{*}$ utilizaremos o estimador do periodograma suavizado.

\section{Conclusões}

Através deste conjunto de simulações foi possível verificar diversas propriedades importantes das estatísticas para o teste MLC, principalmente devido ao fato de que na prática, as combinações lineares $y^{*}$ apresentam-se autocorrelacionadas.

Primeiramente verifica-se que no caso autoregressivo a estatística $T^{*}$ apresenta um desempenho indiscutivelmente melhor que a estatística $T$. Observa-se que a estatística $T$ superestima os valores dos quantis da $\chi^{2}$. Porém a estatística $T^{*}$ subestima os quantis para valores grandes de $h$. Além disso verifica-se que quanto maior o valor do módulo de $\phi$, menos eficaz é a estatística $T$.

Para o caso do termo de memória curta com médias móveis, verifica-se que a estatística $T$ apresenta um melhor desempenho do que a estatística $T^{*}$. Observa-se neste caso, que a estatística $T^{*}$ subestima os quantis da $\chi^{2}$. Além disso, nota-se que quanto maior o valor do módulo de $\theta$, menos eficaz é a estatística $T^{*}$.

Os resultados são os mesmo tanto para termos de memória curta com distribuição gaussiana quanto para erros com distribuição qui-quadrado. Em suma, verifica-se que para o caso autoregressivo a estatística $T^{*}$ é mais eficaz, e para o caso de médias móveis a estatística $T$ é a que apresenta o melhor desempenho.

\subsection{Síntese de Conclusões}

\subsubsection{Desempenho dos Estimadores de $d$}

Fazendo uma análise dos resultados dos conjuntos 1 ao 4, em síntese, temos:

- os três estimadores apresentam melhor desempenho quanto maior o número de observações das séries;

- variações nos coeficientes autoregressivos $(\phi)$ e de médias móveis $(\theta)$ tanto do 
termo de memória longa (ARFIMA) quanto do termo de memória curta (ARMA) produzem viés na estimativa de $d$ para os três estimadores;

- para processos ARMA "puros", valores grandes de $\phi$ produzem superestimativas de $d$. No entanto, valores grandes de $\theta$ produzem viés negativo;

- para processos ARFIMA + ARMA, valores grandes de $\phi$ do termo ARFIMA produzem superestimativas de $d$, por outro lado, valores grandes de $\theta$ produzem viés negativo;

- para processos ARFIMA + ARMA, o viés é negativo para $\phi<0,5$ e torna-se crescente positivo para $\phi>0,5$ do processo de ARMA. Além disso, nota-se que o módulo do viés é crescente para $\theta$, no entanto o viés é sempre negativo;

- o estimador de Geweke e Porter-Hudak $\left(\hat{d}_{G P H}\right)$ embora com pouco viés, apresenta maior variabilidade;

- o estimador do periodograma suavizado $\left(\hat{d}_{S}\right)$ utilizando a janela de BartlettPriestley, embora apresente um ligeiro viés negativo, possui menor variância que $\left(\hat{d}_{G P H}\right)$, de forma que apresenta um menor erro quadrático médio;

- o estimador truncado de Whittle $\left(\hat{d}_{T W}\right)$ é o que apresenta a menor variabilidade. Porém verifica-se que é o mais afetado por variações de coeficientes $\phi$ e $\theta$ tanto do termo ARMA quanto do termo ARFIMA. Ou seja, é de grande eficiência para testar a hipótese $H_{0}: d=0$ vs $H_{A}: d \neq 0$ na ausência de termos autogressivos e de médias móveis, porém o teste apresenta um elevado erro do tipo I, se estes coeficientes forem diferentes de zero;

- os resultados são os mesmos para as distribuições do termo de memória curta gaussiana ou qui-quadrado.

\subsubsection{Desempenho do Teste MLC}

Analisando o desempenho do teste de memória longa compartilhada através da análise de correlação canônica nos conjuntos de 5 a 7 , os principais resultados são:

- no caso Ruído Branco Fracionário + Ruido Branco, os quantis das estatísticas T e $T^{*}$ assumem valores bem próximos aos esperados, e a combinação linear $y^{*}$ apresenta $d=0$ indicando que metodologia é adequada;

- para o caso em que o termo de memória longa é um processo ARFIMA, o teste é adequado para variações dos coeficientes $\phi$ e $\theta<0$, porém para valores grandes de $\theta$ os quantis das estatísticas $T$ e $T^{*}$ subestimam o valor verdadeiro da $\chi^{2}$; 
- se o termo de memória curta é um processo ARMA, as estatísticas $T$ e $T^{*}$ possuem características peculiares. No caso autoregressivo verifica-se que a estatística $T^{*}$ apresenta um melhor desempenho, pois a $T$ superestima os quantis esperados. No caso de médias móveis, verifica-se que a estatística $T$ apresenta o melhor desempenho, pois $T^{*}$ subestima os quantis esperados;

- é interessante observar que embora os coeficientes das combinações lineares $y^{*}$ sejam estimados, nas simulações realizadas observa-se que $y^{*}$ apresenta memória curta, indicando que a metodologia é adequada;

- os resultados são os mesmo tanto para processos com termos de memória curta com distribuição gaussiana ou qui-quadrado. 


\section{CAPÍTULO 6}

\section{Aplicações}

Neste capítulo serão apresentadas algumas aplicações a dados reais dos estimadores do parâmetro de memória longa e do teste de memória longa compartilhada através da análise de correlação canônica. A primeira aplicação foi realizada a dados diários da velocidade do vento de São Paulo e Piracicaba. Em seguida, serão descritas duas aplicações na área financeira, em séries de volatilidade de índices de bolsas de valores.

\subsection{Velocidade do Vento}

Os dados desta aplicação são provenientes de estações meteorológicas situadas nos municípios de São Paulo e Piracicaba. O posto meteorológico do município de São Paulo, situa-se junto às dependências Faculdade de Filosofia, Letras e Ciências Humanas da Universidade de São Paulo (FFLCH). No município de Piracicaba, temos um posto agrometeorológico na Escola Superior de Agricultura Luiz de Queiroz (ESALQ), também pertencente à Universidade de São Paulo. Os dados estão disponíveis na Internet.

A velocidade do vento $(\mathrm{m} / \mathrm{s})$ foi medida a 2 metros de altura, entre 01/01/2000 e 26/05/2001, de forma que as séries possuem 512 observações. Para estabilizar a variância das séries, para todas as análise realizadas, considerou-se o logaritmo das mesmas. $\mathrm{Na}$ Figura 6.1 encontram-se o logaritmo das séries e os respectivos periodogramas. A Figura 6.2 descreve as funções de autocorrelação e autocorrelação cruzada.

Note que as funções de autocorrelação possuem lento decaimento para zero, indicando que as séries possuem memória longa. Além disso, verifica-se que as funções de auto- 

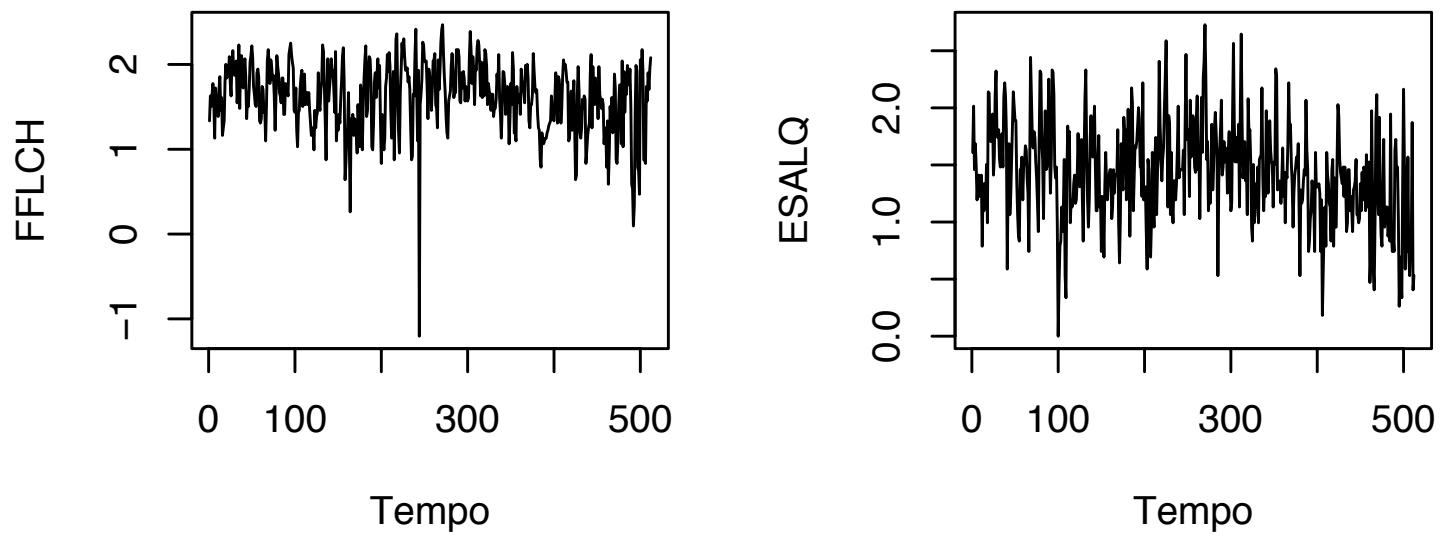

FFLCH
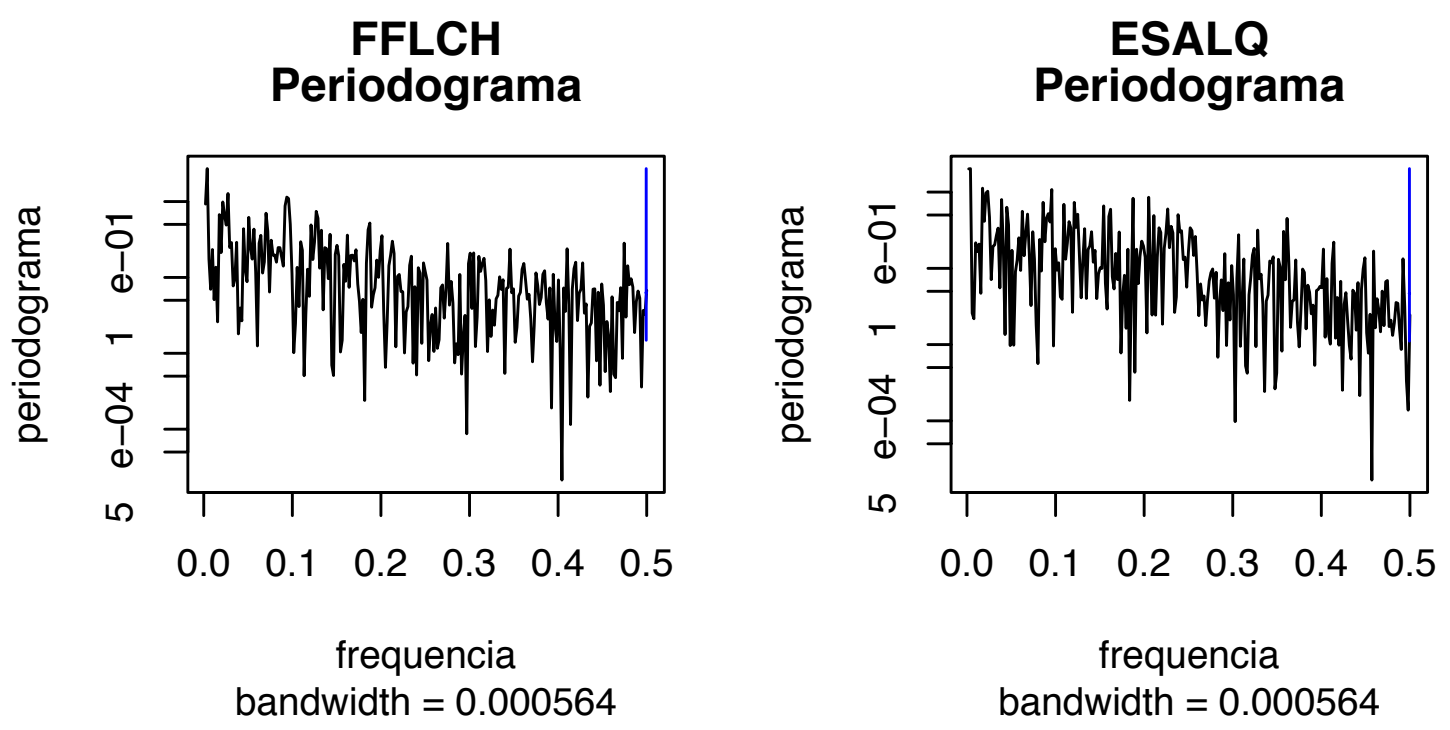

Figura 6.1: Logaritmo das séries de velocidade do vento e os respectivos periodogramas. 
FFLCH

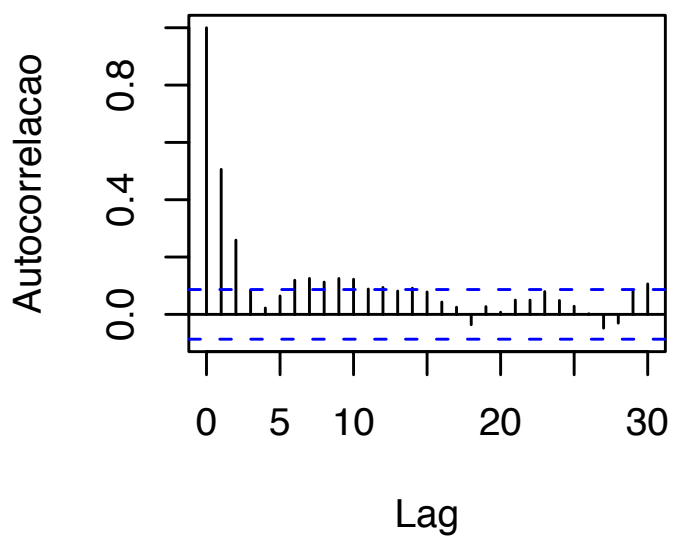

ESALQ \& FFLCH

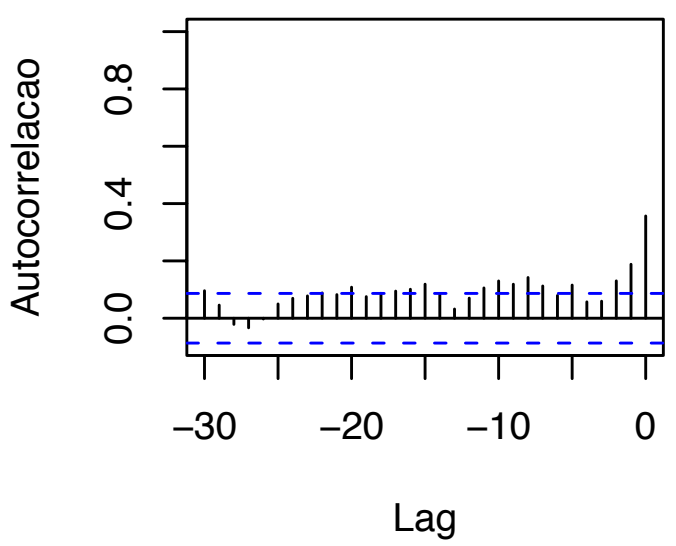

FFLCH \& ESALQ

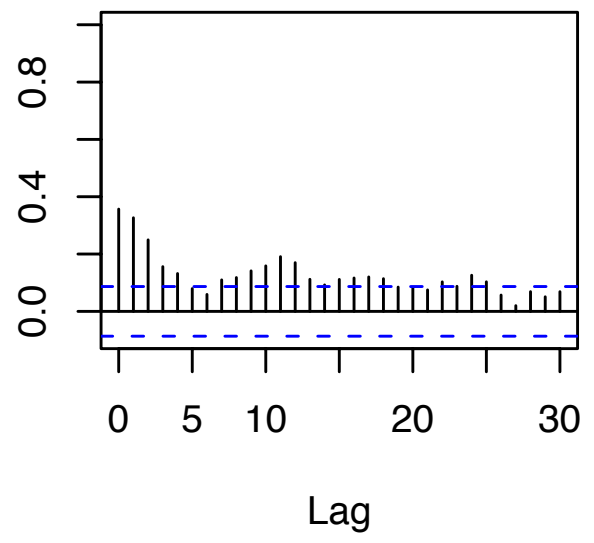

ESALQ

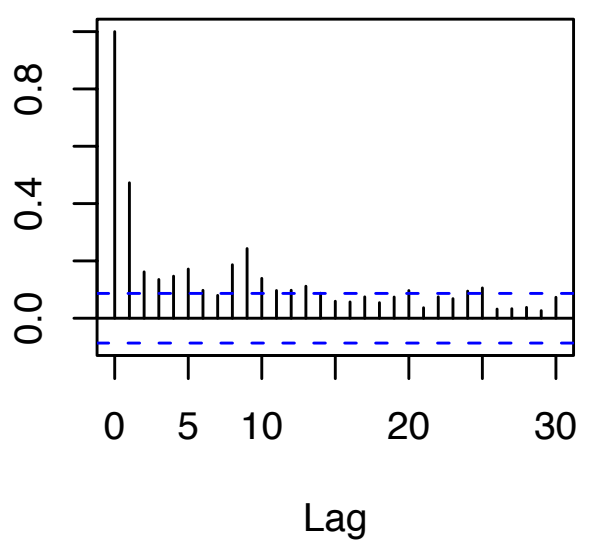

Figura 6.2: Funções de autocorrelação e autocorrelação cruzada das séries. 
correlação cruzada (FFLCH\&ESALQ) também decaem lentamente para zero, condição necessária para séries com memória longa compartilhada. Utilizando os estimadores descritos no capítulo 3, obtemos as estimativas descritas nas Tabelas 6.1 e 6.2.

Os estimadores também indicam que há presença de memória longa nas séries. Mesmo

\begin{tabular}{|c|ccc|}
\hline FFLCH & d & desv.pad. & p-valor \\
\hline$\hat{d}_{T W}$ & 0,187 & 0,0563 & 0,001 \\
$\hat{d}_{S}$ & 0,324 & 0,125 & 0,017 \\
$\hat{d}_{G P H}$ & 0,334 & 0,179 & 0,076 \\
\hline
\end{tabular}

Tabela 6.1: Estimação de $d$ da série de velocidade do vento da FFLCH.

\begin{tabular}{|c|ccc|}
\hline ESALQ & d & desv.pad. & p-valor \\
\hline$\hat{d}_{T W}$ & 0,225 & 0,056 & 0,000 \\
$\hat{d}_{S}$ & 0,288 & 0,125 & 0,032 \\
$\hat{d}_{G P H}$ & 0,310 & 0,166 & 0,077 \\
\hline
\end{tabular}

Tabela 6.2: Estimação de $d$ da série de velocidade do vento da ESALQ.

o estimador de Geweke e Porter-Hudak, que é o de menor poder, sugere que as séries possuem $d \neq 0$, a um nível significância de 0,08 . Na Tabela 6.3, estão os resultados do teste MLC para as duas séries $(j=2$ e $h=5)$, onde apresentamos as estatísticas $T$ e $T^{*}$ e os respectivos níveis descritivos para as correlações canônicas $\rho_{1}^{2}$ e $\rho_{2}^{2}$. Note que $T$ e $T^{*}$ correspondentes à $\rho_{1}^{2}$ são não significativas a $5 \%$, indicando que as séries possuem memória longa compartilhada.

Dessa forma, o teste MLC indica que as duas séries possuem memória longa com-

\begin{tabular}{|c|cc|cc|}
\hline & $T$ & p-valor & $T^{*}$ & p-valor \\
\hline$\rho_{1}^{2}$ & 10,972 & 0,278 & 8,567 & 0,478 \\
$\rho_{2}^{2}$ & 68,515 & 0,000 & 46,467 & 0,001 \\
\hline
\end{tabular}

Tabela 6.3: Estatísticas $T$ e $T^{*}$ para o teste de memória longa compartilhada da velocidade do vento na FFLCH e ESALQ.

partilhada. Portanto, deve existir uma combinação linear das séries que possua apenas memória curta. O vetor dessas combinações é dado por $(-0,525 ; 0,908)$, onde a primeira 
coordenada refere-se à série FFLCH. Para verificarmos se a combinação linear $\left(y^{*}\right)$ das séries possui memória longa, através da função de autocorrelação (Figura 6.3) e utilizando os estimadores de $d$ (Tabela 6.4).

Note que somente o teste utilizando o estimador Truncado de Whittle rejeita a
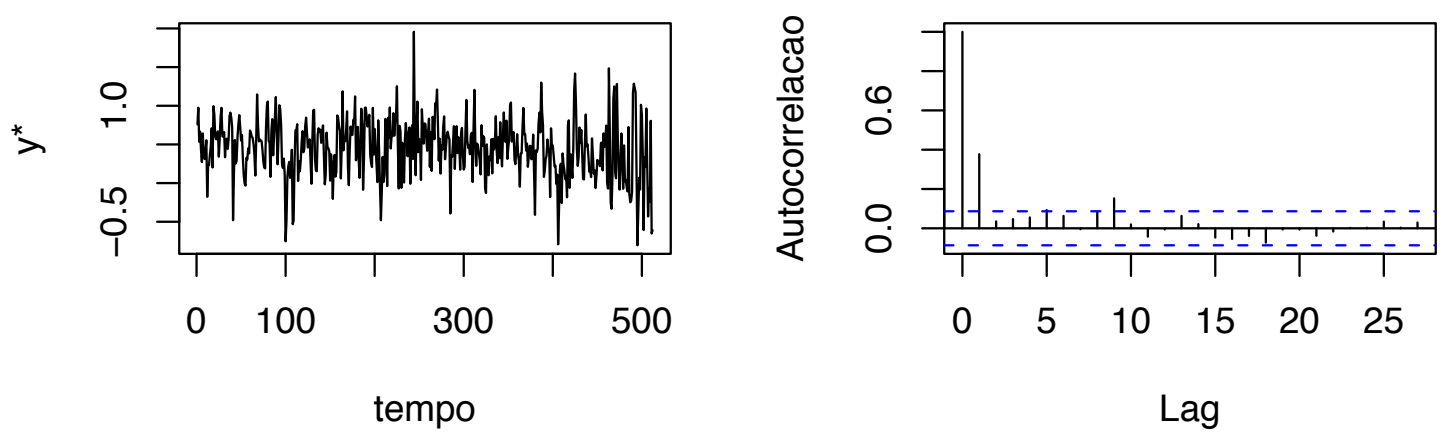

Figura 6.3: Combinação linear $y^{*}$.

\begin{tabular}{|c|ccc|}
\hline $\mathbf{y}$ & $\mathbf{d}$ & desvpad & p-valor \\
\hline$\hat{d}_{T W}$ & 0,164 & 0,056 & 0,005 \\
$\hat{d}_{S}$ & 0,022 & 0,125 & 0,862 \\
$\hat{d}_{G P H}$ & 0,097 & 0,203 & 0,637 \\
\hline
\end{tabular}

Tabela 6.4: Estimação de $d$ da combinação linear y.

hipótese de que $y$ possui $d=0$. Porém, observa-se que $y$ é autocorrelacionada, e segundo os resultados do conjunto de simulações 1 , neste caso o estimador de Whittle é viesado, e possui alto erro do tipo I. Portanto, não há evidências para rejeitar a hipótese que as séries do logaritmo da velocidade do vento nas duas estações meteorológicas possuam memória longa compartilhada. 


\subsection{Bolsas de Hong Kong, Nova Zelândia e Singapura}

Os dados desta aplicação são índices das bolsas de valores de Hong Kong (Hang Seng), Nova Zelândia (NZSE 40) e Singapura (Straits Times), e estão disponíveis no website da Reuters Limited.

Foram coletados índices entre 21/08/1997 a 12/08/2003, totalizando séries de tamanho 1549. Em nossa análise estamos interessados em verificar se a volatilidade das três bolsas de valores seguem um modelo de memória longa compartilhada. Dessa forma, as séries a serem utilizadas serão os logaritmos dos quadrados dos logretornos das séries $\left(\log \left(r_{t}^{2}\right)\right)$, pois a abordagem é coerente como o modelo de volatilidade estocástica de memória longa descrito no capítulo 3. É interessante lembrar que neste caso, os termos de memória curta possuem distribuição qui-quadrado, mas através das simulações descritas no capítulo 5, verificamos que isso não afeta os estimadores de $d$ e nem o teste MLC.

É importante alertarmos para o fato de que os retornos muitas vezes são próximos de zero, e o logaritmo não está muito bem definido para esse domínio. Dessa forma, utilizamos a transformação de Füller (1996) dada por

$$
x_{t}^{*}=\log \left(r_{t}^{2}+\lambda S_{r}^{2}\right)-\frac{\lambda S_{r}^{2}}{r_{t}^{2}+\lambda S_{r}^{2}},
$$

onde $r_{t}$ é o logretorno dos índices, $S_{r}^{2}$ a variância amostral dos logretornos e $\lambda=0,02$.

Os índices das bolsas estão descritos na Figura 6.4 e os respectivos periodogramas das séries de volatilidade (logaritmo do quadrado dos retornos) estão na Figura 6.5. As funções de autocorrelação podem ser encontradas na Figura 6.6.

Observa-se que as funções de autocorrelação decaem lentamente para zero, indicando que as séries de volatilidade apresentam persistência. As estimativas do parâmetro $d$ estão nas Tabelas 6.4, 6.5, 6.6 e 6.7.

Os testes também indicam que há evidências de que as séries de volatilidade possuem

\begin{tabular}{|c|ccc|}
\hline HK & d & desv.pad. & p-valor \\
\hline$\hat{d}_{T W}$ & 0,194 & 0,038 & 0,000 \\
$\hat{d}_{S}$ & 0,295 & 0,082 & 0,001 \\
$\hat{d}_{G P H}$ & 0,418 & 0,119 & 0,001 \\
\hline
\end{tabular}

Tabela 6.5: Estimação de $d$ da série de volatilidade da bolsa de Hong Kong. 

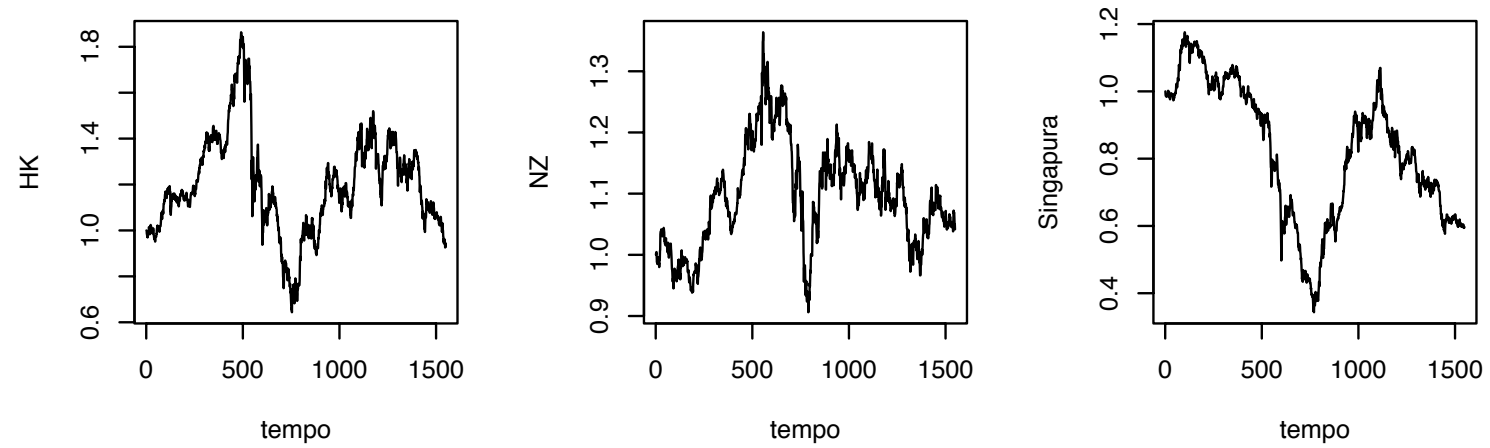

Figura 6.4: Índices das Bolsas de Valores.
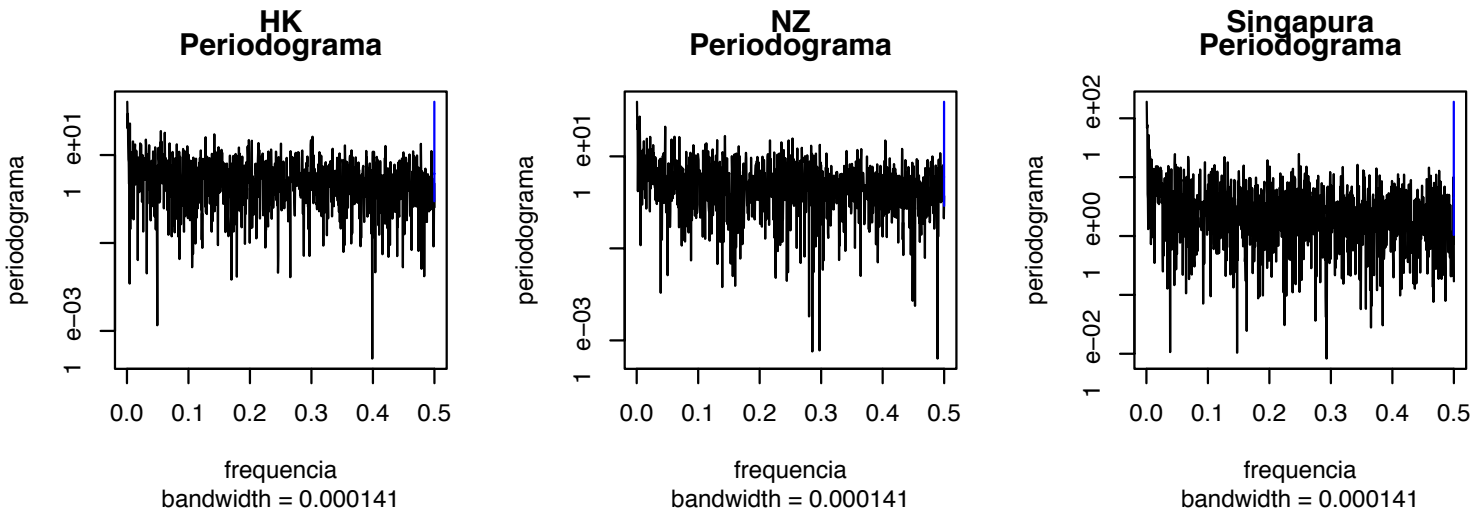

Figura 6.5: Periodograma das Séries de Volatilidade.

\begin{tabular}{|c|ccc|}
\hline $\mathbf{N Z}$ & $\mathbf{d}$ & desv.pad. & p-valor \\
\hline$\hat{d}_{T W}$ & 0,243 & 0,038 & 0,000 \\
$\hat{d}_{S}$ & 0,278 & 0,082 & 0,002 \\
$\hat{d}_{G P H}$ & 0,335 & 0,115 & 0,006 \\
\hline
\end{tabular}

Tabela 6.6: Estimação de $d$ da série de volatilidade da bolsa da Nova Zelândia. 

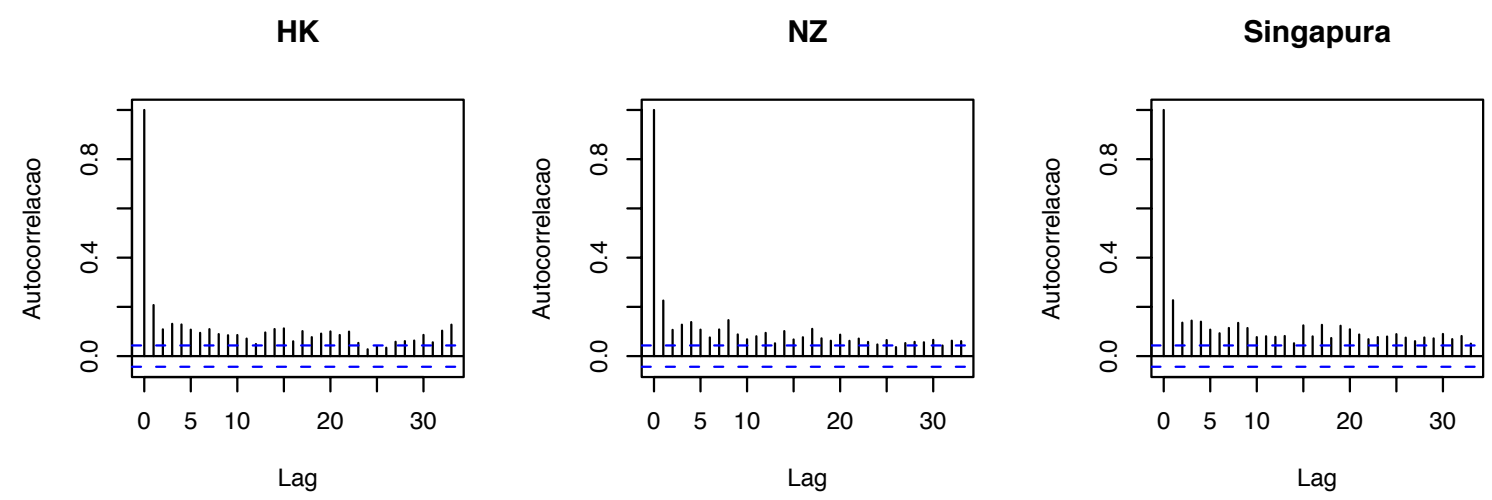

Figura 6.6: Funções de Autocorrelação das séries de volatilidade.

\begin{tabular}{|c|ccc|}
\hline Singapura & d & desv.pad. & p-valor \\
\hline$\hat{d}_{T W}$ & 0,205 & 0,038 & 0,000 \\
$\hat{d}_{S}$ & 0,235 & 0,082 & 0,007 \\
$\hat{d}_{G P H}$ & 0,273 & 0,166 & 0,108 \\
\hline
\end{tabular}

Tabela 6.7: Estimação de $d$ da série de volatilidade da bolsa de Singapura.

memória longa. O estimador de Geweke e Porter-Hudak não rejeita a hipótese de que a volatilidade da bolsa de Singapura é $I(0)$ a $5 \%$ de significância, no entanto, lembremos que é o estimador com maior variabilidade. Os resultados do teste MLC às séries estão na Tabela 6.8. Note que as estatísticas $T$ e $T^{*}$ referentes a $\rho_{1}^{2}$ e $\rho_{2}^{2}$ são não-significantes, indicando que as três séries possuem o mesmo componente de memória longa.

Assim, o teste MLC ( $j=3, h=5)$ indica que as séries de volatilidade das três bolsas

\begin{tabular}{|c|cc|cc|}
\hline & $T$ & p-valor & $T^{*}$ & p-valor \\
\hline$\rho_{1}^{2}$ & 7,801 & 0,648 & 8,046 & 0,624 \\
$\rho_{2}^{2}$ & 17,727 & 0,722 & 18,748 & 0,661 \\
$\rho_{3}^{2}$ & 140,182 & 0,000 & 86,962167 & 0,000 \\
\hline
\end{tabular}

Tabela 6.8: Estatísticas $T$ e $T^{*}$ para o teste de memória longa compartilhada da volatilidade das bolsas de Hong Kong, Nova Zelândia e Singapura.

possuem memória compartilhada. Além disso, como as estatísticas são não significativas para $\rho_{1}$ e $\rho_{2}$, isso significa que há um único processo de memória longa para as três séries. 
Portanto, é possível encontrar 2 combinações lineares das séries que possuem memória curta. Os vetores de coeficientes são $(-0,600 ;-0,077 ; 0,915)$ e $(-0,481 ; 0,672 ;-0,219)$, onde as coordenadas referem-se respectivamente às volatilidade das bolsas de Hong Kong, Nova Zelândia e Singapura. A Figura 6.7 descrevem as séries combinações lineares $\left(y_{1}^{*}\right.$ e $\left.y_{2}^{*}\right)$ e suas respectivas funções de autocorrelação.

Observando as funções de autocorrelação de $y_{1}^{*}$ e $y_{2}^{*}$, aparentemente as séries não possuem memória longa. As estimativas do parâmetro $d$ de $y_{1}^{*}$ e $y_{2}^{*}$ pode ser encontrada nas Tabelas 6.9 e 6.10 ,

Os testes também indicam que as séries $y_{1}$ e $y_{2}$ não possuem memória longa. Portanto,

\begin{tabular}{|c|ccc|}
\hline$y_{1}$ & d & desv.pad. & p-valor \\
\hline$\hat{d}_{T W}$ & 0,041 & 0,038 & 0,283 \\
$\hat{d}_{S}$ & 0,091 & 0,082 & 0,275 \\
$\hat{d}_{G P H}$ & 0,064 & 0,098 & 0,515 \\
\hline
\end{tabular}

Tabela 6.9: Estimação de $d$ da série combinação linear $y_{1}^{*}$.

\begin{tabular}{|c|ccc|}
\hline$y_{2}$ & d & desv.pad. & p-valor \\
\hline$\hat{d}_{T W}$ & 0,041 & 0,038 & 0,286 \\
$\hat{d}_{S}$ & 0,083 & 0,082 & 0,320 \\
$\hat{d}_{G P H}$ & $-0,011$ & 0,108 & 0,921 \\
\hline
\end{tabular}

Tabela 6.10: Estimação de $d$ da série combinação linear $y_{2}^{*}$.

não há evidências para rejeitar a hipótese de que as séries de volatilidades das bolsas de valores de Hong Kong, Nova Zelândia e Singapura possuam memória longa compartilhada, e o termo de memória longa é único.

\subsection{Bolsas de Valores do Brasil e Reino Unido}

Esta aplicação é similar à aplicação anterior, e os índices são do mesmo período. No entanto, deseja-se verificar se a volatilidade das bolsas do Brasil (BOVESPA) e do Reino Unido (FTSE100) possuem memória longa compartilhada. A Figura 6.8 descreve 

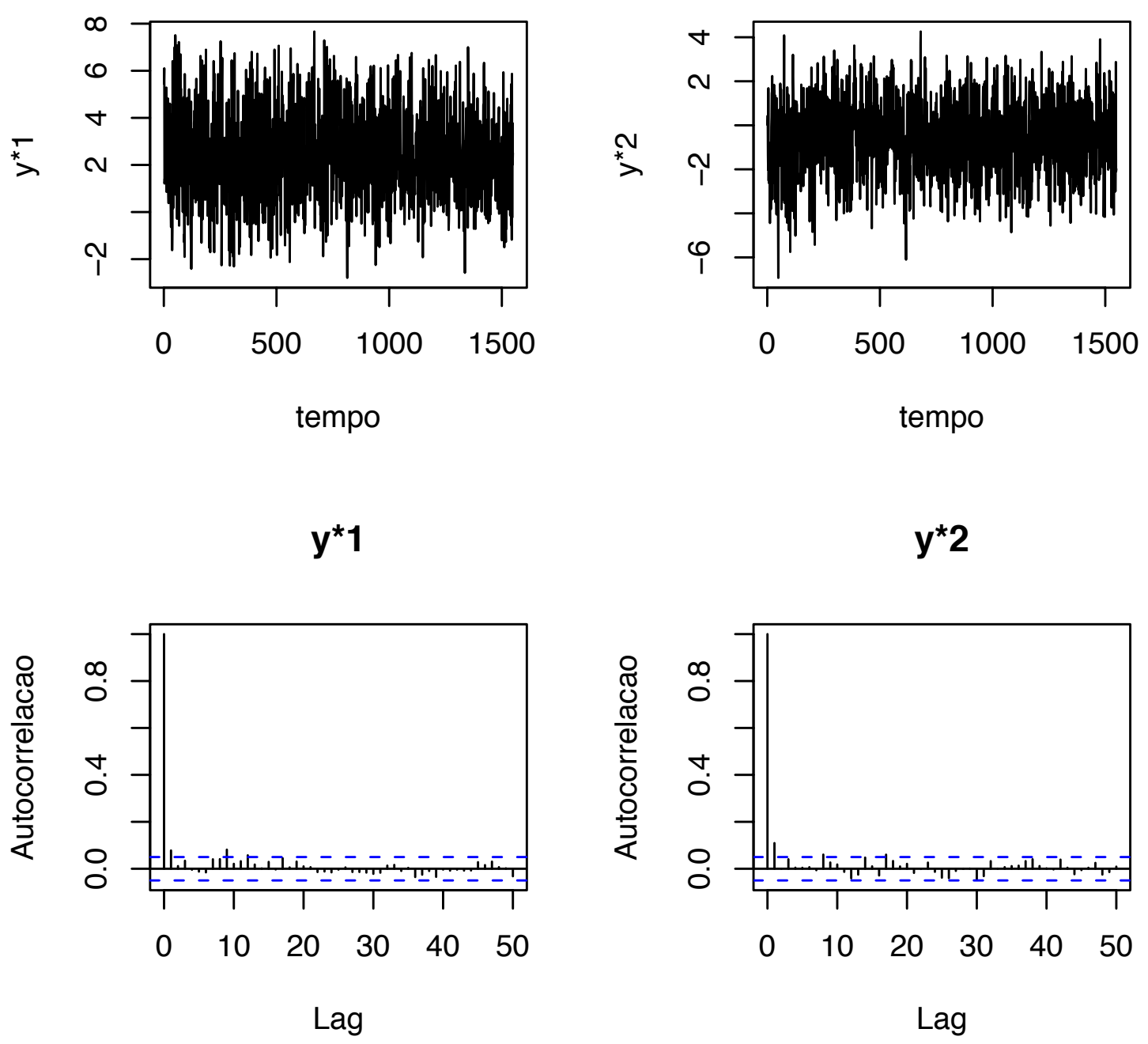

Figura 6.7: Funções de Autocorrelação das Combinações Lineares das Séries de volatilidade. 
os índices e periodograma da volatilidade da bolsa dos dois países. As funções de autocorrelação e autocorrelação cruzada das séries de volatilidade estão na Figura 6.9.

As funções de autocorrelação indicam que as séries possuem memória longa. Porém,
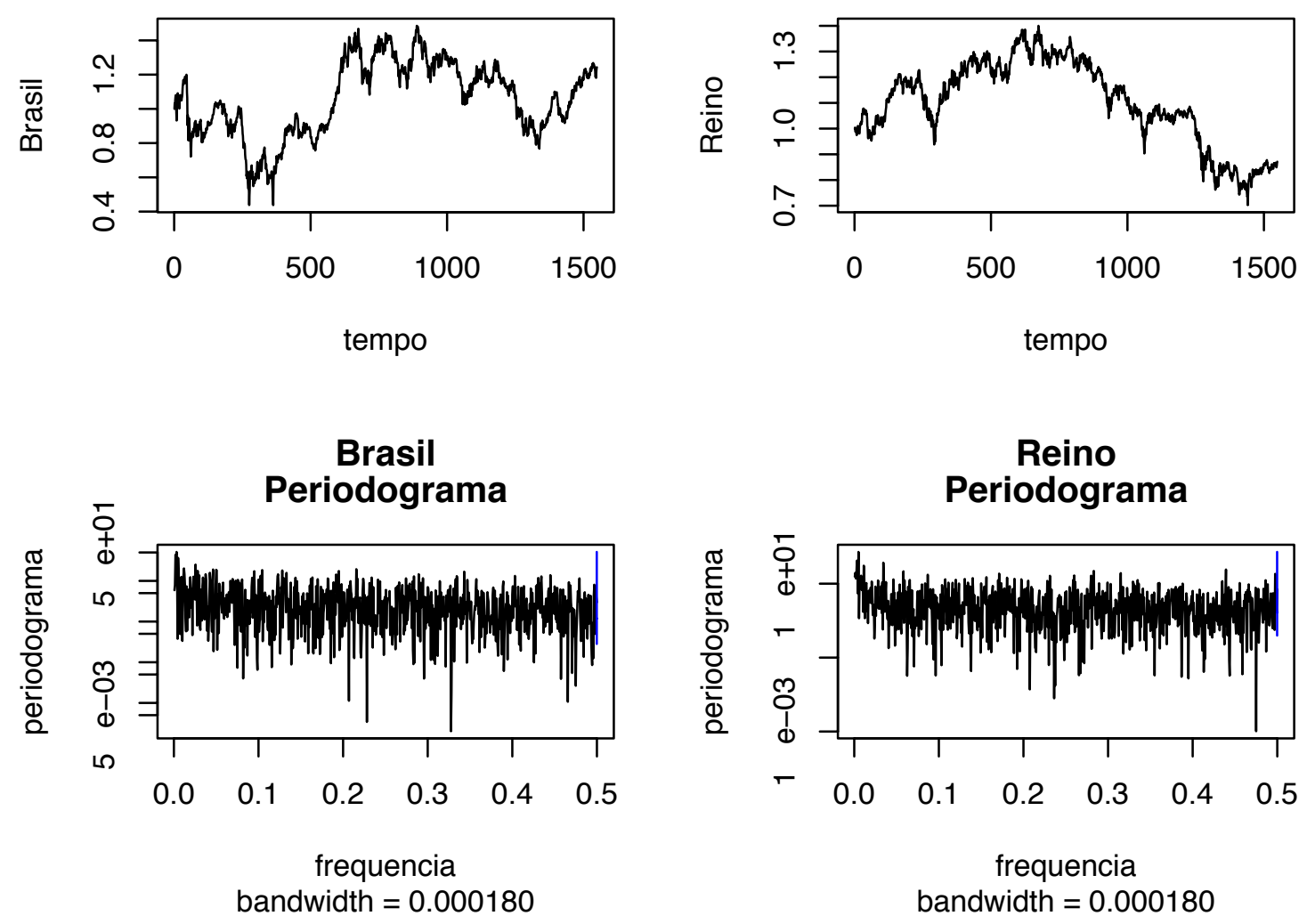

Figura 6.8: Índices das Bolsas de Valores e Periodograma das séries de volatilidade.

a função de autocorrelação cruzada decai rapidamente, sugerindo que não existe memória longa compartilhada neste caso. As estimativas do parâmetro de memória longa estão nas Tabelas 6.11 e 6.12 .

Os estimadores também indicam que as séries de volatilidade possuem persistência. Os resultados do teste MLC $(j=3, h=5)$ podem ser encontrados na Tabela 6.13. Note que as estatísticas $T$ e $T^{*}$ são significativas para $\rho_{1}^{2}$ e $\rho_{2}^{2}$ indicando que as séries não possuem memória longa compartilhada. 
Brasil

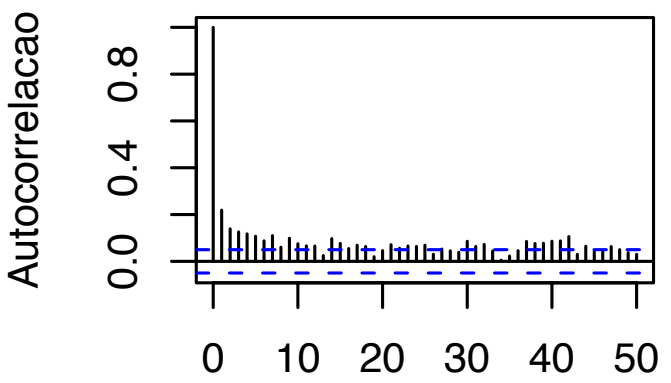

Lag
Reino \& Brasil

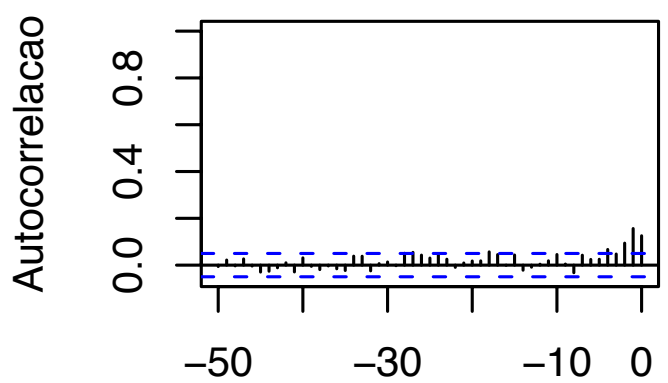

Lag

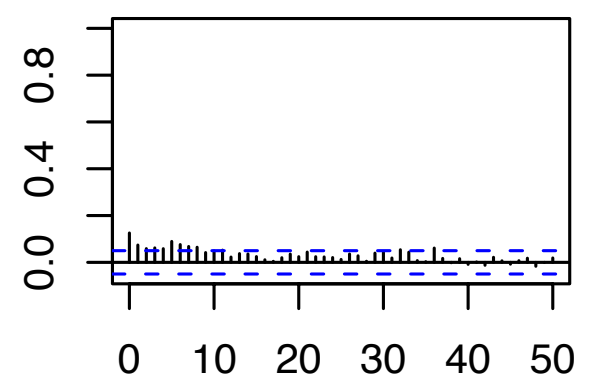

Lag
Brasil \& Reino

Reino

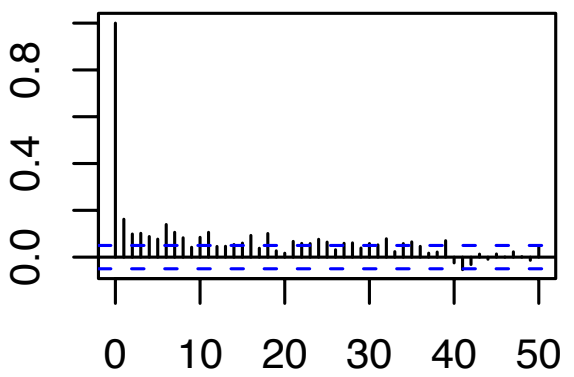

Lag

Figura 6.9: Funções de Autocorrelação e Autocorrelação Cruzada das Séries de Volatilidade.

\begin{tabular}{|c|ccc|}
\hline Brasil & d & desv.pad. & p-valor \\
\hline$\hat{d}_{T W}$ & 0,213 & 0,038 & 0,000 \\
$\hat{d}_{S}$ & 0,305 & 0,082 & 0,001 \\
$\hat{d}_{G P H}$ & 0,290 & 0,147 & 0,055 \\
\hline
\end{tabular}

Tabela 6.11: Estimação de $d$ da série de volatilidade da bolsa do Brasil. 


\begin{tabular}{|c|ccc|}
\hline Reino Unido & d & desv.pad. & p-valor \\
\hline$\hat{d}_{T W}$ & 0,275 & 0,038 & 0,000 \\
$\hat{d}_{S}$ & 0,400 & 0,082 & 0,000 \\
$\hat{d}_{G P H}$ & 0,427 & 0,090 & 0,000 \\
\hline
\end{tabular}

Tabela 6.12: Estimação de $d$ da série de volatilidade da bolsa do Reino Unido.

Dessa forma, o teste indica que as séries de volatilidade não possuem memória longa

\begin{tabular}{|c|cc|cc|}
\hline & $T$ & p-valor & $T^{*}$ & p-valor \\
\hline$\rho_{1}^{2}$ & 23,973 & 0,004 & 21,003 & 0,013 \\
$\rho_{2}^{2}$ & 93,995 & 0,000 & 61,975 & 0,000 \\
\hline
\end{tabular}

Tabela 6.13: Estatísticas $T$ e $T^{*}$ para o teste de memória longa compartilhada da volatilidade das bolsas do Brasil e Reino Unido.

compartilhada. A Figura 6.10 descreve a série de combinação linear $\left(y^{*}\right)$ correspondente à $\rho_{1}^{2}$, e sua função de autocorrelacao. A combinação linear é dada pelo vetor $(-0,530 ; 0,848)$, onde a primeira coordenada refere-se a série brasileira

Através da função de autocorrelação, observa-se que a persistência ainda permanece. As estimativas do parâmetro $d$ estão na Tabela 6.14

Os estimadores indicam que a série possui persistência. Portanto, rejeita-se a hipótese

\begin{tabular}{|c|ccc|}
\hline $\mathbf{y}$ & $\mathbf{d}$ & desv.pad. & p-valor \\
\hline$\hat{d}_{T W}$ & 0,190 & 0,038 & 0,000 \\
$\hat{d}_{S}$ & 0,316 & 0,082 & 0,000 \\
$\hat{d}_{G P H}$ & 0,387 & 0,115 & 0,002 \\
\hline
\end{tabular}

Tabela 6.14: Estimação de $d$ da combinação linear y.

de que a volatilidade da bolsa do Brasil e Reino Unido possuem memória longa compartilhada. 

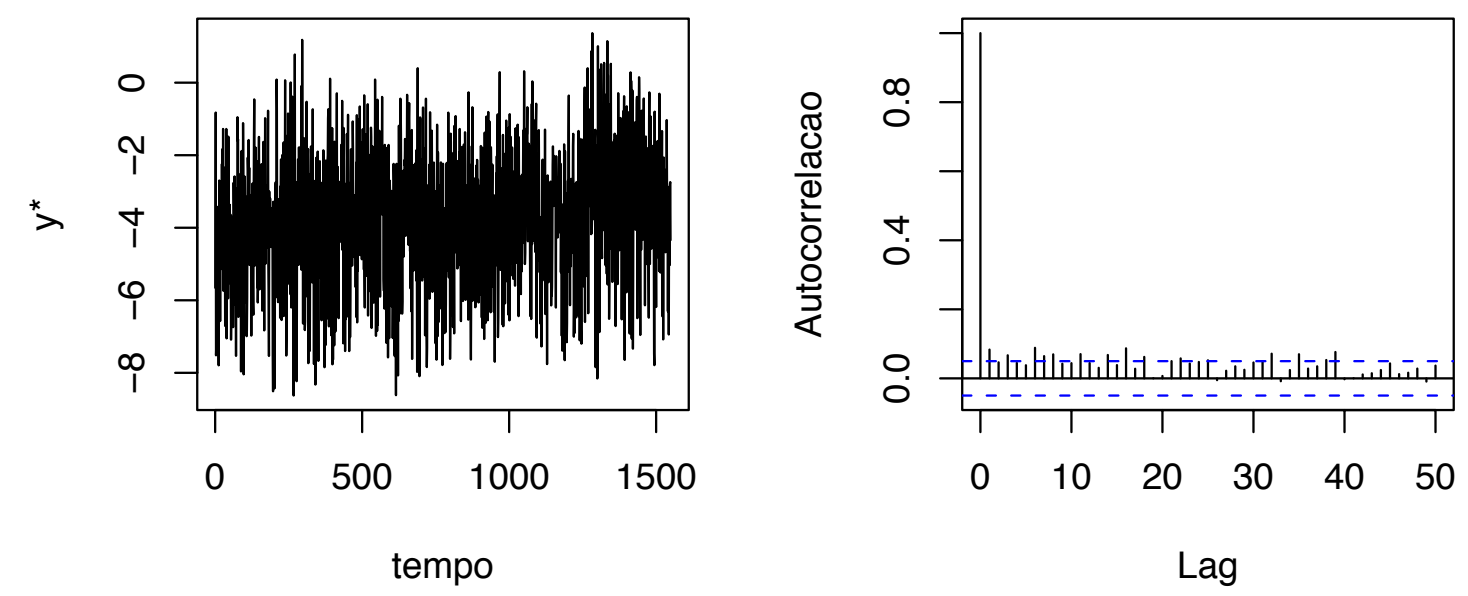

Figura 6.10: Combinação Linear das Séries de Volatilidade e sua Função de Autocorrelação. 


\section{CAPÍTULO 7}

\section{Conclusões e Resultados}

Os modelos de memória longa são freqüentemente utilizados, no estudo de séries climatológicas, hidrológicas, e atualmente, seus usos vêm se destacando na modelagem de séries financeiras. Observa-se que modelos de memória longa são adequados principalmente para a volatilidade de ativos financeiros. Estudos das características e da estrutura de dependência de séries multivariadas persistentes atualmente estão tornando-se relevantes para a identificação de componentes comuns às séries. O estudo aprofundado de métodos para identificação de séries multivariadas persistentes foi o objetivo deste trabalho, e diversas características interessantes puderam ser detectadas.

Tendo em vista todos os aspectos apresentados neste trabalho, concluimos primeiramente que o desempenho dos estimadores do parâmetro de persistência, no contexto de processos ARFIMA + ARMA, está muito relacionada com os coeficientes autoregressivos e de médias móveis, tanto do termo de memória longa, quanto ao termo de memória curta. Em geral, o estimador de Geweke e Porter-Hudak $\left(\hat{d}_{G P H}\right)$ é o que apresenta maior variabilidade, sendo portanto pouco eficiente para a identificação de processos com memória longa. Devido ao viés causado por variações nos parâmetros $\phi$ e $\theta$, o estimador truncado de Whittle $(\hat{d})$ não apresenta um bom desempenho prático, pois se o utilizarmos para um teste de persistência $\left(H_{0}: d=0\right.$ vs $H_{A}: d \neq 0$ ), o teste apresenta grande erro do tipo I no caso de processos que não são ruído branco. Concluimos que o estimador do periodograma suavizado, utilizando a janela de Bartlett-Priestley é o mais indicado para a estimação do parâmetro de memória longa, e conseqüentemente para a identificação de processos persistentes.

Além disso, concluimos também que o teste de memória longa compartilhada através 
da análise de correlação canônica (MLC) proposto por Ray e Tsay (1997) apresenta-se adequado e eficiente. No entanto, verifica-se que seu desempenho também é afetado por variações nos coeficientes autoregressivos e de médias móveis, tanto do termo de memória longa, quanto no termo de memória curta. A estatística $T^{*}$ mostrou maior eficiência no caso autoregressivo e a estatística $T$ mostrou-se mais adequada no caso de médias móveis. Além disso, verificou-se que as combinações lineares das séries sugeridas pelo teste, na maior parte dos casos não possui memória longa, ressaltando a eficiência do método.

Por fim, verifica-se a eficiência e praticidade dos métodos em aplicações a dados reais descritos no capítulo 6, tanto para dados da área de climatologia como para dados financeiros. 
APÊNDICE A

\section{Tabelas das Simulações dos Estimadores de d}

Neste apêndice encontram-se tabelas referentes aos conjuntos de simulações 1 ao 5 . As tabelas contém a média e desvio padrão das estimativas de d. Também são descritas a proporção de rejeição da hipótese $d=0$, viés e o erro quadrático médio (EQM) de cada estimador. 


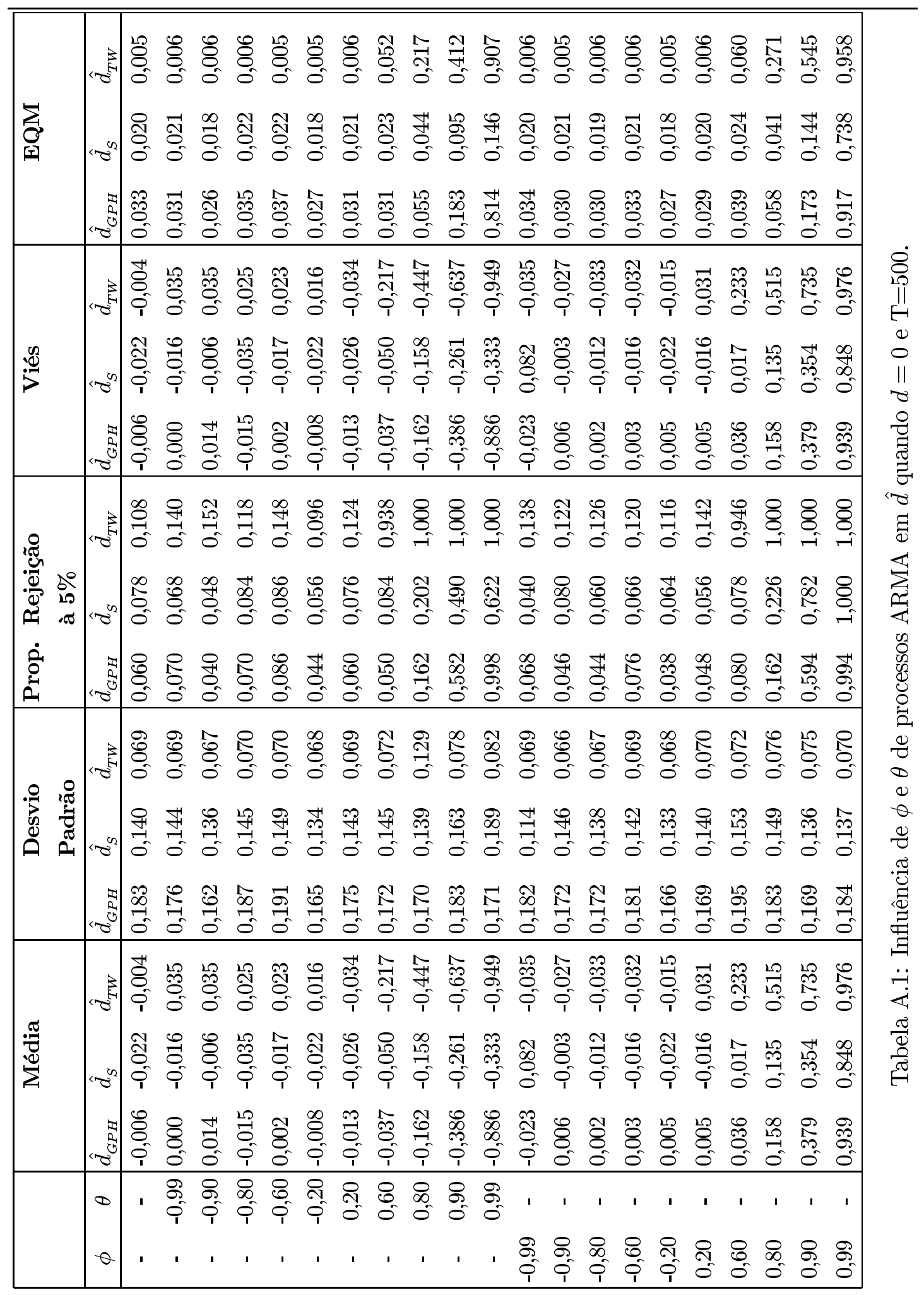




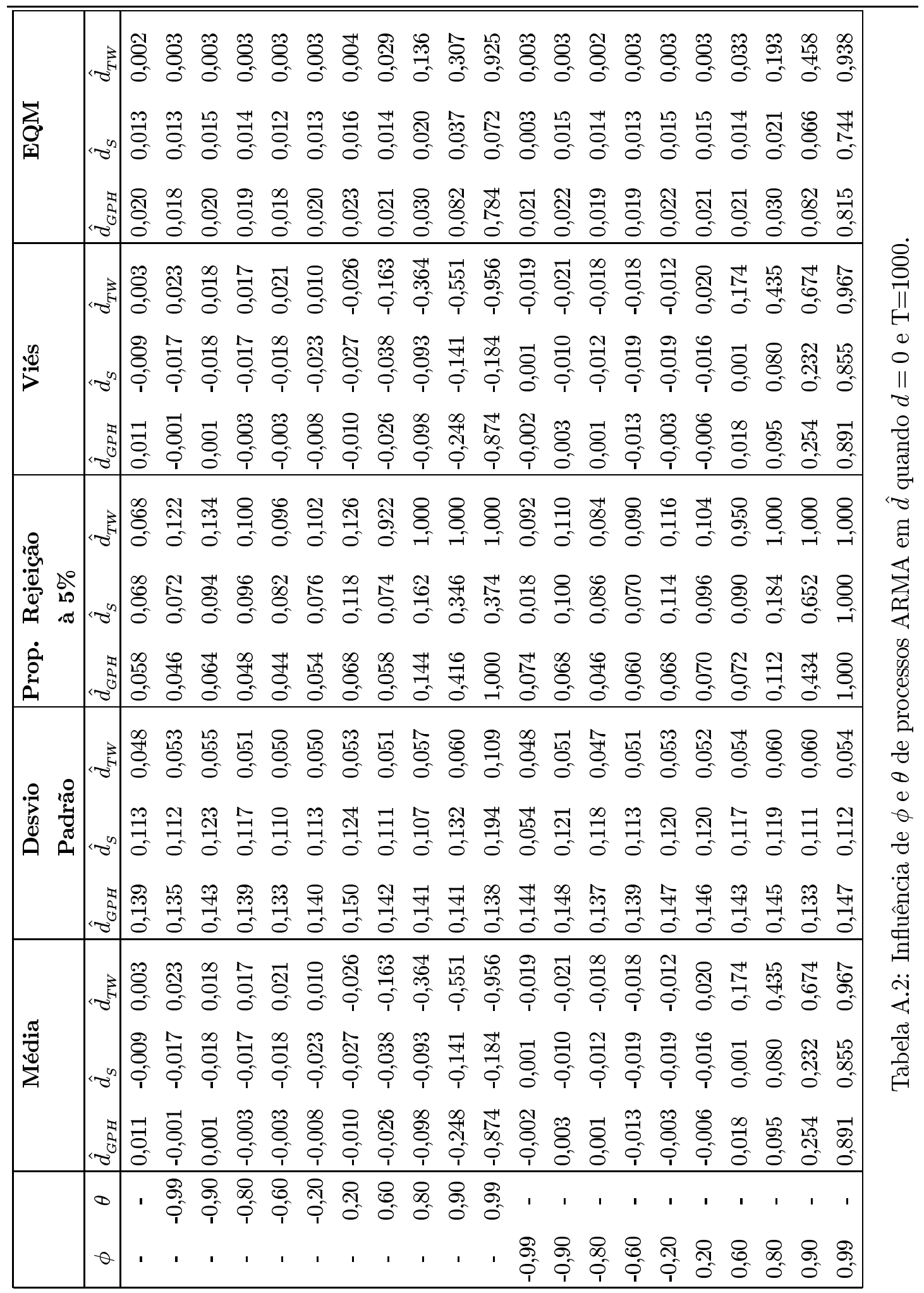




\begin{tabular}{|c|c|c|c|c|c|}
\hline & , & 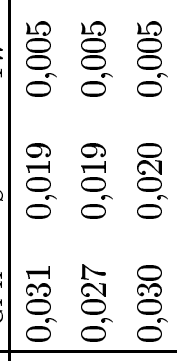 & 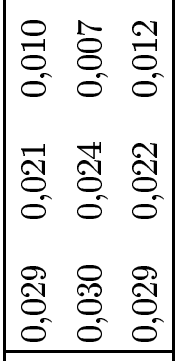 & 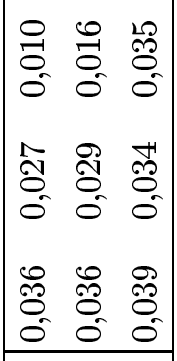 & 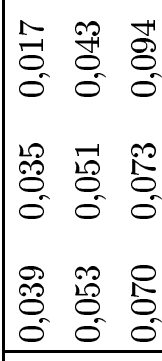 \\
\hline$\stackrel{Q}{Q}$ & $\langle 0$ & 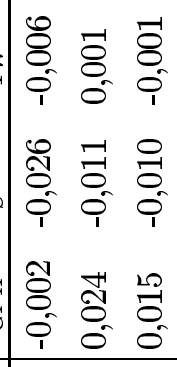 & 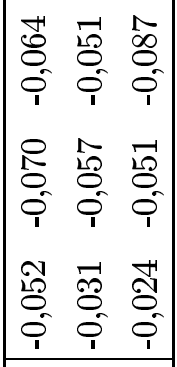 & 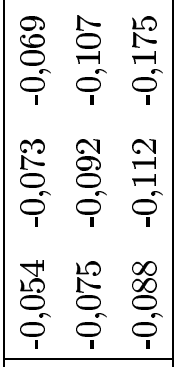 & 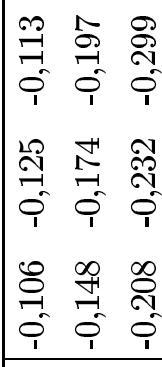 \\
\hline 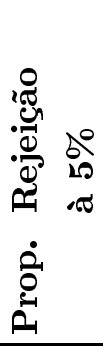 & $\begin{array}{r}\frac{\pi}{8} \\
0 \\
0 \\
0\end{array}$ & 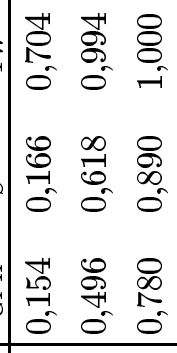 & 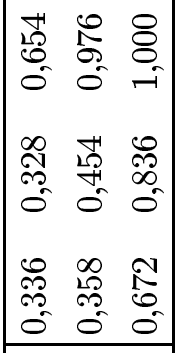 & 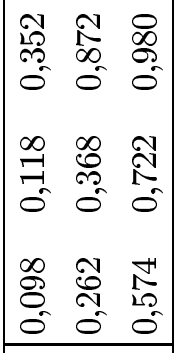 & 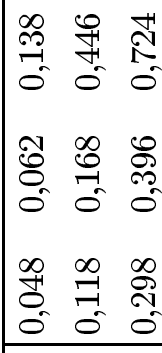 \\
\hline 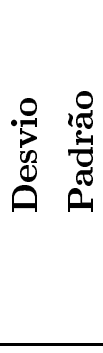 & 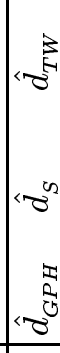 & 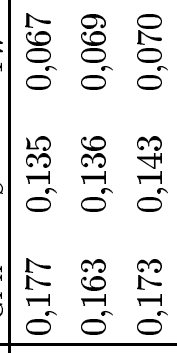 & 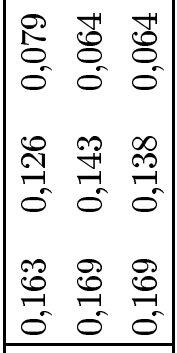 & 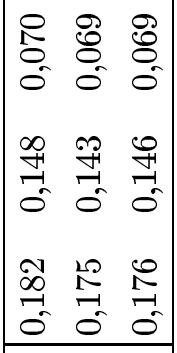 & 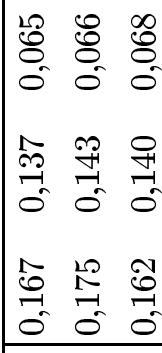 \\
\hline |: & $x^{n}$ & 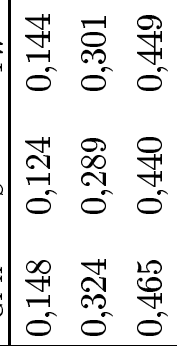 & 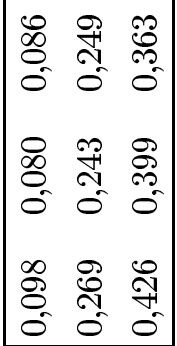 & 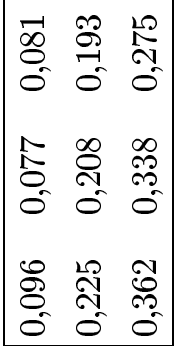 & 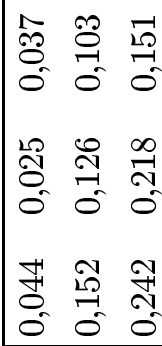 \\
\hline & $\tau$ & $\begin{array}{ccc}12 & 0 & 12 \\
9 & 09 & 7 \\
0 & 0 & 0 \\
\end{array}$ & $\begin{array}{|lll|}10 & 0 & 120 \\
9 & 0 & 9 \\
0 & 0 & 0 \\
\end{array}$ & \begin{tabular}{|lll|}
10 & 0 & 128 \\
\hdashline & 0 & 9 \\
0 & 0 & 0 \\
\end{tabular} & \begin{tabular}{|lll}
10 & 8 & 19 \\
0 & 0 & 9 \\
0 & 0 & 0 \\
\end{tabular} \\
\hline & 10 & $\stackrel{0}{0}$ & $\stackrel{0}{0}$ & $\stackrel{\circ}{\rightarrow}$ & - \\
\hline
\end{tabular}

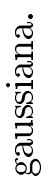




\begin{tabular}{|c|c|c|c|c|c|}
\hline 离 & $\begin{array}{l}3 \\
0 \\
0 \\
0 \\
0\end{array}$ & 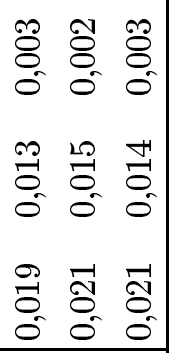 & 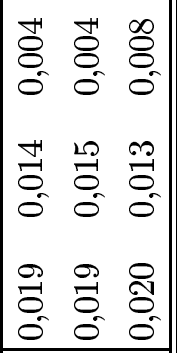 & 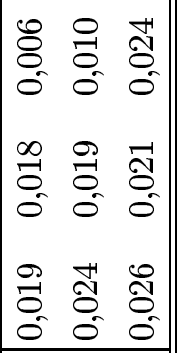 & 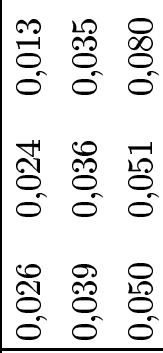 \\
\hline 选 & $\begin{array}{l}1 \\
0 \\
0 \\
0 \\
0\end{array}$ & 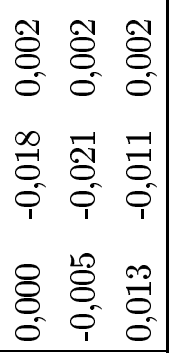 & 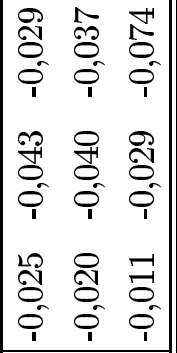 & 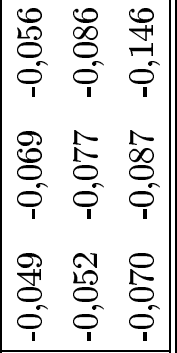 & 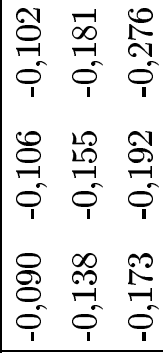 \\
\hline 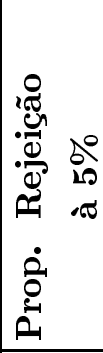 & $\begin{array}{r}7 \\
0 \\
0 \\
0 \\
0\end{array}$ & 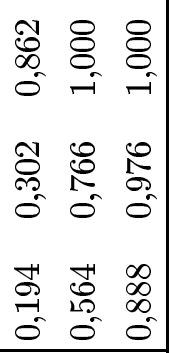 & 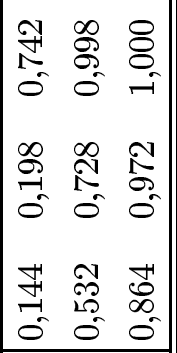 & 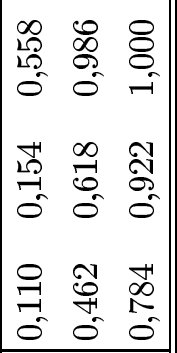 & 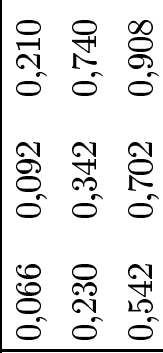 \\
\hline 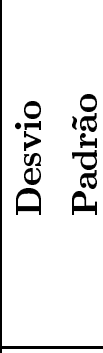 & $\begin{array}{r}2 \\
0 \\
< \\
0\end{array}$ & 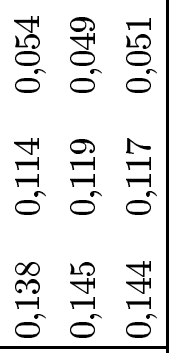 & 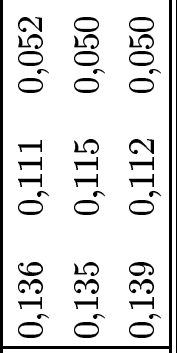 & 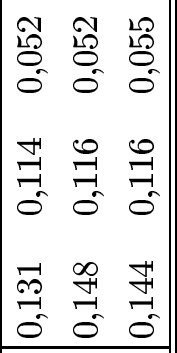 & 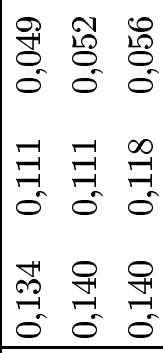 \\
\hline 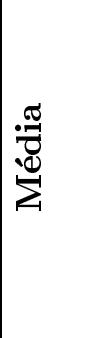 & $\mid \overrightarrow{\mid l}$ & 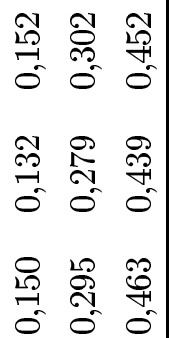 & 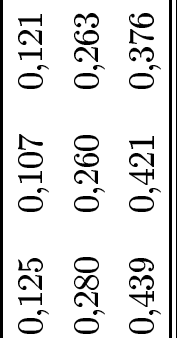 & 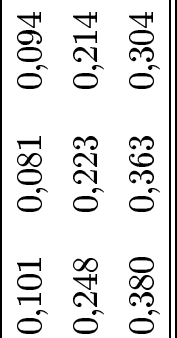 & 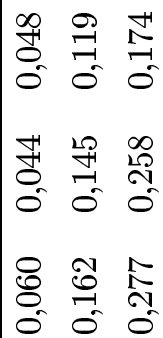 \\
\hline & $\nabla$ & 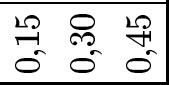 & 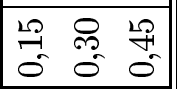 & 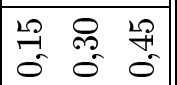 & 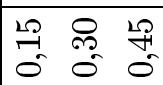 \\
\hline & Q & $\stackrel{\circ}{\circ}$ & $\ddot{0}$ & $\stackrel{\circ}{-}$ & \\
\hline
\end{tabular}




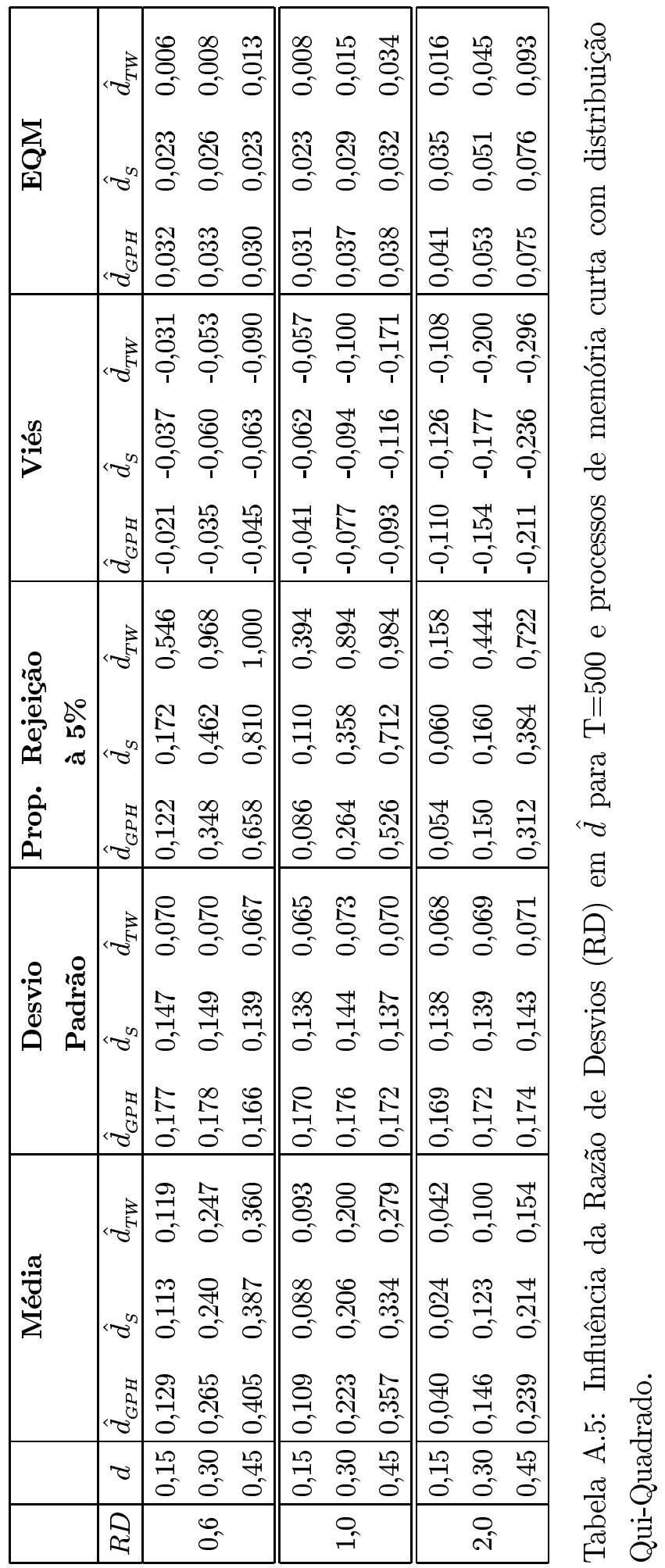




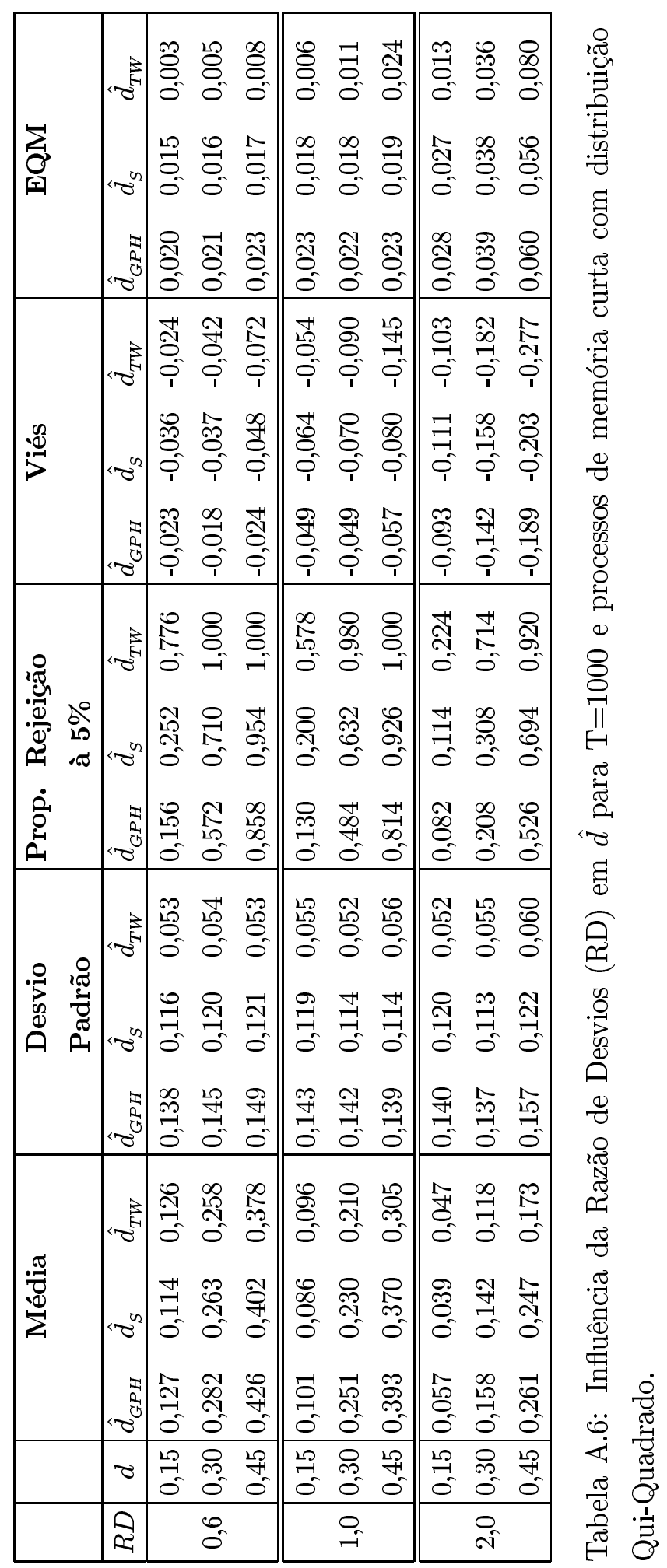




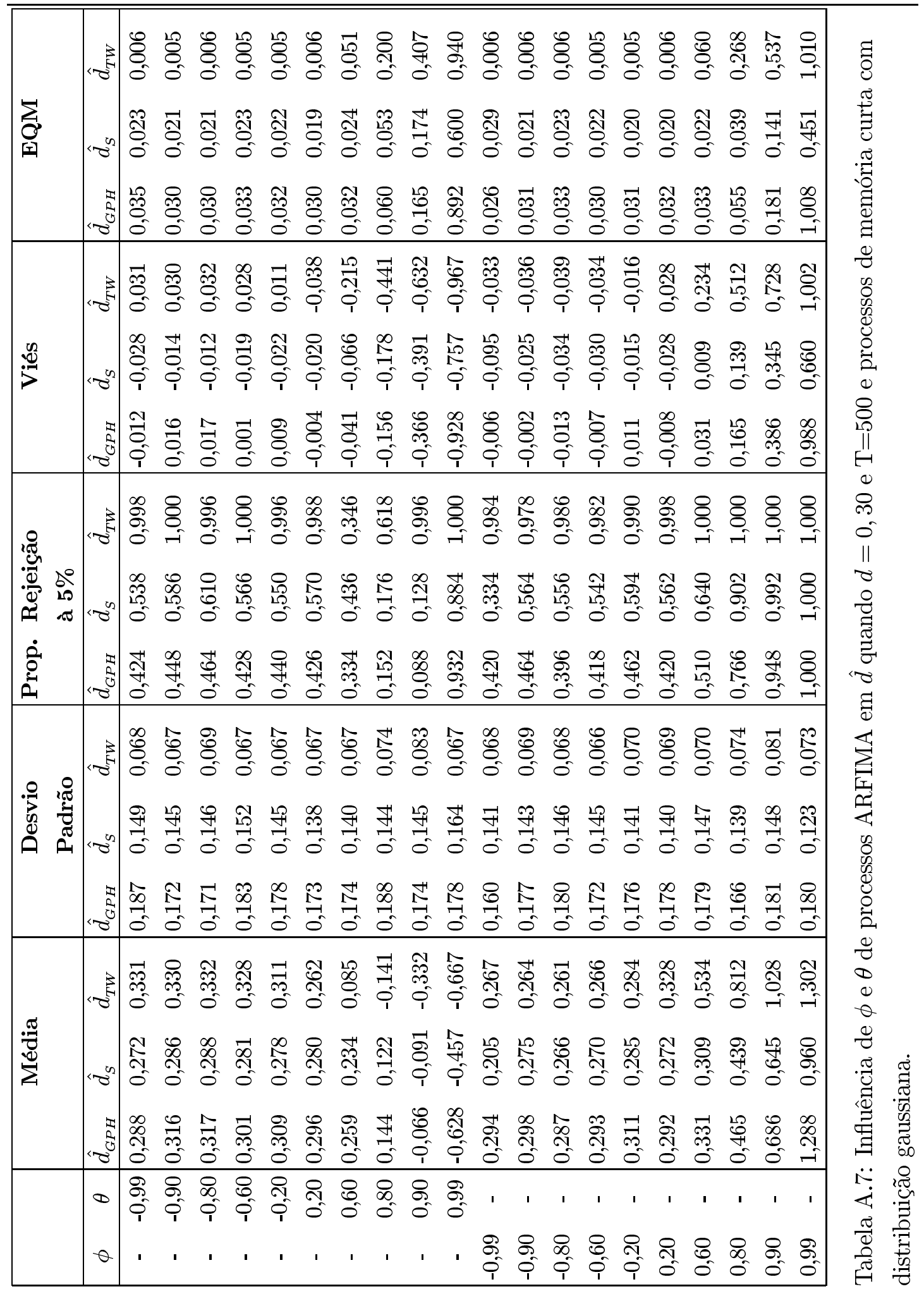




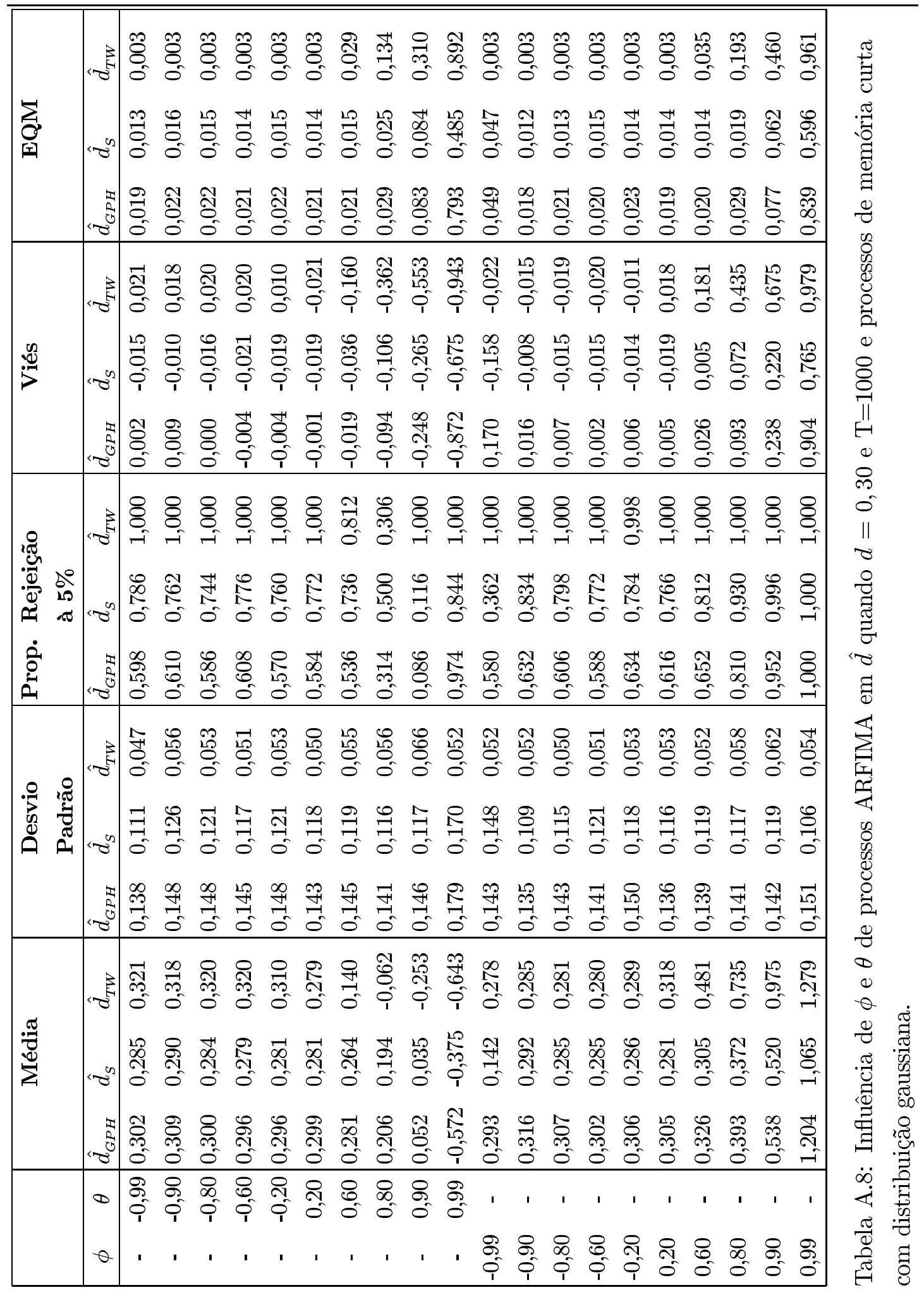




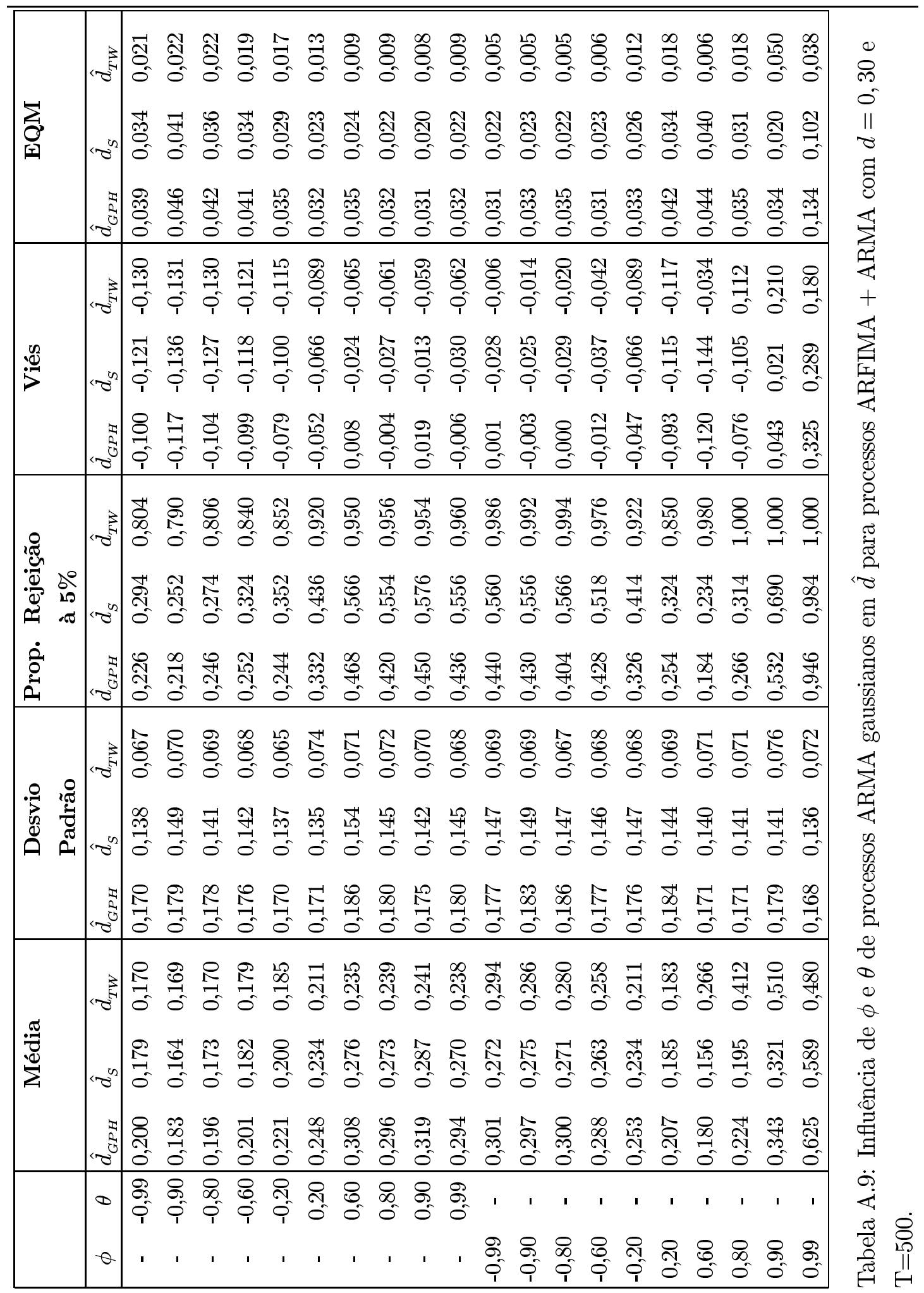




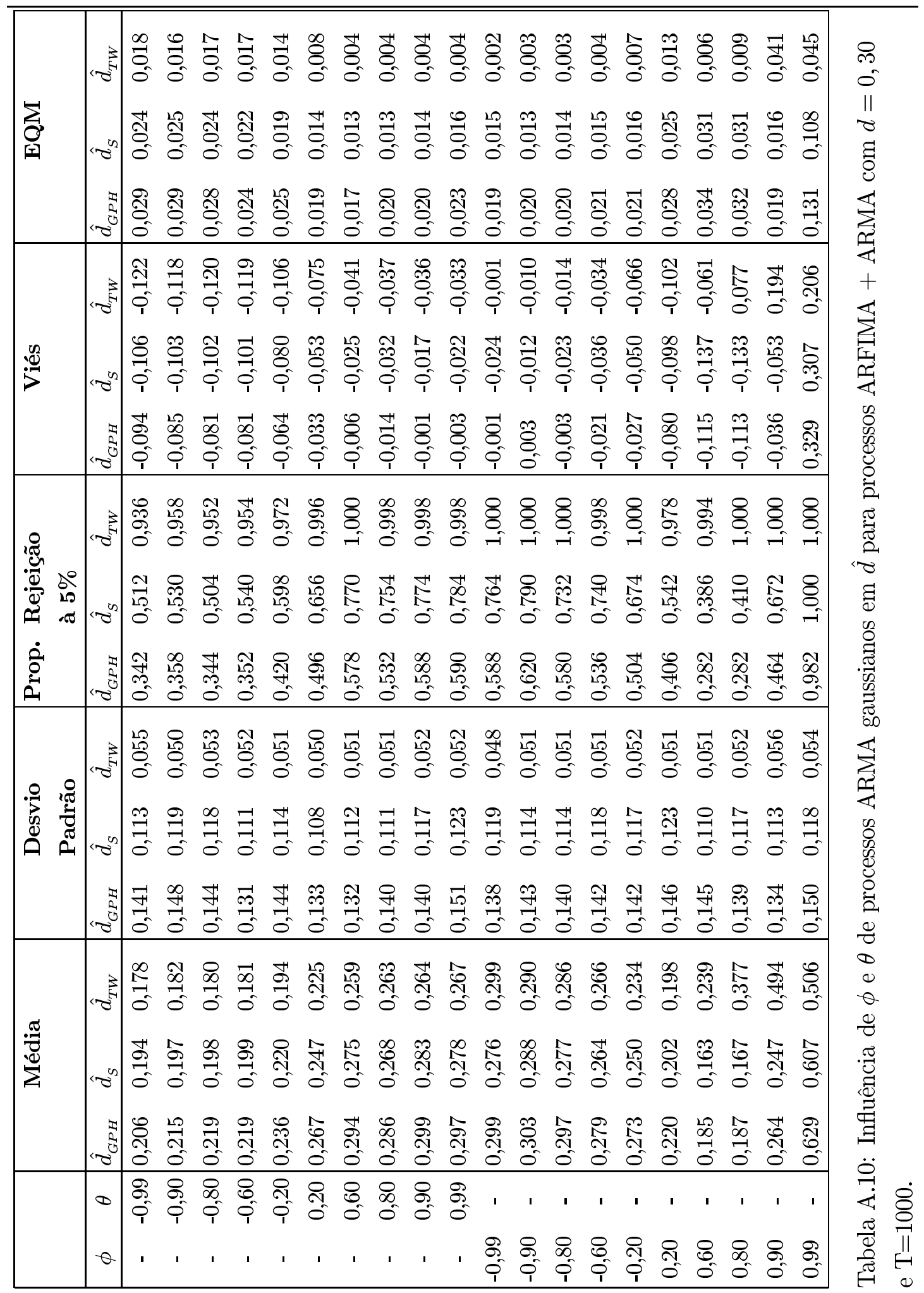




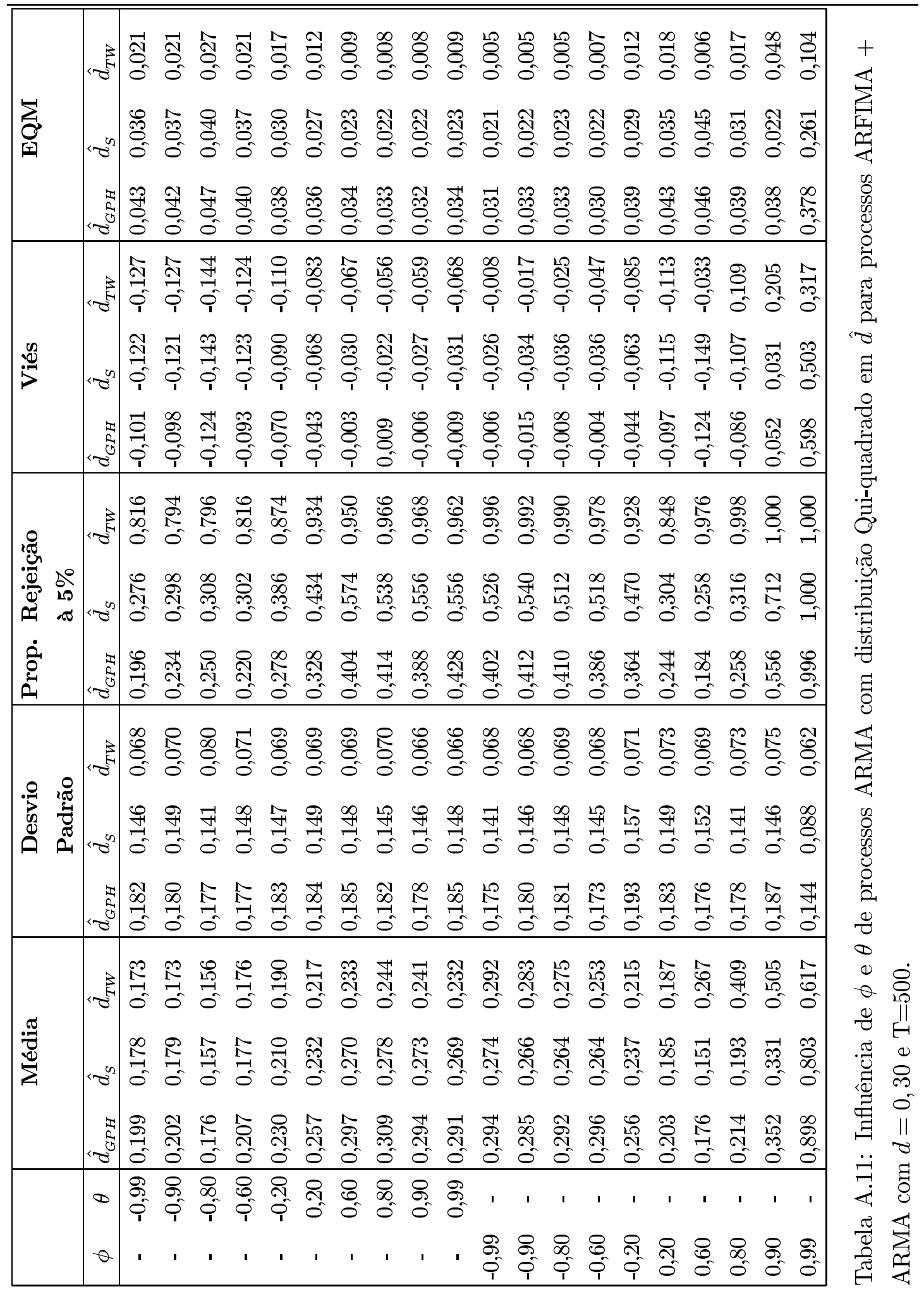




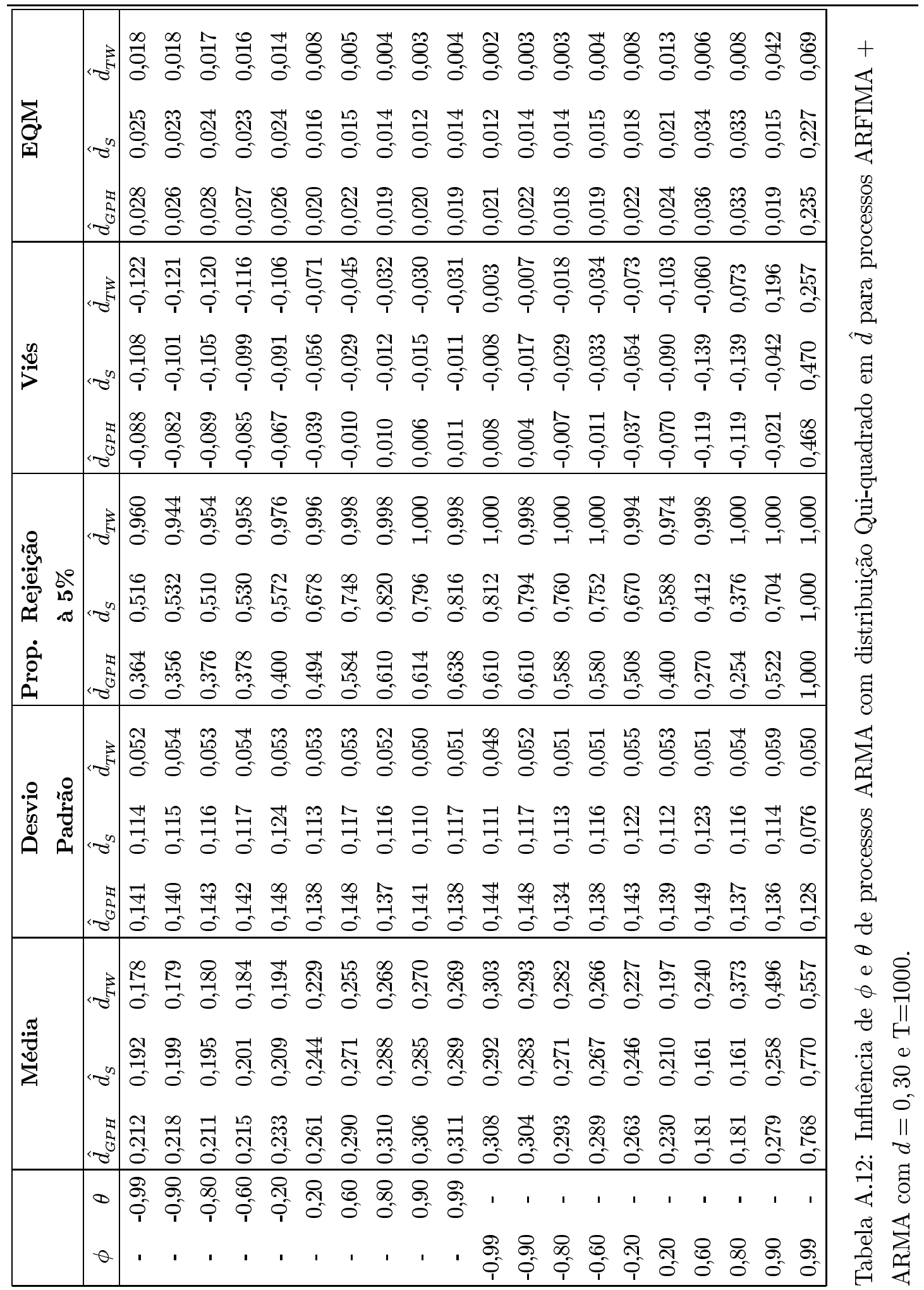


APÊNDICE B

\section{Tabelas das Simulações do Teste MLC}

Neste apêndice encontram-se tabelas referentes aos conjuntos de simulações de 5 a 7 , ou seja, das simulações do teste de memória longa compartilhada através da análise de correlação canônica. As tabelas contém os quantis observados das estatísticas $T$ e $T^{*}$ e os quantis esperados da distribuição qui-quadrado com os devidos graus de liberdade, para diversos valores de $\mathrm{h}$ e $\mathrm{j}$. Também são descritas a proporção do número de vezes que a hipótese de memória longa compartilhada é rejeitada utilizando cada uma das estatísticas, e a proporção do número de vezes em a hipótese de que a combinação linear $y^{*}$ possui memória curta é rejeitada. 


\begin{tabular}{|l|l|lllll|l|}
\hline \multirow{2}{*}{} & & \multicolumn{5}{|c|}{ Quantil } & Prop. \\
\cline { 3 - 7 } & & $\mathbf{0 , 5}$ & $\mathbf{0 , 7 5}$ & $\mathbf{0 , 9 0}$ & $\mathbf{0 , 9 5}$ & $\mathbf{0 , 9 9}$ & rej(5\%) \\
\hline \multirow{3}{*}{$\mathrm{h}=5$} & $\mathrm{~T}$ & 8,506 & 11,054 & 14,362 & 15,981 & 19,382 & 0,038 \\
$\mathrm{j}=3$ & $T^{*}$ & 8,465 & 10,967 & 14,540 & 16,141 & 19,343 & 0,038 \\
& $\chi^{2}$ & 8,343 & 11,389 & 14,684 & 16,919 & 21,666 & \\
& $\hat{d}_{T W}$ & & & & & & 0,074 \\
\hline & $\mathrm{T}$ & 8,045 & 10,812 & 14,395 & 16,460 & 20,970 & 0,046 \\
$\mathrm{~h}=5$ & $T^{*}$ & 7,931 & 10,826 & 14,437 & 16,430 & 20,858 & 0,044 \\
$\mathrm{j}=5$ & $\chi^{2}$ & 8,343 & 11,389 & 14,684 & 16,919 & 21,666 & \\
& $\hat{d}_{T W}$ & & & & & & 0,096 \\
\hline \multirow{3}{*}{$\mathrm{h}=10$} & $\mathrm{~T}$ & $\mathbf{1 7 , 9 5 7}$ & 22,172 & 26,602 & 28,534 & 37,317 & 0,034 \\
$\mathrm{j}=5$ & $T^{*}$ & 17,875 & 21,854 & 26,120 & 28,339 & 36,621 & 0,032 \\
& $\chi^{2}$ & 18,338 & 22,718 & 27,204 & 30,144 & 36,191 & \\
& $\hat{d}_{T W}$ & & & & & & 0,084 \\
\hline
\end{tabular}

Tabela B.1: Influência dos parâmetro h e j no Teste MLC para Erros com Distribuição Gaussiana.

\begin{tabular}{|l|l|lllll|l|}
\hline & & \multicolumn{5}{|c|}{ Quantil } & Prop. \\
\cline { 3 - 7 } & & $\mathbf{0 , 5}$ & $\mathbf{0 , 7 5}$ & $\mathbf{0 , 9 0}$ & $\mathbf{0 , 9 5}$ & $\mathbf{0 , 9 9}$ & rej(5\%) \\
\hline & $\mathrm{T}$ & 7,804 & 10,749 & 13,358 & 15,912 & 22,101 & 0,048 \\
$\mathrm{~h}=5$ & $T^{*}$ & 7,773 & 10,710 & 13,274 & 15,483 & 22,051 & 0,046 \\
$\mathrm{j}=3$ & $\chi^{2}$ & 8,343 & 11,389 & 14,684 & 16,919 & 21,666 & \\
& $\hat{d}_{T W}$ & & & & & & 0,092 \\
\hline & $\mathrm{T}$ & 8,112 & 11,535 & 14,845 & 16,698 & 20,607 & 0,046 \\
$\mathrm{~h}=5$ & $T^{*}$ & 8,036 & 11,330 & 14,453 & 16,674 & 20,083 & 0,044 \\
$\mathrm{j}=5$ & $\chi^{2}$ & 8,343 & 11,389 & 14,684 & 16,919 & 21,666 & \\
& $\hat{d}_{T W}$ & & & & & & 0,084 \\
\hline \multirow{3}{*}{$\mathrm{h}=10$} & $\mathrm{~T}$ & 17,299 & 21,873 & 26,927 & 28,880 & 33,070 & 0,036 \\
$\mathrm{j}=5$ & $T^{*}$ & 17,204 & 21,892 & 26,517 & 28,715 & 33,039 & 0,028 \\
& $\chi^{2}$ & 18,338 & 22,718 & 27,204 & 30,144 & 36,191 & \\
& $\hat{d}_{T W}$ & & & & & & 0,088 \\
\hline
\end{tabular}

Tabela B.2: Influência dos parâmetro h e j no Teste MLC para Erros com Distribuição Qui-quadrado. 


\begin{tabular}{|c|c|c|c|c|c|c|c|c|}
\hline & & & \multicolumn{5}{|c|}{ Quantil } & \multirow{2}{*}{$\begin{array}{l}\text { Prop. } \\
\text { rej(5\%) }\end{array}$} \\
\hline & & & 0,5 & 0,75 & 0,90 & 0,95 & 0,99 & \\
\hline \multirow{12}{*}{$\phi=-0,6$} & & $\overline{\mathrm{T}}$ & 8,114 & 11,262 & 14,254 & 16,778 & 20,866 & 0,046 \\
\hline & $\mathrm{h}=5$ & $T^{*}$ & 8,077 & 11,212 & 14,147 & 16,777 & 20,261 & 0,048 \\
\hline & $\mathrm{j}=3$ & $\chi^{2}$ & 8,343 & 11,389 & 14,684 & 16,919 & 21,666 & \\
\hline & & $\hat{d}_{T W}$ & & & & & & 0,084 \\
\hline & & $T$ & 7,495 & 10,508 & 13,477 & 15,785 & 20,134 & 0,032 \\
\hline & $\mathrm{h}=5$ & $T^{*}$ & 7,432 & 10,536 & 13,184 & 15,429 & 18,271 & 0,028 \\
\hline & $\mathrm{j}=5$ & $\chi^{2}$ & 8,343 & 11,389 & 14,684 & 16,919 & 21,666 & \\
\hline & & & & & & & & 0,136 \\
\hline & & 1 & 17,610 & 21,197 & 25,328 & 29,278 & 32,195 & 0,042 \\
\hline & $\mathrm{h}=10$ & $T^{*}$ & 17,323 & 21,134 & 25,258 & 28,056 & 31,670 & 0,034 \\
\hline & $\mathrm{j}=5$ & $\chi^{2}$ & 18,338 & 22,718 & 27,204 & 30,144 & 36,191 & \\
\hline & & $\hat{d}_{T W}$ & & & & & & 0,138 \\
\hline \multirow{12}{*}{$\phi=-0,2$} & & $\overline{\mathrm{T}}$ & 8,304 & 11,538 & 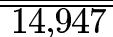 & 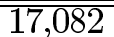 & 20,084 & 0,054 \\
\hline & $h=5$ & $T^{*}$ & 8,161 & 11,476 & 14,865 & 17,082 & 20,394 & 0,054 \\
\hline & $j=3$ & $\chi^{2}$ & 8,343 & 11,389 & 14,684 & 16,919 & 21,666 & \\
\hline & & $\hat{d}_{T w}$ & & & & & & 0,078 \\
\hline & & $T$ & 8,192 & 11,213 & 14,473 & 17,017 & 22,504 & 0,056 \\
\hline & $\mathrm{h}=5$ & $T^{*}$ & 8,199 & 11,119 & 14,365 & 16,496 & 21,624 & 0,048 \\
\hline & $\mathrm{j}=5$ & $\chi^{2}$ & 8,343 & 11,389 & 14,684 & 16,919 & 21,666 & \\
\hline & & $\hat{d}_{T W}$ & & & & & & 0,086 \\
\hline & & $T$ & 18,262 & 22,660 & 27,579 & 30,216 & 38,404 & 0,052 \\
\hline & $\mathrm{h}=10$ & $T^{*}$ & 17,963 & 22,250 & 27,187 & 29,748 & 37,890 & 0,044 \\
\hline & $\mathrm{j}=5$ & $\chi^{2}$ & 18,338 & 22,718 & 27,204 & 30,144 & 36,191 & \\
\hline & & $\hat{d}_{T W}$ & & & & & & 0,112 \\
\hline \multirow{12}{*}{$\phi=0,2$} & & $\mathrm{~T}$ & 8,515 & 11,590 & $\overline{15,323}$ & $\overline{17,439}$ & 20,249 & 0,066 \\
\hline & $\mathrm{h}=5$ & $T^{*}$ & 8,456 & 11,562 & 15,570 & 17,158 & 20,387 & 0,064 \\
\hline & $\mathrm{j}=3$ & $\chi^{2}$ & 8,343 & 11,389 & 14,684 & 16,919 & 21,666 & \\
\hline & & $\hat{d}_{T W}$ & & & & & & 0,106 \\
\hline & & $T$ & 8,422 & 11,477 & 14,728 & 17,323 & 22,171 & 0,054 \\
\hline & $\mathrm{h}=5$ & $T^{*}$ & 8,352 & 11,356 & 14,671 & 17,157 & 21,540 & 0,052 \\
\hline & $\mathrm{j}=5$ & $\chi^{2}$ & 8,343 & 11,389 & 14,684 & 16,919 & 21,666 & \\
\hline & & $\hat{d}_{T W}$ & & & & & & 0,086 \\
\hline & & $T$ & 18,617 & 22,074 & 26,635 & 30,126 & 35,125 & 0,050 \\
\hline & $\mathrm{h}=10$ & $T^{*}$ & 18,304 & 21,900 & 26,382 & 29,910 & 34,631 & 0,044 \\
\hline & $\mathrm{j}=5$ & $\chi^{2}$ & 18,338 & 22,718 & 27,204 & 30,144 & 36,191 & \\
\hline & & $\hat{d}_{T W}$ & & & & & & 0,104 \\
\hline \multirow{12}{*}{$\phi=0,6$} & & $\overline{\mathrm{T}}$ & 8,213 & 10,880 & 13,945 & $\overline{16,198}$ & $\overline{21,806}$ & 0,032 \\
\hline & $\mathrm{h}=5$ & $T^{*}$ & 8,232 & 10,810 & 13,878 & 16,407 & 21,691 & 0,032 \\
\hline & $\mathrm{j}=3$ & $\chi^{2}$ & 8,343 & 11,389 & 14,684 & 16,919 & 21,666 & \\
\hline & & $\hat{d}_{T W}$ & & & & & & 0,078 \\
\hline & & $T^{N}$ & 8,059 & 11,111 & 14,139 & 17,418 & 21,307 & 0,058 \\
\hline & $\mathrm{h}=5$ & $T^{*}$ & 8,063 & 11,064 & 14,084 & 17,467 & 21,369 & 0,054 \\
\hline & $\mathrm{j}=5$ & $\chi^{2}$ & 8,343 & 11,389 & 14,684 & 16,919 & 21,666 & \\
\hline & & $\hat{d}_{T W}$ & & & & & & 0,084 \\
\hline & & $T$ & 17,801 & 21,955 & 26,452 & 29,410 & 35,537 & 0,042 \\
\hline & $\mathrm{h}=10$ & $T^{*}$ & 17,618 & 21,884 & 26,118 & 29,512 & 34,270 & 0,038 \\
\hline & $\mathrm{j}=5$ & $\chi^{2}$ & 18,338 & 22,718 & 27,204 & 30,144 & 36,191 & \\
\hline & & $\hat{d}_{T W}$ & & & & & & 0,088 \\
\hline
\end{tabular}

Tabela B.3: Influência do Coeficiente Autoregressivo $(\phi)$ do Processo ARFIMA no Teste MLC. 


\begin{tabular}{|c|c|c|c|c|c|c|c|c|}
\hline & & & \multicolumn{5}{|c|}{ Quantil } & \multirow{2}{*}{$\begin{array}{l}\text { Prop. } \\
\text { rej(5\%) }\end{array}$} \\
\hline & & & 0,5 & 0,75 & 0,90 & 0,95 & 0,99 & \\
\hline \multirow{12}{*}{$\theta=-0,6$} & & $\mathrm{~T}$ & 8,404 & 11,115 & 13,685 & 16,197 & 22,061 & 0,042 \\
\hline & $\mathrm{h}=5$ & $T^{*}$ & 8,454 & 11,122 & 13,699 & 16,140 & 21,804 & 0,040 \\
\hline & $\mathrm{j}=3$ & $\chi^{2}$ & 8,343 & 11,389 & 14,684 & 16,919 & 21,666 & \\
\hline & & $\hat{d}_{T W}$ & & & & & & 0,086 \\
\hline & & $T$ & 8,270 & 11,288 & 14,573 & 16,487 & 21,752 & 0,038 \\
\hline & $\mathrm{h}=5$ & $T^{*}$ & 8,224 & 11,144 & 14,638 & 16,468 & 21,770 & 0,038 \\
\hline & $\mathrm{j}=5$ & $\chi^{2}$ & 8,343 & 11,389 & 14,684 & 16,919 & 21,666 & \\
\hline & & & & & & & & 0,062 \\
\hline & & $\mathrm{T}$ & 18,249 & 22,778 & 26,567 & 29,708 & 35,999 & 0,044 \\
\hline & $\mathrm{h}=10$ & $T^{*}$ & 18,085 & 22,684 & 25,973 & 28,914 & 35,807 & 0,044 \\
\hline & $\mathrm{j}=5$ & $\chi^{2}$ & 18,338 & 22,718 & 27,204 & 30,144 & 36,191 & \\
\hline & & $\hat{d}_{T W}$ & & & & & & 0,086 \\
\hline \multirow{12}{*}{$\theta=-0,2$} & & $T$ & 8,453 & $\overline{111,112}$ & $=14,498$ & $=16,534$ & $\overline{201,023}$ & 0,042 \\
\hline & $\mathrm{h}=5$ & $T^{*}$ & 8,362 & 11,152 & 14,377 & 16,385 & 21,056 & 0,042 \\
\hline & $j=3$ & $\chi^{2}$ & 8,343 & 11,389 & 14,684 & 16,919 & 21,666 & \\
\hline & & $\hat{d}_{T W}$ & & & & & & 0,074 \\
\hline & & $T$ & 8,027 & 10,886 & 14,410 & 17,308 & 22,122 & 0,056 \\
\hline & $\mathrm{h}=5$ & $T^{*}$ & 8,013 & 10,908 & 14,257 & 17,067 & 21,655 & 0,052 \\
\hline & $\mathrm{j}=5$ & $\chi^{2}$ & 8,343 & 11,389 & 14,684 & 16,919 & 21,666 & \\
\hline & & $\hat{d}_{T W}$ & & & & & & 0,050 \\
\hline & & $T$ & 17,747 & 22,417 & 26,549 & 28,545 & 34,010 & 0,034 \\
\hline & $h=10$ & $T^{*}$ & 17,614 & 22,055 & 26,321 & 28,639 & 33,002 & 0,026 \\
\hline & $\mathrm{j}=5$ & $\chi^{2}$ & 18,338 & 22,718 & 27,204 & 30,144 & 36,191 & \\
\hline & & $\hat{d}_{T W}$ & & & & & & 0,082 \\
\hline \multirow{12}{*}{$\theta=0,2$} & & $\bar{T}$ & 8,412 & 11,245 & 14,275 & 16,124 & $\overline{19} 19,756$ & 0,034 \\
\hline & $\mathrm{h}=5$ & $T^{*}$ & 8,420 & 11,227 & 14,238 & 15,879 & 19,743 & 0,040 \\
\hline & $\mathrm{j}=3$ & $\chi^{2}$ & 8,343 & 11,389 & 14,684 & 16,919 & 21,666 & \\
\hline & & $\hat{d}_{T W}$ & & & & & & 0,084 \\
\hline & & $\mathrm{T}$ & 7,709 & 10,895 & 14,242 & 16,937 & 22,113 & 0,052 \\
\hline & $\mathrm{h}=5$ & $T^{*}$ & 7,668 & 10,794 & 14,152 & 16,701 & 22,028 & 0,046 \\
\hline & $\mathrm{j}=5$ & $\chi^{2}$ & 8,343 & 11,389 & 14,684 & 16,919 & 21,666 & \\
\hline & & $\hat{d}_{T W}$ & & & & & & 0,122 \\
\hline & & $T$ & 17,678 & 22,069 & 26,329 & 28,544 & 33,165 & 0,026 \\
\hline & $\mathrm{h}=10$ & $T^{*}$ & 17,593 & 21,816 & 26,025 & 28,554 & 32,344 & 0,026 \\
\hline & $\mathrm{j}=5$ & $\chi^{2}$ & 18,338 & 22,718 & 27,204 & 30,144 & 36,191 & \\
\hline & & $\hat{d}_{T W}$ & & & & & & 0,128 \\
\hline \multirow{12}{*}{$\theta=0,6$} & & $\mathrm{~T}$ & 6,432 & 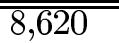 & 10,915 & 12,445 & 16,020 & 0,006 \\
\hline & $\mathrm{h}=5$ & $T^{*}$ & 6,430 & 8,523 & 10,933 & 12,527 & 15,703 & 0,006 \\
\hline & $j=3$ & $\chi^{2}$ & 8,343 & 11,389 & 14,684 & 16,919 & 21,666 & \\
\hline & & $\hat{d}_{T W}$ & & & & & & 0,108 \\
\hline & & $\mathrm{T}$ & 6,219 & 8,284 & 10,511 & 11,951 & 14,806 & 0,002 \\
\hline & $\mathrm{h}=5$ & $T^{*}$ & 6,200 & 8,213 & 10,485 & 11,893 & 14,713 & 0,004 \\
\hline & $\mathrm{j}=5$ & $\chi^{2}$ & 8,343 & 11,389 & 14,684 & 16,919 & 21,666 & \\
\hline & & $\hat{d}_{T W}$ & & & & & & 0,150 \\
\hline & & $T$ & 14,742 & 17,975 & 21,943 & 24,353 & 27,606 & 0,002 \\
\hline & $\mathrm{h}=10$ & $T^{*}$ & 14,775 & 17,830 & 21,981 & 23,959 & 27,462 & 0,002 \\
\hline & $\mathrm{j}=5$ & $\chi^{2}$ & 18,338 & 22,718 & 27,204 & 30,144 & 36,191 & \\
\hline & & $\hat{d}_{T W}$ & & & & & & 0,124 \\
\hline
\end{tabular}

Tabela B.4: Influência do Coeficiente de Médias Móveis $(\theta)$ do Processo ARFIMA no Teste MLC. 


\begin{tabular}{|c|c|c|c|c|c|c|c|c|}
\hline & & & \multicolumn{5}{|c|}{ Quantil } & \multirow{2}{*}{$\begin{array}{l}\text { Prop. } \\
\text { rej(5\%) }\end{array}$} \\
\hline & & & 0,5 & 0,75 & 0,90 & 0,95 & 0,99 & \\
\hline \multirow{12}{*}{$\phi=-0,6$} & & $\mathrm{~T}$ & 51,500 & 62,613 & 74,392 & 87,457 & 101,442 & 0.996 \\
\hline & $h=5$ & $T^{*}$ & 29,137 & 35,642 & 43,925 & 46,927 & 56,683 & 0,942 \\
\hline & $j=3$ & $\chi^{2}$ & 8,343 & 11,389 & 14,684 & 16,919 & 21,666 & \\
\hline & & $\hat{\hat{d}}_{S}$ & & & & & & 0,288 \\
\hline & & $\mathrm{T}$ & 14,159 & 20,302 & 25,700 & 30,623 & 41,337 & 0,378 \\
\hline & $h=5$ & $T^{*}$ & 7,669 & 10,616 & 13,530 & 15,822 & 22,618 & 0,032 \\
\hline & $\mathrm{j}=5$ & $\chi^{2}$ & 8,343 & 11,389 & 14,684 & 16,919 & 21,666 & \\
\hline & & & & & & & & 0,128 \\
\hline & & $\mathrm{T}$ & 24,771 & 33,183 & 40,437 & 46,468 & 59,113 & 0,324 \\
\hline & $h=10$ & $T^{*}$ & 13,254 & 16,630 & 19,812 & 22,665 & 31,032 & 0,014 \\
\hline & $\mathrm{j}=5$ & $\chi^{2}$ & 18,338 & 22,718 & 27,204 & 30,144 & 36,191 & \\
\hline & & $\hat{\hat{d}}_{S}$ & & & & & & 0,122 \\
\hline \multirow{12}{*}{$\phi=-0,2$} & & 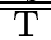 & $\overline{88,644}$ & 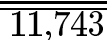 & $\overline{15,060}$ & $\overline{177,269}$ & 23,637 & 0,054 \\
\hline & $\mathrm{h}=5$ & $T^{*}$ & 7,850 & 10,609 & 13,755 & 15,940 & 19,373 & 0,032 \\
\hline & $j=3$ & $\chi^{2}$ & 8,343 & 11,389 & 14,684 & 16,919 & 21,666 & \\
\hline & & $\hat{d}_{S}$ & & & & & & 0,096 \\
\hline & & $T$ & 8,278 & 11,613 & 14,743 & 17,012 & 24,254 & 0,056 \\
\hline & $\mathrm{h}=5$ & $T^{*}$ & 7,913 & 10,491 & 12,938 & 14,765 & 20,724 & 0,032 \\
\hline & $\mathrm{j}=5$ & $\chi^{2}$ & 8,343 & 11,389 & 14,684 & 16,919 & 21,666 & \\
\hline & & $\hat{\hat{d}}_{S}$ & & & & & & 0,106 \\
\hline & & $T$ & 18,200 & 22,767 & 26,047 & 28,751 & 34,489 & 0,042 \\
\hline & $h=10$ & $T^{*}$ & 16,835 & 20,868 & 23,917 & 27,304 & 32,841 & 0,034 \\
\hline & $\mathrm{j}=5$ & $\chi^{2}$ & 18,338 & 22,718 & 27,204 & 30,144 & 36,191 & \\
\hline & & $\hat{\hat{d}}_{S}$ & & & & & & 0,108 \\
\hline \multirow{12}{*}{$\phi=0,2$} & & $\overline{\mathrm{T}}$ & 7,675 & 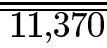 & $\overline{14,418}$ & $\overline{10,836}$ & 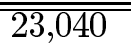 & 0,050 \\
\hline & $h=5$ & $T^{*}$ & 7.710 & 10.509 & 14.087 & 16.136 & 20.581 & 0.034 \\
\hline & $\mathrm{j}=3$ & $\chi^{2}$ & 8,343 & 11,389 & 14,684 & 16,919 & 21,666 & \\
\hline & & $\hat{d}_{S}$ & & & & & & 0,100 \\
\hline & & $\stackrel{s}{T}$ & 7,877 & 10,862 & 13,803 & 15,679 & 21,358 & 0,042 \\
\hline & $\mathrm{h}=5$ & $T^{*}$ & 7,593 & 10,625 & 13,225 & 15,314 & 18,362 & 0,028 \\
\hline & $\mathrm{j}=5$ & $\chi^{2}$ & 8,343 & 11,389 & 14,684 & 16,919 & 21,666 & \\
\hline & & $\hat{\hat{d}}_{S}$ & & & & & & 0,120 \\
\hline & & $T$ & 17,900 & 21,797 & 25,838 & 28,897 & 32,457 & 0,036 \\
\hline & $h=10$ & $T^{*}$ & 16,594 & 20,177 & 24,251 & 26,361 & 30,127 & 0,010 \\
\hline & $\mathrm{j}=5$ & $\chi^{2}$ & 18,338 & 22,718 & 27,204 & 30,144 & 36,191 & \\
\hline & & $\hat{d}_{S}$ & & & & & & 0,074 \\
\hline \multirow{12}{*}{$\phi=0,6$} & & $2 \mathrm{~T}$ & $\overline{46,112}$ & $\overline{58,603}$ & $\overline{669,563}$ & $\overline{778,548}$ & $\overline{901,114}$ & 0,996 \\
\hline & $h=5$ & $T^{*}$ & 25,648 & 30,211 & 34,862 & 37,941 & 42,408 & 0,910 \\
\hline & $j=3$ & $\chi^{2}$ & 8,343 & 11,389 & 14,684 & 16,919 & 21,666 & \\
\hline & & $\hat{d}_{S}$ & & & & & & 0,124 \\
\hline & & $\mathrm{T}$ & 11,512 & 17,412 & 23,746 & 27,418 & 36,993 & 0,264 \\
\hline & $h=5$ & $T^{*}$ & 7,110 & 9,676 & 12,636 & 14,571 & 19,324 & 0,020 \\
\hline & $\mathrm{j}=5$ & $\chi^{2}$ & 8,343 & 11,389 & 14,684 & 16,919 & 21,666 & \\
\hline & & $\hat{\hat{d}}_{S}$ & & & & & & 0,072 \\
\hline & & $\mathrm{T}$ & 21,239 & 27,636 & 34,695 & 39,251 & 47,044 & 0,182 \\
\hline & $h=10$ & $T^{*}$ & 12,188 & 14,551 & 17,997 & 19,623 & 22,916 & 0,000 \\
\hline & $\mathrm{j}=5$ & $\chi^{2}$ & 18,338 & 22,718 & 27,204 & 30,144 & 36,191 & \\
\hline & & $\hat{d}_{S}$ & & & & & & 0,110 \\
\hline
\end{tabular}

Tabela B.5: Influência do Coeficiente Autoregressivo $(\phi)$ do Processo ARMA com Distribuição Gaussiana no Teste MLC. 


\begin{tabular}{|c|c|c|c|c|c|c|c|c|}
\hline & & & \multicolumn{5}{|c|}{ Quantil } & \multirow{2}{*}{$\begin{array}{l}\text { Prop. } \\
\operatorname{rej}(5 \%)\end{array}$} \\
\hline & & & 0,5 & 0,75 & 0,90 & 0,95 & 0,99 & \\
\hline \multirow{12}{*}{$\theta=-0,6$} & & $\mathrm{~T}$ & 8,094 & 11,888 & 16,218 & 18,353 & 23,821 & 0,074 \\
\hline & $\mathrm{h}=5$ & $T^{*}$ & 6,696 & 9,231 & 12,471 & 14,082 & 17,650 & 0,016 \\
\hline & $\mathrm{j}=3$ & $\chi^{2}$ & 8,343 & 11,389 & 14,684 & 16,919 & 21,666 & \\
\hline & & $\hat{d}_{S}$ & & & & & & 0,102 \\
\hline & & $\mathrm{T}$ & 8,277 & 11,480 & 14,729 & 17,373 & 21,144 & 0,056 \\
\hline & $\mathrm{h}=5$ & $T^{*}$ & 6,523 & 8,800 & 11,169 & 13,112 & 16,810 & 0,010 \\
\hline & $\mathrm{j}=5$ & $\chi^{2}$ & 8,343 & 11,389 & 14,684 & 16,919 & 21,666 & \\
\hline & & & & & & & & 0,076 \\
\hline & & $\mathrm{T}$ & 17,705 & 22,459 & 27,191 & 31,002 & 37,674 & 0,064 \\
\hline & $h=10$ & $T^{*}$ & 13,333 & 16,679 & 19,510 & 21,940 & 27,027 & 0,002 \\
\hline & $\mathrm{j}=5$ & $\chi^{2}$ & 18,338 & 22,718 & 27,204 & 30,144 & 36,191 & \\
\hline & & $\hat{d}_{S}$ & & & & & & 0,066 \\
\hline \multirow{12}{*}{$\theta=-0,2$} & & $\overline{T T}$ & 8,209 & $\begin{array}{l}11,103 \\
\end{array}$ & $\overline{14,714}$ & $\bar{~} 16,847$ & $\bar{~} 19,841$ & 0,048 \\
\hline & $h=5$ & $T^{*}$ & 7,853 & 10,531 & 14,002 & 16,215 & 19,445 & 0,030 \\
\hline & $j=3$ & $\chi^{2}$ & 8,343 & 11,389 & 14,684 & 16,919 & 21,666 & \\
\hline & & $\hat{d}_{S}$ & & & & & & 0,080 \\
\hline & & $\mathrm{T}$ & 7,937 & 10,883 & 14,101 & 16,184 & 19,193 & 0,040 \\
\hline & $\mathrm{h}=5$ & $T^{*}$ & 7,792 & 10,555 & 13,500 & 15,093 & 18,624 & 0,016 \\
\hline & $\mathrm{j}=5$ & $\chi^{2}$ & 8,343 & 11,389 & 14,684 & 16,919 & 21,666 & \\
\hline & & $\hat{d}_{S}$ & & & & & & 0,068 \\
\hline & & $T$ & 17,795 & 21,603 & 25,920 & 29,081 & 34,053 & 0,040 \\
\hline & $h=10$ & $T^{*}$ & 16,892 & 20,670 & 24,985 & 27,015 & 32,075 & 0,016 \\
\hline & $\mathrm{j}=5$ & $\chi^{2}$ & 18,338 & 22,718 & 27,204 & 30,144 & 36,191 & \\
\hline & & $\hat{d}_{S}$ & & & & & & 0,076 \\
\hline \multirow{12}{*}{$\theta=0,2$} & & $\mathrm{~T}$ & 8,413 & 11,506 & $\overline{14,754}$ & 16,295 & 20,745 & 0,038 \\
\hline & $\mathrm{h}=5$ & $T^{*}$ & 7,882 & 10,694 & 13,573 & 14,998 & 18,915 & 0,020 \\
\hline & $j=3$ & $\chi^{2}$ & 8,343 & 11,389 & 14,684 & 16,919 & 21,666 & \\
\hline & & $\hat{d}_{S}$ & & & & & & 0,074 \\
\hline & & $T$ & 8,515 & $\begin{array}{ll}11,291 \\
\end{array}$ & 14,009 & 16,379 & 20,791 & 0,046 \\
\hline & $\mathrm{h}=5$ & $T^{*}$ & 7,959 & 10,642 & 13,312 & 15,138 & 19,630 & 0,028 \\
\hline & $\mathrm{j}=5$ & $\chi^{2}$ & 8,343 & 11,389 & 14,684 & 16,919 & 21,666 & \\
\hline & & $\hat{d}_{S}$ & & & & & & 0,074 \\
\hline & & $\mathrm{T}$ & 18,683 & 23,677 & 27,205 & 31,474 & 38,382 & 0,064 \\
\hline & $h=10$ & $T^{*}$ & 17,739 & 21,796 & 25,200 & 28,440 & 36,952 & 0,040 \\
\hline & $\mathrm{j}=5$ & $\chi^{2}$ & 18,338 & 22,718 & 27,204 & 30,144 & 36,191 & \\
\hline & & $\hat{d}_{S}$ & & & & & & 0,082 \\
\hline \multirow{12}{*}{$\theta=0,6$} & & $\bar{T}$ & 9,673 & $\overline{c 12,834}$ & $=16,822$ & $\overline{19,032}$ & 24,628 & 0,094 \\
\hline & $h=5$ & $T^{*}$ & 7,296 & 9,884 & 12,453 & 14,112 & 17,991 & 0,014 \\
\hline & $\mathrm{j}=3$ & $\chi^{2}$ & 8,343 & 11,389 & 14,684 & 16,919 & 21,666 & \\
\hline & & $\hat{d}_{S}$ & & & & & & 0,092 \\
\hline & & $\mathrm{T}$ & 8,911 & 12,534 & 15,555 & 18,268 & 24,999 & 0,082 \\
\hline & $\mathrm{h}=5$ & $T^{*}$ & 6,795 & 9,402 & 12,005 & 14,069 & 19,407 & 0,018 \\
\hline & $\mathrm{j}=5$ & $\chi^{2}$ & 8,343 & 11,389 & 14,684 & 16,919 & 21,666 & \\
\hline & & $\hat{d}_{S}$ & & & & & & 0,120 \\
\hline & & $\mathrm{T}$ & 18,900 & 23,704 & 28,598 & 31,758 & 39,353 & 0,078 \\
\hline & $h=10$ & $T^{*}$ & 14,206 & 17,679 & 21,148 & 23,292 & 27,147 & 0,004 \\
\hline & $\mathrm{j}=5$ & $\chi^{2}$ & 18,338 & 22,718 & 27,204 & 30,144 & 36,191 & \\
\hline & & $\hat{d}_{S}$ & & & & & & 0,114 \\
\hline
\end{tabular}

Tabela B.6: Influência do Coeficiente de Médias Móveis $(\theta)$ do Processo ARMA com Distribuição Gaussiana no Teste MLC. 


\begin{tabular}{|c|c|c|c|c|c|c|c|c|}
\hline & & & \multicolumn{5}{|c|}{ Quantil } & \multirow{2}{*}{$\begin{array}{l}\text { Prop. } \\
\text { rej(5\%) }\end{array}$} \\
\hline & & & 0,5 & 0,75 & 0,90 & 0,95 & 0,99 & \\
\hline \multirow{12}{*}{$\phi=-0,6$} & & $\mathrm{~T}$ & 50,911 & 63,017 & 74.194 & 81,370 & 93,061 & 0,998 \\
\hline & $\mathrm{h}=5$ & $T^{*}$ & 29,389 & 34,754 & 41,392 & 48,378 & 52,913 & 0,956 \\
\hline & $\mathrm{j}=3$ & $\chi^{2}$ & 8,343 & 11,389 & 14,684 & 16,919 & 21,666 & \\
\hline & & $\hat{\hat{d}}_{S}$ & & & & & & 0,302 \\
\hline & & $\mathrm{T}$ & 15,105 & 22,748 & 30,025 & 35,892 & 45,155 & 0,442 \\
\hline & $\mathrm{h}=5$ & $T^{*}$ & 8,098 & 11,487 & 15,192 & 16,589 & 20,097 & 0,046 \\
\hline & $\mathrm{j}=5$ & $\chi^{2}$ & 8,343 & 11,389 & 14,684 & 16,919 & 21,666 & \\
\hline & & & & & & & & 0,120 \\
\hline & & $\mathrm{T}$ & 23,049 & 30,550 & 39,965 & 45,933 & 56,290 & 0,262 \\
\hline & $h=10$ & $T^{*}$ & 12,366 & 15,646 & 19,055 & 21,753 & 27,316 & 0,004 \\
\hline & $\mathrm{j}=5$ & $\chi^{2}$ & 18,338 & 22,718 & 27,204 & 30,144 & 36,191 & \\
\hline & & $\hat{d}_{S}$ & & & & & & 0,122 \\
\hline \multirow{12}{*}{$\phi=-0,2$} & & $\overline{\mathrm{T}}$ & 8,517 & $\overline{111,876}$ & $\overline{15,784}$ & $\overline{18,192}$ & $\overline{23,781}$ & $\overline{0,078}$ \\
\hline & $\mathrm{h}=5$ & $T^{*}$ & 7,948 & 11,118 & 14,253 & 16,743 & 19,986 & 0,050 \\
\hline & $j=3$ & $\chi^{2}$ & 8,343 & 11,389 & 14,684 & 16,919 & 21,666 & \\
\hline & & $\hat{d}_{S}$ & & & & & & 0,096 \\
\hline & & $T$ & 8,644 & 11,983 & 15,175 & 18,063 & 22,881 & 0,070 \\
\hline & $\mathrm{h}=5$ & $T^{*}$ & 7,929 & 10,778 & 14,256 & 16,475 & 21,740 & 0,046 \\
\hline & $\mathrm{j}=5$ & $\chi^{2}$ & 8,343 & 11,389 & 14,684 & 16,919 & 21,666 & \\
\hline & & $\hat{\hat{d}}_{S}$ & & & & & & 0,082 \\
\hline & & $T$ & 18,130 & 22,322 & 27,767 & 31,017 & 36,080 & 0,060 \\
\hline & $h=10$ & $T^{*}$ & 16,708 & 20,887 & 24,966 & 27,734 & 31,938 & 0,022 \\
\hline & $\mathrm{j}=5$ & $\chi^{2}$ & 18,338 & 22,718 & 27,204 & 30,144 & 36,191 & \\
\hline & & $\hat{\hat{d}}_{S}$ & & & & & & 0,074 \\
\hline \multirow{12}{*}{$\phi=0,2$} & & $\bar{T}$ & 7,985 & $\overline{111,004}$ & 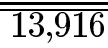 & $\overline{10,032}$ & 20,877 & $\overline{00,036}$ \\
\hline & $h=5$ & $T^{*}$ & 7.798 & 10,364 & 13.734 & 15,395 & 20,332 & 0.026 \\
\hline & $j=3$ & $\chi^{2}$ & 8,343 & 11,389 & 14,684 & 16,919 & 21,666 & \\
\hline & & $\hat{d}_{S}$ & & & & & & 0,092 \\
\hline & & $\stackrel{s}{T}$ & 7,922 & 11,055 & 14,160 & 17,224 & 19,964 & 0,054 \\
\hline & $\mathrm{h}=5$ & $T^{*}$ & 7,784 & 10,430 & 13,399 & 15,837 & 18,710 & 0,030 \\
\hline & $\mathrm{j}=5$ & $\chi^{2}$ & 8,343 & 11,389 & 14,684 & 16,919 & 21,666 & \\
\hline & & $\hat{\hat{d}}_{S}$ & & & & & & 0,106 \\
\hline & & $T$ & 17,903 & 21,870 & 25,797 & 28,943 & 36,819 & 0,044 \\
\hline & $h=10$ & $T^{*}$ & 16,776 & 20,513 & 24,295 & 26,549 & 33,269 & 0,020 \\
\hline & $\mathrm{j}=5$ & $\chi^{2}$ & 18,338 & 22,718 & 27,204 & 30,144 & 36,191 & \\
\hline & & $\hat{d}_{S}$ & & & & & & 0,062 \\
\hline \multirow{12}{*}{$\phi=0,6$} & & $\bar{T}$ & 48,806 & 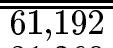 & 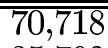 & 77,457 & $\overline{990,391}$ & $\overline{1} 1,000$ \\
\hline & $h=5$ & $T^{*}$ & 26,150 & 31,260 & 35,792 & 37,459 & 42,214 & 0,908 \\
\hline & $j=3$ & $\chi^{2}$ & 8,343 & 11,389 & 14,684 & 16,919 & 21,666 & \\
\hline & & $\hat{d}_{S}$ & & & & & & 0,100 \\
\hline & & $T$ & 11,176 & 16,213 & 23,260 & 27,822 & 34,522 & 0,228 \\
\hline & $\mathrm{h}=5$ & $T^{*}$ & 6,745 & 9,617 & 12,642 & 14,153 & 18,447 & 0,016 \\
\hline & $\mathrm{j}=5$ & $\chi^{2}$ & 8,343 & 11,389 & 14,684 & 16,919 & 21,666 & \\
\hline & & $\hat{\hat{d}}_{S}$ & & & & & & 0,104 \\
\hline & & $\mathrm{T}$ & 20,866 & 27,837 & 36,357 & 42,738 & 51,899 & 0,196 \\
\hline & $\mathrm{h}=10$ & $T^{*}$ & 11,870 & 14,981 & 18,096 & 20,385 & 23,449 & 0,002 \\
\hline & $\mathrm{j}=5$ & $\chi^{2}$ & 18,338 & 22,718 & 27,204 & 30,144 & 36,191 & \\
\hline & & $\hat{d}_{S}$ & & & & & & 0,086 \\
\hline
\end{tabular}

Tabela B.7: Influência do Coeficiente Autoregressivo $(\phi)$ do Processo ARMA com Distribuição Qui-quadrado no Teste MLC. 


\begin{tabular}{|c|c|c|c|c|c|c|c|c|}
\hline & & & \multicolumn{5}{|c|}{ Quantil } & \multirow{2}{*}{$\begin{array}{l}\text { Prop. } \\
\text { rej(5\%) }\end{array}$} \\
\hline & & & $\overline{0,5}$ & 0,75 & 0,90 & 0,95 & 0,99 & \\
\hline \multirow{12}{*}{$\theta=-0,6$} & & $\mathrm{~T}$ & 8,325 & 12,222 & 15,604 & 17,323 & 21,772 & 0,066 \\
\hline & $\mathrm{h}=5$ & $T^{*}$ & 6,908 & 9,217 & 12,143 & 13,453 & 17,494 & 0,012 \\
\hline & $\mathrm{j}=3$ & $\chi^{2}$ & 8,343 & 11,389 & 14,684 & 16,919 & 21,666 & \\
\hline & & $\hat{d}_{S}$ & & & & & & 0,094 \\
\hline & & $\mathrm{T}$ & 8,408 & 11,629 & 15,817 & 17,436 & 22,582 & 0,064 \\
\hline & $\mathrm{h}=5$ & $T^{*}$ & 6,664 & 9,044 & 11,865 & 14,033 & 17,106 & 0,012 \\
\hline & $\mathrm{j}=5$ & $\chi^{2}$ & 8,343 & 11,389 & 14,684 & 16,919 & 21,666 & \\
\hline & & & & & & & & 0,082 \\
\hline & & $\mathrm{T}$ & 16,724 & 21,932 & 27,810 & 31,721 & 37,249 & 0,070 \\
\hline & $\mathrm{h}=10$ & $T^{*}$ & 13,004 & 16,925 & 20,252 & 22,316 & 25,425 & 0,004 \\
\hline & $\mathrm{j}=5$ & $\chi^{2}$ & 18,338 & 22,718 & 27,204 & 30,144 & 36,191 & \\
\hline & & $\hat{\hat{d}}_{S}$ & & & & & & 0,088 \\
\hline \multirow{12}{*}{$\theta=-0,2$} & & $\mathrm{~T}$ & $\overline{77,732}$ & $\overline{10,757}$ & $\overline{14,298}$ & $\overline{17,226}$ & 23,038 & 0,054 \\
\hline & $\mathrm{h}=5$ & $T^{*}$ & 7,549 & 10,403 & 13,648 & 16,076 & 20,386 & 0,042 \\
\hline & $j=3$ & $\chi^{2}$ & 8,343 & 11,389 & 14,684 & 16,919 & 21,666 & \\
\hline & & $\hat{\hat{d}}_{S}$ & & & & & & 0,078 \\
\hline & & $T$ & 7,738 & 11,358 & 14,761 & 17,043 & 21,271 & 0,052 \\
\hline & $\mathrm{h}=5$ & $T^{*}$ & 7,587 & 10,601 & 13,531 & 15,936 & 21,472 & 0,038 \\
\hline & $\mathrm{j}=5$ & $\chi^{2}$ & 8,343 & 11,389 & 14,684 & 16,919 & 21,666 & \\
\hline & & $\hat{\hat{d}}_{S}$ & & & & & & 0,088 \\
\hline & & $T$ & 17,201 & 22,159 & 27,271 & 30,046 & 35,242 & 0,048 \\
\hline & $h=10$ & $T^{*}$ & 16,515 & 20,846 & 25,013 & 28,428 & 34,157 & 0,030 \\
\hline & $\mathrm{j}=5$ & $\chi^{2}$ & 18,338 & 22,718 & 27,204 & 30,144 & 36,191 & \\
\hline & & $\hat{\hat{d}}_{S}$ & & & & & & 0,092 \\
\hline \multirow{12}{*}{$\theta=0,2$} & & $\mathrm{~T}$ & $\overline{8,088}$ & $\overline{111,265}$ & $\overline{14,684}$ & $\overline{17,072}$ & $\overline{222,860}$ & 0,054 \\
\hline & $\mathrm{h}=5$ & $T^{*}$ & 7,570 & 10,687 & 13,920 & 15,207 & 19,546 & 0,034 \\
\hline & $j=3$ & $\chi^{2}$ & 8,343 & 11,389 & 14,684 & 16,919 & 21,666 & \\
\hline & & $\hat{d}_{S}$ & & & & & & 0,078 \\
\hline & & $T$ & 8,356 & 11,797 & 15,357 & 18,729 & 25,204 & 0,080 \\
\hline & $\mathrm{h}=5$ & $T^{*}$ & 7,937 & 10,948 & 14,639 & 16,936 & 23,720 & 0,054 \\
\hline & $\mathrm{j}=5$ & $\chi^{2}$ & 8,343 & 11,389 & 14,684 & 16,919 & 21,666 & \\
\hline & & $\hat{d}_{S}$ & & & & & & 0,082 \\
\hline & & $T$ & 18,011 & 23,193 & 28,192 & 30,402 & 37,733 & 0,054 \\
\hline & $h=10$ & $T^{*}$ & 17,069 & 21,749 & 25,597 & 27,746 & 33,758 & 0,024 \\
\hline & $\mathrm{j}=5$ & $\chi^{2}$ & 18,338 & 22,718 & 27,204 & 30,144 & 36,191 & \\
\hline & & $\hat{d}_{S}$ & & & & & & 0,110 \\
\hline \multirow{12}{*}{$\theta=0,6$} & & $\mathrm{~T}$ & $\overline{99,306}$ & $\overline{12,925}$ & $\overline{16,787}$ & $\overline{18,725}$ & $\overline{27,978}$ & 0,094 \\
\hline & $\mathrm{h}=5$ & $T^{*}$ & 6,999 & 9,564 & 11,872 & 13,386 & 19,812 & 0,018 \\
\hline & $j=3$ & $\chi^{2}$ & 8,343 & 11,389 & 14,684 & 16,919 & 21,666 & \\
\hline & & $\hat{\hat{d}}_{S}$ & & & & & & 0,116 \\
\hline & & $T$ & 8,923 & 13,092 & 16,486 & 18,426 & 25,171 & 0,086 \\
\hline & $\mathrm{h}=5$ & $T^{*}$ & 7,062 & 9,823 & 12,132 & 14,065 & 17,707 & 0,014 \\
\hline & $\mathrm{j}=5$ & $\chi^{2}$ & 8,343 & 11,389 & 14,684 & 16,919 & 21,666 & \\
\hline & & $\hat{\hat{d}}_{S}$ & & & & & & 0,100 \\
\hline & & $T$ & 18,993 & 24,050 & 30,077 & 33,883 & 40,613 & 0,098 \\
\hline & $\mathrm{h}=10$ & $T^{*}$ & 14,241 & 17,989 & 22,096 & 25,396 & 31,684 & 0,016 \\
\hline & $\mathrm{j}=5$ & $\chi^{2}$ & 18,338 & 22,718 & 27,204 & 30,144 & 36,191 & \\
\hline & & $\hat{\hat{d}}_{S}$ & & & & & & 0,118 \\
\hline
\end{tabular}

Tabela B.8: Influência do Coeficiente de Médias Móveis $(\theta)$ do Processo ARMA com Distribuição Qui-quadrado no Teste MLC. 


\section{Referências}

Anderson, T.W. (1971). The Statistical Analysis of Time Series. New York: Wiley.

Baillie, R.T. (1996). Long memory processes and fractional integration in econometrics. Journal of Econometrics, 73, 5-59.

Box, G.E.P and Jenkins, G.M. and Reinsel, G. (1994). Time Series analysis: Forecasting and Control. Third Edition. Englewood Cliffs: Prentice Hall.

Box, G.E.P. and Tiao, G.C. (1977). A canonical analysis of multiple time series. Biometrika, 64, 355-365.

Breidt, F.J., Crato, N. and de Lima, P. (1998). On the detection and estimation of long memory in stochastic volatility. Journal of Econometrics, 83, 35-348.

Brillinger, D.R. (1981). Time Series: Data Analysis and Theory. 2nd ed. San Francisco: Holden-Day.

Chen, G., Abraham, B., and Peiris, S. (1993). Lag window estimation of the degree of differencing in fractionally integrated time series models. Journal of Time Series Analysis, 15, 473-487.

Cheung, Y.W. and Lai, K.S. (1993). A fractional cointegration analysis of purchasing 
power parity. Journal of Business and Economics Statistics, 11, 103-112.

Diebolt, C. and Guiraud, V. (2002). A note on long memory time series. Unpublished Working Paper. Université Montpellier.

Ding, Z., Granger, C.W.J. and Engle, R.F. (1993). A long memory property of stocks returns and a new model. Journal of Empirical Finance, 1, 83-106.

Engle, R.F. (1982). Autoregressive conditional heteroskedasticity with estimates of the variance of U.K. inflation. Econometrica, 50, 987-1008.

Engle, R.F. and Granger, C.W.J. (1987). Co-integration and error correction: representation, estimating and testing. Econometrica, 55, 251-276.

Fuller, W.A. (1996). Introduction to Statistical Time Series. New York: Wiley.

Geweke, J. and Porter-Hudak, S. (1983). The estimation and application of long memory time series models. Journal of Time Series Analysis, 4, 221-238.

Glosten, L.R., Jagannathan, R. and Runkle, D. (1993). Relationship between the expected value and the volatility of the nominal excess return on stocks. Journal of Finance, 48, 1779-1801.

Granger, C.W.J. (1980). Long memory relationships and the aggregation of dynamic models. Journal of Econometrics, 14, 227-238.

Granger, C.W.J. and Joyeuz, R. (1980). An introduction to long memory time series models and fractional differencing. Journal of Time Series Analysis, 1, 15-39.

Harvey, A.C. (1993). Long memory in stochastic volatility. Unpublished working paper, London School of Economics, London, UK.

Hipel, K.W. and McLeod, A.I. (1978a). Preservation of the rescales ajusted range, 2: 
Simulation Studies Using Box-Jenkins Models, Water resource research, 14, 506-516.

Hipel, K.W. and McLeod, A.I. (1978b). Preservation of the rescales ajusted range, 3: Fractional Gaussian noise algorithms, Water Resource Research, 14, 517-518.

Hosking, J.R.M. (1981). Fractional differencing. Biometrika, 68, 165-176.

Hotteling, H. (1936). Relations between two sets of variates. Biometrika, 28, 321-377.

Hurst, H.E. (1951). Long-term storage capacity of reserviors. Transaction of the American Society of Civil Engineers, 116, 770-799.

Hurst, H.E. (1956). Methods of using long term storage in reservoirs. Proceedings of the Institute of Civil Engineers, 1, 519-543.

Hurst, H.E. (1957). A suggested statiscal model of some time series that occur in nature. Nature, 180, 494.

Johansen, S. (1991). Estimation and hypothesis testing of co-integration vectors in gaussian vector regression models. Econometrica, 59, 1551-1580.

Lawrance, A.J. and Kottegoda, N.T. (1977). Sochastic modelling of riverflow time series. Journal of the Royal Statistical Society A, 140, 1-47.

Lo, A.W. (1991). Long term memory in stock market prices. Econometrica, 59, 12791313.

McLeod, A.I. and Hipel, K.W. (1978). Preservation of the rescaled adjusted range, 1: A reassessment of the Hurst phenomenon. Water Resources Research, 14, 491-508.

Morettin, P.A. e Toloi, C.M.C. (1981). Modelos para Previsão de Séries Temporais. Rio de Janeiro. IMPA. 
Moura, C.B. (1997). Comparação de estimadores do parâmetro de memória longa do modelo ARFIMA $(p, d, q)$. Dissertação de Mestrado (IME-USP, São Paulo).

Nelson, D.B. (1991). Conditional heteroskedasticity in asset returns. Econometrica, 59, 347-370.

Ray, B.K. and Tsay, R.S. (1997). Identifying common long-range dependence in a vector time series. Working paper, University of Chicago, Statistics Research Center, Graduate School of Business.

Reisen, V.A. (1994). Estimation of the fractional difference parameter in the ARIMA(p,d,q) model using the smoothed periodogram. Journal of Time Series Analysis, 15, 335-351.

Robinson, P.M. (1995). Gaussian semiparametric estimation of long range dependence. Annals of Statistics, 23, 1630-1661.

Robinson, P.M. and Henry,M. (1999). Long and short memory conditional heteroskedasticity in estimating the memory parameters os levels. Econometric Theory, 15, 229-336.

Shumway, R.H. and Stoffer, D.S. (2000). Time Series Analysis and its Applications. New York. Springer.

Shuster, A. (1906). On the periodicities of sunspots. Phil. Trans. R. Soc. Ser. A, 206, 69-100.

Sowell, F.B. (1986). Fractionally integrated vector time series. Ph.D. dissertation (Duke University, Durham, NC).

Sowell, F.B. (1992). Maximum likilihood estimation of stationary univariate fractionally integrated time series models. Journal of Econometrics, 53, 165-188.

Stock, J.H. and Watson, M.W. (1988). Testing for common trends. Journal of American Statistician Society, 83, 1097-1107. 
Taylor, S.J. (1980). Conjectured models for trend in financial prices tests as forecasts. Journal of the Royal Statistical Society, Series B, 42, 338-362.

Taylor, S.J. (1986). Modelling Financial Time Series. New York: Wiley.

Tiao, G.C. and Tsay, R.S. (1989). Model specification in multivariate time series. Journal of Royal Statistical Society B, 51, 157-213.

Tsay, R.S. (1989). Identifying multivariate time series models. Journal of Time Series Analysis, 10, 357-372.

Tsay, R.S. and Tiao, G.C. (1985). Use of canonical analysis in time series identification. Biometrika, 72, 299-316.

Velasco, C. (1999). Gaussian semiparametric estimation of non-stationary time series. Journal of Time Series Analysis, 20, 87-127. 